\title{
International workshop on next generation gamma-ray source
}

\author{
C R Howell ${ }^{1,2}$, M W Ahmed ${ }^{2,3, *}\left(\mathbb{D}\right.$, A Afanasev $^{4} \odot$, D Alesini ${ }^{5}$, \\ J R M Annand ${ }^{6}$, A Aprahamian ${ }^{7}$, D L Balabanski ${ }^{8}$, \\ S V Benson ${ }^{9}$, A Bernstein ${ }^{10}$, C R Brune ${ }^{11}$, J Byrd ${ }^{12}$, \\ B E Carlsten ${ }^{13}$, A E Champagne ${ }^{2,14}$, S Chattopadhyay ${ }^{15}$, \\ D Davis',16, E J Downie ${ }^{4}$, J M Durham ${ }^{13}$, G Feldman ${ }^{4}$, \\ H Gao ${ }^{1,2}$, C G R Geddes ${ }^{17}$, H W Grießhammer ${ }^{4}$, \\ R Hajima ${ }^{18}\left(\mathbb{D}, \mathbf{H ~ H a o}^{1,2}\right.$, D Hornidge ${ }^{19}$, J Isaak ${ }^{20}$, \\ R V F Janssens ${ }^{2,14}$, D P Kendellen ${ }^{1,2}$, M A Kovash ${ }^{21}$, \\ P P Martel ${ }^{22}$, U-G Meißner ${ }^{23}{ }^{\circ}$, R Miskimen ${ }^{24}$, B Pasquini ${ }^{25}$, \\ D R Phillips ${ }^{11}{ }^{1}$, N Pietralla ${ }^{20}$, D Savran ${ }^{26}$, M R Schindler ${ }^{27}$, \\ M H Sikora ${ }^{2,4}$, W M Snow ${ }^{28}$ (D, R P Springer ${ }^{1}$, C Sun ${ }^{17}$, \\ C Tang ${ }^{29}$, B Tiburzi ${ }^{30}$ (D) A P Tonchev ${ }^{31}$, W Tornow ${ }^{3,18}$, \\ C A Ur ${ }^{8}$, D Wang ${ }^{32}$, H R Weller ${ }^{1,2}$, V Werner ${ }^{20}$, Y K Wu $^{1,2}$, \\ J Yan ${ }^{1,2}$, Z Zhao ${ }^{33}$, A Zilges ${ }^{34}$ and F Zomer ${ }^{35}$ \\ ${ }^{1}$ Department of Physics, Duke University, Durham, NC 27708, United States of \\ America \\ 2 Triangle Universities Nuclear Laboratory, Durham, NC 27708, United States of \\ America \\ ${ }^{3}$ Department of Mathematics and Physics, North Carolina Central University, \\ Durham, NC 27707, United States of America \\ ${ }^{4}$ Department of Physics, The George Washington University, Washington, D.C. \\ 20052, United States of America \\ 5 LNF-INFN, Via E Fermi 40, 00044 Frascati (Rome), Italy \\ ${ }^{6}$ SUPA School of Physics and Astronomy, University of Glasgow, Glasgow, G12 \\ 8QQ, United Kingdom \\ 7 Department of Physics, University of Notre Dame, Notre Dame, IN 46556, United \\ States of America \\ ${ }^{8}$ Extreme Light Infrastructure Nuclear Physics (ELI-NP), Horia Hulubei National \\ Institute for R \& D in Physics and Nuclear Engineering (IFIN-HH), Magurele, \\ Romania \\ 9 Thomas Jefferson National Accelerator Facility, Newport News, VA 23606, United \\ States of America \\ 10 Physics Department and Laboratory for Nuclear Science Massachusetts Institute \\ of Technology, Cambridge, MA 02139, United States of America
}

*Author to whom any correspondence should be addressed.

Original content from this work may be used under the terms of the Creative Commons Attribution 4.0 licence. Any further distribution of this work must maintain attribution to the author(s) and the title of the work, journal citation and DOI. 
11 Department of Physics and Astronomy, Ohio University, Athens, OH 45701, United States of America

12 Argonne National Laboratory, Argonne, Illinois 60439, United States of America

${ }^{13}$ Los Alamos National Laboratory, Los Alamos, NM 87545, United States of America

${ }^{14}$ Department of Physics and Astronomy, University of North Carolina, Chapel Hill, NC, United States of America

15 Fermi National Accelerator Laboratory, Batavia, IL 60510, United States of America

${ }^{16}$ Department of Physics, North Carolina State University, Raleigh, NC 27695, United States of America

${ }^{17}$ Lawrence Berkeley National Laboratory, Berkeley, CA 94720, United States of America

18 National Institutes for Quantum and Radiological Science and Technology, Tokai, Naka, Ibaraki 3191106, Japan

${ }_{19}$ Mount Allison University, Sackville, New Brunswick E4L1E6, Canada

${ }^{20}$ Institut für Kernphysik, Technische Universität Darmstadt, 64289 Darmstadt, Germany

${ }^{21}$ Department of Physics and Astronomy, University of Kentucky, Lexington, KY 40506, United States of America

${ }^{22}$ Institut für Kernphysik, University of Mainz, D-55099 Mainz, Germany

${ }^{23}$ Helmholtz-Institut für Strahlen-und Kernphysik and Bethe Center for Theoretical Physics, Universität Bonn, D-53115 Bonn, Germany

${ }^{24}$ University of Massachusetts, Amherst, MA 01003, United States of America

25 University of Pavia, Pavia, Italy

${ }^{26}$ GSI Helmholtzzentrum für Schwerionenforschung GmbH, 64291 Darmstadt, Germany

27 Department of Physics and Astronomy, University of South Carolina, Columbia, SC, United States of America

${ }^{28}$ Department of Physics, Indiana University, Bloomington, IN 47408, United States of America

29 Department of Engineering Physics, Tsinghua University, Beijing 100084, People's Republic of China

30 City College of New York, New York, NY, United States of America

31 Nuclear and Chemical Sciences Division, Lawrence Livermore National

Laboratory, Livermore, CA 94550, United States of America

32 Shanghai Institute of Applied Physics, Chinese Academy of Sciences, Shanghai 201800, People's Republic of China

${ }^{33}$ University of Virginia, Charlottesville, VA, United States of America

${ }^{34}$ Institut für Kernphysik, Universität zu Köln, Zülpicher Straße 77, D-50937 Köln, Germany

${ }^{35}$ LAL, Université Paris-Sud, CNRS/IN2P3, Orsay, France

E-mail: mahmed2@nccu.edu

Received 13 May 2021, revised 11 June 2021

Accepted for publication 20 September 2021

Published 2 December 2021

\section{Abstract}

A workshop on The Next Generation Gamma-Ray Source sponsored by the Office of Nuclear Physics at the Department of Energy, was held November 17-19, 2016 in Bethesda, Maryland. The goals of the workshop were to 
identify basic and applied research opportunities at the frontiers of nuclear physics that would be made possible by the beam capabilities of an advanced laser Compton beam facility. To anchor the scientific vision to realistically achievable beam specifications using proven technologies, the workshop brought together experts in the fields of electron accelerators, lasers, and optics to examine the technical options for achieving the beam specifications required by the most compelling parts of the proposed research programs. An international assembly of participants included current and prospective $\gamma$-ray beam users, accelerator and light-source physicists, and federal agency program managers. Sessions were organized to foster interactions between the beam users and facility developers, allowing for information sharing and mutual feedback between the two groups. The workshop findings and recommendations are summarized in this whitepaper.

Keywords: gamma-ray, Compton scattering, nuclear astrophysics, nuclear structure, hadronic parity violation, low-energy QCD, nuclear theory

(Some figures may appear in colour only in the online journal)

\section{Executive summary}

The photon is a theoretically well-understood probe for investigating the structure of matter over a wide range of distance and energy scales. The angular-momentum selectivity and unconstrained transfer of isospin in most gamma-ray ( $\gamma$-ray) induced reactions enable highly precise strategic investigations of nuclear and nucleon structure and of collective motion responses of the internal degrees of freedom associated with electric charge and current distributions. In addition, high-energy photons are well suited for non-intrusive material analysis applications in areas such as homeland security, nuclear security, structural integrity assessments, and medical diagnostics.

Bremsstrahlung radiation produced by an electron beam incident on a high- $Z$ target has been the workhorse photon-beam source used in nuclear physics research and applications for over a century. The energy spectrum of a bremsstrahlung $\gamma$-ray beam enables measurements of nuclear structure and reaction dynamics over a continuous energy range in a single experiment. Tagged bremsstrahlung sources provide the capability of associating the energy of the photon inducing a reaction with the photons detected in the final state. Also, coherent bremsstrahlung beams produced using a crystal target offer researchers a partially polarized $\gamma$-ray beam at energies near the end point of the bremsstrahlung spectrum. The scientific insight provided by research conducted using these sources is impressive, and facilities with advanced bremsstrahlung photon sources continue to be used in highly productive research programs. However, the continuous energy nature of bremsstrahlung $\gamma$-ray beams, which facilitates survey measurements over a broad energy range, also has the adverse effect of creating backgrounds that limit the sensitivity of the experiments. These backgrounds make it difficult to investigate nuclear phenomena with extremely low cross sections or to perform measurements on targets with small sample sizes, as is the case for isotopes with low natural abundances.

The nearly monoenergetic $\gamma$-ray beams produced by laser Compton scattering offer an alternative to bremsstrahlung beams, providing an enhanced signal-to-background ratio in basic and applied research, and reducing the radiation exposure in material analysis applications. In addition, laser Compton $\gamma$-ray beams can be produced with a beam polarization greater than 
95\% for both linear and circular polarization. Linearly polarized laser Compton beams enable unambiguous determinations of the spin and parity of excited nuclear states, and beams with both linear and circular polarization are used in studies of the spin structure of nucleons.

Over the last three decades, laser Compton $\gamma$-ray beam facilities have provided intense polarized and nearly mono-energetic $\gamma$-ray beams for research programs in basic and applied nuclear physics. These facilities include GRAAL at the European Synchrotron Radiation Facility in Grenoble, LEGS at Brookhaven National Laboratory, LEPS at the SPring-8 facility, NewSUBARU at the University of Hyogo, and $\mathrm{HI} \gamma \mathrm{S}$ at the Triangle Universities Nuclear Laboratory. However, HI $\gamma \mathrm{S}$ and LEPS are the only facilities worldwide that are operated with nuclear physics as the primary research focus. These facilities will soon be joined by two more that are currently under construction, ELI-NP in Romania and the $\gamma$-ray beam line at the Shanghai Synchrotron Radiation Facility (SSRF) in China. These facilities will produce $\gamma$-ray beams with energies below $12 \mathrm{MeV}$ (ELI-NP), and below $20 \mathrm{MeV}$ and above $300 \mathrm{MeV}$ (SSRF).

Given that the time span between initial planning and the end of construction of an accelerator-driven light source is about a decade, consideration for next-generation laser Compton $\gamma$-ray sources should start now. Technological advances in electron accelerators, lasers, and optics made during the last decade create new options for producing intense polarized $\gamma$-ray beams with narrow energy widths at beam energies from around $1 \mathrm{MeV}$ to several $\mathrm{GeV}$, the energies relevant to nuclear-physics research. A workshop on The Next Generation GammaRay Sources, sponsored by the Office of Nuclear Physics at the Department of Energy, was held November 17-19, 2016 in Bethesda, Maryland. The goals of the workshop were to identify basic and applied research opportunities at the frontiers of nuclear physics that would be made possible by the beam capabilities of an advanced laser Compton beam facility. To anchor the scientific vision to realistically achievable beam specifications using proven technologies, the workshop brought together experts in the fields of electron accelerators, lasers, and optics to examine the technical options for achieving the beam specifications required by the most compelling parts of the proposed research programs. An international assembly of participants included current and prospective $\gamma$-ray beam users, accelerator and light-source physicists, and federal agency program managers. Sessions were organized to foster interactions between the beam users and facility developers, allowing for information sharing and mutual feedback between the two groups. The workshop findings and recommendations are summarized below.

\subsection{Findings of topical working groups}

The topical sessions at the workshop focused on five research areas: low-energy quantum chromodynamics (QCD), nuclear structure, nuclear astrophysics, fundamental symmetries, and applications. The advanced accelerator and light source technologies working group recommended laser Compton scattering inside an optical cavity as the primary technology for the next generation $\gamma$-ray sources. The working group concluded that two $\gamma$-ray beam facilities are needed to meet the beam requirements of the research presented in the topical sessions at the workshop (1) a medium-energy source with $\gamma$-ray beams in the range of 25 and $400 \mathrm{MeV}$, and (2) a low-energy source capable of delivering $\gamma$-ray beams from 1.5 to about $30 \mathrm{MeV}$. The topical sessions at the workshop focused on five main subjects: nucleon structure and low-energy QCD, nuclear structure, nuclear astrophysics, fundamental symmetries, and applications. Probing nucleon structure requires medium-energy photons, i.e., $\gamma$-ray beams with energies from about 60 to $350 \mathrm{MeV}$, while the four other research topics would use $\gamma$-ray beams at energies below about $20 \mathrm{MeV}$. Descriptions of the research opportunities for all five are in the science sections of this document. Summaries of the findings of the topical working groups are given below. 
1.1.1. Medium-energy NGLCGS facility. Understanding the emergence of hadron structure and the nuclear force in terms of QCD are key questions at the frontier of nuclear physics. These phenomena are a consequence of quarks and gluons interacting at confinement-scale distances, where color forces are strong. The beams at a medium-energy NGLCGS facility will enable measurements that uniquely probe hadron structure and hadronic interactions in this non-perturbative regime of QCD. The experimental program will investigate LEQCD phenomena with unprecedented precision in the photon energy range from about $60 \mathrm{MeV}$ to the nucleon-to-delta(1232) transition. The initial research program at such a facility will two main components: (1) Compton scattering to investigate nucleon structure and (2) photopion production to investigate the QCD origins of isospin symmetry breaking in the strong nuclear force. Both program components will require high-density polarized targets.

- Low-energy QCD and nucleon structure: the beam capabilities at a medium-energy NGLCGS will enable high-precision measurements of the scalar and spin nucleon polarizabilities by Compton scattering from unpolarized and polarized targets. Such measurements, together with advances in calculations using lattice QCD (LQCD) and QCD-based effective field theories, will explore the QCD origin of nucleon structure associated with the collective response of nucleons to electromagnetic impulses with unprecedented sensitivity. For example, the experimental programs at the NGLCGS facility are proposed to improve the statistical accuracy of the nucleon spin polarizability measurements by more than a factor of 10 from current values and to impact the dynamical scaler polarizabilities of the neutron and proton with precision from energies below to above the pion production threshold.

- Low-energy QCD: photopion production: the high intensity and high energy resolution of beams at a medium-energy NGLCGS facility will enable determination of electromagnetic $\mathrm{s}$-wave and p-wave amplitudes to the $\pi^{0}$ production with sufficiently high precision to observe charge-symmetry violation. Photoproduction of pions at energies near the reaction threshold provides mechanisms for investigation of the QCD origin of breaking of isospin symmetry in strong nuclear interactions.

1.1.2. Low-energy NGLCGS facility. The beams at low-energy NGLCGS facilities will enable high-accuracy measurements in nuclear structure, nuclear astrophysics, fundamental symmetries, and $\gamma$-ray beam applications in nuclear and homeland security. The research opportunities in each area are summarized below:

- Nuclear structure: the beams at an advanced $\gamma$-ray source will enable systematic studies of weak collective dipole and quadrupole nuclear excitations with unprecedented precision. Such studies will provide nuclear structure details and information about the symmetry energy of the nuclear equation of state (EOS) that are difficult to obtain by other means. The high $\gamma$-ray beam intensities will enable mapping of states accessed through $M 1$ transitions from the ground state in nuclei with a level of detail and breadth that will contribute to modeling of coherent neutrino-nucleus scattering and to calculating nuclear matrix elements for neutrinoless double-beta decay. Also, a next-generation $\gamma$-ray beam facility will enable new exclusive measurements of photodisintegration of few-nucleon systems with a precision that provides sensitivity to three-nucleon interactions.

- Nuclear astrophysics: new $\gamma$-ray beam capabilities will enable measurements that contribute broadly to open questions in nuclear astrophysics, questions such as big-bang nucleosynthesis, helium burning in massive stars, and synthesis of heavy nuclei. One of the most important reactions in stellar modeling is ${ }^{12} \mathrm{C}(\alpha, \gamma){ }^{16} \mathrm{O}$. The rate of this reaction relative to the carbon forming reaction $3 \alpha \rightarrow{ }^{12} \mathrm{C}$ determines the fate of massive stars. 
The measurement of the rate of the ${ }^{16} \mathrm{O}(\gamma, \alpha){ }^{12} \mathrm{C}$ reaction at energies approaching the temperatures at the core of stars is a grand challenge in nuclear astrophysics.

- Fundamental symmetries: the beams at an advanced low-energy $\gamma$-ray beam facility will enable measurements of parity violating photodisintegration of few-nucleon systems. In particular, a measurement of parity violation (PV) in deuteron photodisintegration near threshold is sensitive to a nucleon-nucleon (NN) PV amplitude that is not accessible using other systems. Such measurements sample the short-range part of the NN interaction, providing unique quantities for comparison with calculations using lattice gauge theory.

- Applications: the intense mono-energetic $\gamma$-ray beams at low-energy NGLCGS facilities will enable photonuclear reaction measurements important for technologies and techniques used in homeland security and nuclear safeguards. Programs to develop fielddeployable system will benefit by having a target areas equipped for evaluating concepts for $\gamma$-ray beam interrogation of cargo, nuclear fuel, and special assemblies at these facilities. The new beam capabilities at advanced $\gamma$-ray sources will also create opportunities for applications in medicine.

\subsection{Findings of advanced accelerator and light-source technologies working group}

Details of the various technology options considered are presented in the chapter on accelerator concepts for NGLCGS facilities. A summary of the findings of the working group is below.

It is unlikely that a single $\gamma$-ray beam source can meet the requirements of both the lowenergy $\left(E_{\gamma}<20 \mathrm{MeV}\right)$ and medium-energy $\left(E_{\gamma}>60 \mathrm{MeV}\right)$ parts of the field as described in the working group summaries above and in the science sections of this document. In the options discussed, the $\gamma$ rays are produced by Compton scattering of electrons from photons in an optical cavity that is pumped with an external laser. Two options for the electron beam accelerators for the low-energy $\gamma$-ray source were considered: a storage ring and an energyrecovery linac with superconducting RF (SRF) cavities. For the medium-energy $\gamma$-ray source, a storage ring was the primary option. There is confidence that a high-quality electron beam with low emittance and low energy spread can be maintained in modern storage-ring lattices, thereby enabling production of $\gamma$-ray beams with low energy spread. The new facility construction cost of the storage-ring option for either a low-energy or medium-energy next-generation Compton $\gamma$-ray source will be about $\$ 150 \mathrm{M}$. The working group cautions that this estimate is extremely uncertain; it is intended only to set the scale within about a factor of two. For the low-energy sources, less expensive options, such as upgrades to existing facilities, were also discussed.

\subsection{Recommendations}

The working groups through consensus make the following recommendations. The order of this listing is not prioritized.

- High intensity $\gamma$-ray beams with circular and linear polarizations will be produced at next-generation $\gamma$-ray sources by Compton scattering of photons from relativistic electrons inside a high finesse optical cavity. The optical cavity will be pumped by a laser system with high precision control of beam polarization. The electron beam accelerator will use proven technologies, either a storage ring or an energy-recovery linac. The main technological challenge is the production of reliable optical cavities with the technical specifications required for the next-generation $\gamma$-ray sources.

The highest priority $R \& D$ work for the next-generation $\gamma$-ray source should be the development of high finesse optical cavities and the associated laser and optical systems. 
It is important for this work to include testing and optimization of the cavity under $\gamma$-ray production conditions.

- For photopion production experiments, small angle electron scattering (virtual photon tagging) with intense electron beams was discussed as an alternative to measurements using tagged bremsstrahlung sources or $\gamma$-ray beams produced by Compton scattering. This alternative technique would be implemented with high-current electron beams, possibly in a storage ring, and with thin targets that allow detection of the low-energy charged particles produced in the reaction. This method promises to be much more effective than conventional photon tagging techniques.

R \& D should be supported to develop an alternative to Compton scattering for producing $\gamma$ rays with energies above the pion-production threshold. A system for virtual photon tagging in small-angle electron scattering is a promising candidate for such studies.

- Low-energy QCD phenomena will be explored at NGLCGS facilities with unprecedented precision at energies from below the pion-production threshold through the delta(1232) resonance region. The core experimental programs will involve Compton scattering to study the spin-dependent electromagnetic response of nucleons and near-threshold photopion production. Both programs require polarized beams and targets. The measurements enabled by NGLCGS facilities, together with advances in calculations using LQCD and QCD-based effective field theories, will explore the QCD origin of nucleon structure and charge-symmetry breaking.

Investments in polarized targets are needed to prepare for experiments at the NGLCGS facilities.

Investments in nuclear theory are needed to support the planning and analysis of lowenergy QCD experiments at the NGLCGS facilities.

- To ensure full realization of the scientific potential of an NGLCGS it is crucial that a strong theory effort in this area be maintained as the machine concept is developed and implemented. This will facilitate planning for experiments that optimally realize the scientific goals articulated in this document and continue the strong tradition of synergy between theory and experiment in low- and medium-energy photonuclear physics. Mechanisms that will ensure there is a strong international theory community working on this physics that is fully engaged include workshops with small lead times and durations of up to a month and partial support for postdoctoral researchers and/or graduate students working on theory projects related to the NGLCGS. In addition, computing resources can help address the challenge of solving QCD in this regime and contribute to the 2015 long-range plan's recommendation of 'new investments in computational nuclear theory that exploit US leadership in high-performance computing'.

- The ${ }^{12} \mathrm{C}(\alpha, \gamma){ }^{16} \mathrm{O}$ reaction helps regulate the efficiency of helium burning in massive stars and ultimately determines the mass of the iron core in the incipient supernova. The uncertainty in the measured cross section for this reaction substantially limits our understanding of the late stages of the life of massive stars and the details of the nucleosynthesis under the explosive conditions of supernovae. The beams at NGLCGS facilities will enable measurements of the ${ }^{16} \mathrm{O}(\gamma, \alpha){ }^{12} \mathrm{C}$ reaction that determine the reaction rate of $\alpha$-particle capture on ${ }^{12} \mathrm{C}$ at center-of-mass energies lower than have been achieved with other techniques. Measurements of angular distributions require thin targets to allow detection of the $\alpha$ particles along with charged-particle detectors with wide angle coverage. Options include 


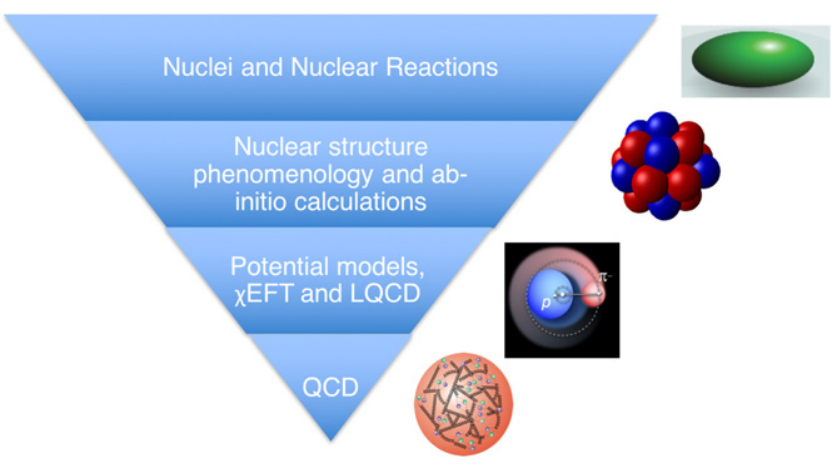

Figure 1. Schematic diagram for a coherent theoretical treatment of nuclear systems starting from high energies (at the bottom), where perturbative QCD can be applied, and ending with low-energy nuclear phenomena, where mean-field potential models are most efficient. Adapted from [18]. CC BY 4.0.

time projection chambers with optical and charge readout and silicon strip detectors with thin solid or gas targets.

Investments in active targets, such as time projection chambers, are needed to carry out the highest impact nuclear astrophysics measurements at NGLCGS facilities.

\section{Introduction}

Over the last decade, substantial progress has been made in developing formalisms and computational methods that contribute to theoretically coherent descriptions of nuclear phenomena with origins in QCD. An ultimate goal would be to describe nuclear matter, over wide distance and energy scales, with a QCD Lagrangian. Achieving this aim will likely require the effort of generations of scientists, as is often the case for grand challenges in science and technology. Figure 1 shows a schematic diagram of a plausible hierarchy for organizing the theoretical treatments of nuclear systems spanning a variety of phenomena. The scheme starts at high energies, where the most fundamental degrees of freedom are quarks and gluons, and progressively evolves in complexity. This diagram is intended only to represent gross features that should be included in a coherent picture of strongly interacting matter. At the top of the diagram, meanfield potentials that describe nuclear structure properties, the collective motion of nuclei, and nuclear reactions should be derived from residual strong interactions between nucleons. $A b$ initio calculations of the structure of light nuclei [1-4] and few-nucleon reaction dynamics [5] enable refinement of two-nucleon and multi-nucleon interactions using effective degrees of freedom. Current theoretical tools for describing the strong nuclear force (two- and threenucleon interactions) include semi-empirical potential models (see, e.g., references [6-10]), effective field theory (EFT) formulations of two-nucleon $(2 \mathrm{~N})$ and three-nucleon $(3 \mathrm{~N})$ interactions [11-14], and LQCD calculations of few-nucleon systems (see, e.g., references [15, 176]). Descriptions of the collective properties of nucleons in terms of effective field theories (see, e.g., references $[11,12])$ and LQCD (see, e.g., references $[17,176]$ ) are steps toward bridging gaps between QCD and theoretical treatments of few-nucleon systems. The remainder of this chapter introduces photon beams and their applications in nuclear science. References are provided in the later chapters, where these subjects are developed in detail. 
Photon beams are a highly-selective probe of the electric-charge and magnetism distributions of nuclei and nucleons. Measurements of photon scattering and photon-induced reactions provide information on the collective response of the internal degrees of freedom of composite nuclear objects, as depicted in figure 1. For almost a century, bremsstrahlung $\gamma$-ray beams have been the workhorse for investigating nuclear and nucleon structure and for measuring photon-induced nuclear reactions. These beams have the advantage of allowing measurements to be performed over a broad energy range in a single experiment, thereby providing information about the energy dependence of phenomena. However, the continuous energy nature of bremsstrahlung $\gamma$-ray beams has the disadvantage of limiting measurement sensitivity due to backgrounds created by the photons with energies outside the region of interest. A complementary tool is monoenergetic $\gamma$-ray beams, such as those created by laser Compton photon sources. The narrow energy bandwidth of laser Compton $\gamma$-ray beams enhances the signal-tobackground ratio in comparison to what is achievable using bremsstrahlung beams. In addition, the high beam polarization available for both linear and circular polarization in the $\gamma$-ray beams produced by laser Compton photon sources enables unambiguous determination of the spin and parity of excited nuclear states and facilitates studies of the spin structure of nucleons. Over the last several decades, laser Compton $\gamma$-ray beam facilities have provided intense polarized and nearly mono-energetic $\gamma$-ray beams for research programs in basic and applied nuclear physics. The facilities include GRAAL at the European Synchrotron Radiation Facility in Grenoble, LEGS at Brookhaven National Laboratory, LEPS at the SPring-8 facility, NewSUBARU at the University of Hyogo, and $\mathrm{HI} \gamma \mathrm{S}$ at the Triangle Universities Nuclear Laboratory. New laser Compton $\gamma$-ray beam facilities under construction include ELI-NP in Romania and the $\gamma$-ray beam line at the SSRF in China. The current generation of laser Compton $\gamma$-ray beam sources are based mostly on either a single-pass light pulse from an external laser scattered off a single-pass electron beam bunch or a single-pass laser pulse scattered off electron beam bunches circulating in a storage ring. The technique of intracavity Compton scattering of electrons circulating in a storage ring that is employed at $\mathrm{HI} \gamma \mathrm{S}$ is indicative of the technologies to be used in next-generation laser Compton $\gamma$-ray sources. Combining the high beam current of storage rings with the high photon density inside optical cavities can potentially produce monoenergetic $\gamma$-ray beams with intensities more than three orders of magnitude higher than $\mathrm{HI} \gamma \mathrm{S}$, which is currently the most intense laser Compton source in the world.

This whitepaper describes research opportunities that would be opened up by the $\gamma$-ray beam capabilities at next-generation laser Compton $\gamma$-ray beam facilities. Its content is based mainly on discussions and findings of The International Workshop on the Next Generation Laser Compton Gamma-Ray Beam Facility that was held November 17-19, 2016 in Bethesda, MD. The workshop brought together both researchers from the international low-energy and medium-energy nuclear physics communities, along with accelerator physicists and experts in optics. The accelerator and optics experts anchored this exercise into the constraints of beam performance parameters achievable with technologies that can be implemented in the coming decade. For both practical reasons and scientific considerations, the upper energy reach of the $\gamma$-ray source was limited to $500 \mathrm{MeV}$. Within these boundaries, the broad areas of nuclear physics considered correspond to the upper three panels of figure 1. It is probably fair to state that most participants began the workshop with the belief that one laser Compton source would be capable of serving both low-energy experiments, with $\gamma$-ray beam energies $\left(E_{\gamma}\right)$ below about $20 \mathrm{MeV}$, and medium-energy experiments, with $E_{\gamma}>60 \mathrm{MeV}$. The questions addressed in lowenergy and medium-energy $\gamma$-ray experiments have considerable intellectual overlap and the intense, monoenergetic $\gamma$-ray beams of an NGLCGS would facilitate progress in both regimes. However, the $\gamma$-ray source considerations mean that research at low and medium energies likely must be carried out at two different facilities. For this reason, the discussion of the research 


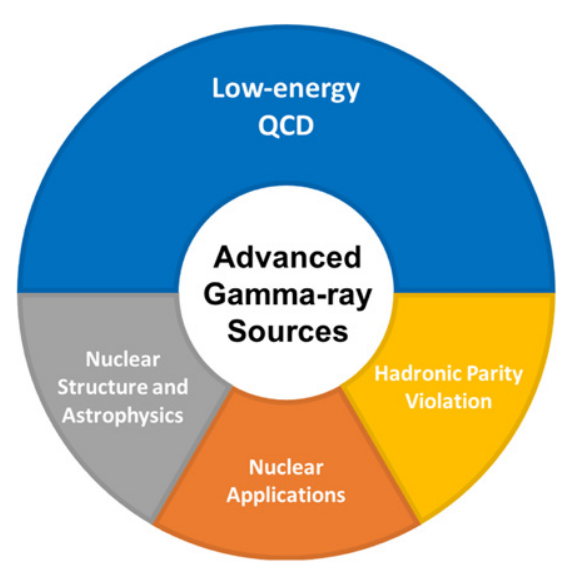

Figure 2. Illustration of the research areas impacted by advanced $\gamma$-ray source technologies. The upper half of the graphics represent the main research at $\gamma$-ray beam energies from about $60 \mathrm{MeV}$ to $350 \mathrm{MeV}$. The lower half represents the research areas covered at energies below $20 \mathrm{MeV}$.

opportunities created by laser Compton $\gamma$-ray sources is organized by the two energy ranges of the facilities presented at the workshop, $E_{\gamma} \leqslant 20 \mathrm{MeV}$ and $60 \mathrm{MeV} \leqslant E_{\gamma} \leqslant 350 \mathrm{MeV}$. The areas explored in each energy region are illustrated in figure 2. While low-energy QCD is mentioned explicitly only in the upper half of the circle it is also strongly connected to the proposed research on PV in hadronic systems and the studies of few-nucleon systems and light nuclei that compare $a b$ initio calculations to data. Indeed, since all nuclear dynamics is underpinned by QCD low-energy QCD in fact pervades all the opportunities that would be created by an NGLCGS. These include PV in hadronic systems and studies of few-nucleon systems and light nuclei using ab initio calculations. Details of the opportunities are presented in the topical chapters that follow. A synopsis of each topical discussion is given below.

The main opportunities at energies below $20 \mathrm{MeV}$ are presented in section 3 and are in the areas of nuclear structure (including photon-induced fission), nuclear astrophysics, and PV in few-nucleon systems and light nuclei. Studies of collective modes of excitation can provide information about short-range correlations between nucleons in nuclei and can reveal features of the nuclear EOS. The giant dipole resonance (GDR) dominates the nuclear collective response and is well understood. The rotational (rigid rotor) and vibrational (scissor) modes at energies below about $4 \mathrm{MeV}$ have been extensively studied and are described by well-established models. The nuclear excitations on the low-energy tail of the GDR, above about $5 \mathrm{MeV}$, and below the particle separation energy are not well characterized. Much of the difficulty in understanding the nature of the excitations in this energy region is associated with distinguishing between effects due to the GDR and those due to other mechanisms. The dipole excitation strength in excess of the GDR tail is referred to as the pygmy dipole resonance (PDR). The generally accepted mechanism for the PDR is the vibration of a neutron skin off an isoscalar core. If this picture is correct, a study of its strength as a function of isospin and $A$ should provide information about the density dependence of the symmetry energy term in the nuclear EOS. The beam intensities available at the next-generation laser Compton $\gamma$-ray sources will enable nuclear resonance florescence (NRF) measurements on nuclei at extremes of isospin where the natural abundance is low and consequently target material is 
sparse. In addition, the beam at next-generation laser Compton $\gamma$-ray sources will enable thorough measurements of the $M 1 \gamma$-ray strength in nuclei. Special attention will be given to nuclei in the mass vicinity of isotopes used in double-beta decay experiments. The beams at the nextgeneration laser Compton $\gamma$-ray source will also enable photon-induced fission measurements at energies near threshold, where the cross section is low, and the study of low-yield fragments. Such experiments will provide new information about the potential energy surface that governs the evolution of the fission process. Examples of the initial nuclear structure research made possible by the NGLCGS facilities are described in section 3.1.

The beams at the next-generation laser Compton $\gamma$-ray sources will enable strategic measurements of a variety of NRF and photon-induced nuclear reactions that contribute to modeling p-process, s-process and r-process nucleosynthesis. However, the highest impact contribution to nuclear astrophysics will be in determining the reaction rate for alpha-particle capture by carbon via the ${ }^{12} \mathrm{C}(\alpha, \gamma){ }^{16} \mathrm{O}$ reaction in massive stars during the alpha-burning stage. The goal for all measurements of this reaction is to determine the cross section at a center-of-mass energy of $300 \mathrm{keV}$. In $\gamma$-ray beam facilities, the time reversed reaction ${ }^{16} \mathrm{O}(\gamma, \alpha){ }^{12} \mathrm{C}$ will be measured, and detailed balance will be applied. This measurement will be the flagship nuclear astrophysics experiment at advanced $\gamma$-ray sources, and, as such, should be measured using a variety of techniques. Nuclear reaction rate measurements enabled by the NGLCGS facilities that are at key points in stellar nucleosynthesis networks are discussed in section 3.2.

In the area of fundamental symmetries, the primary focus will be on studying PV in hadronic systems. Such PV is a measure of the weak interaction inside systems of strongly interacting particles. The ultimate goal is to measure PV in photodisintegration reactions of few-nucleon systems, such as ${ }^{2} \mathrm{H}$ and ${ }^{3} \mathrm{He}$. Because these measurements must be performed near the reaction threshold energy, and because the parity violating asymmetry is extremely small $\left(\sim 10^{-7}\right)$, these experiments make the most stringent demands on the source-performance parameters. That is, a $\gamma$-ray source that delivers beam intensities an order of magnitude below what is required for these experiments will enable all the nuclear structure and nuclear astrophysics discussed in this whitepaper. Measurements of parity violating photoabsorption asymmetries on parity doublets in light nuclei will be used to assess beam and instrumentation asymmetries in the early stage of developing the capabilities for performing $10^{-7}$ asymmetry measurements. For this purpose, nuclei with photoabsorption asymmetries of $10^{-4}$ to $10^{-3}$ will be selected. Hadronic PV (HPV) studies using photon-induced reactions are described in section 3.3.

In addition to the basic science research enabled by the NGLCGS facilities, these $\gamma$-ray sources will provide capabilities that support technology and techniques development work for nuclear security. How such facilities might be used in efforts to advance $\gamma$-ray beam interrogation systems is outlined in section 3.4.

QCD is the fundamental theory of the strong nuclear force. When written in terms of quark and gluon degrees of freedom, it is deceptively simple. Indeed, QCD's asymptotic freedom guarantees that this form of the theory describes strong interactions at sufficiently high energy. However, in that regime, QCD does not bind quarks and gluons into neutrons and protons. The task of fully and rigorously deriving the presence and properties of neutrons, protons, and nuclei from the Standard Model (SM) remains a grand challenge for physics, requiring us to understand the emergence of new degrees of freedom as the theory becomes strongly coupled. Without such an understanding, treatments of the nuclear force based on the fundamental theory will remain elusive, and so too will the ability to model nuclei in a reliable manner that is grounded in QCD.

High-intensity beams of polarized $\gamma$ rays in the energy range of $60-300 \mathrm{MeV}$ provide a unique opportunity to test our understanding of the emergence of neutron and proton structure from the SM. In this regime, a description of experimentally observed phenomena in terms of 
neutrons and protons and their low-lying excitations is efficient, thanks to the chiral symmetry of QCD.

The initial research opportunities in nucleon structure and low-energy QCD at NGLCGS facilities are described in section 4 . One of the main goals at these energies will be to map out the spin-independent and spin-dependent polarizabilities starting at energies below the pion production threshold, extending over the pion production threshold energy, and continuing through the delta(1232) region. These measurements will be carried out using Compton scattering from unpolarized and polarized targets. The other major aim in the medium-energy domain is precision near-threshold pion photoproduction data. Such data would provide the opportunity to extract the s-wave $\pi^{0} \mathrm{p}$ scattering length and so gain a new window on the QCD origin of charge-symmetry breaking.

In the limit of vanishing up-, down-, and strange-quark masses, the QCD Lagrangian admits a global chiral symmetry: $\mathrm{SU}(3)_{\mathrm{L}} \times \mathrm{SU}(3)_{\mathrm{R}}$. This symmetry is broken spontaneously, which implies the existence of eight pseudoscalar massless Goldstone bosons. Furthermore, since the quark masses are finite (but small), these Goldstone bosons acquire a small mass and are identified with the pions, kaons, and etas. The interaction of these Goldstone bosons with themselves or with matter fields such as nucleons is weak. This allows for a systematic low-energy expansion in terms of small momenta and quark masses: chiral perturbation theory. Chiral perturbation theory $(\chi \mathrm{PT})$ describes meson-meson interactions in this energy domain with high precision. It also describes the interactions of mesons with nucleons at very low energies. Incorporating the $\Delta(1232)$ as an explicit degree of freedom in the theory yields a chiral EFT $(\chi \mathrm{EFT})$ that describes processes at energies below the chiral symmetry breaking scale $(\Lambda \approx 1 \mathrm{GeV})$ via an expansion in a small, dimensionless parameter. This allows reliable quantification of residual theory uncertainties. Thus $\chi$ EFT exploits the chiral symmetry of QCD in order to rigorously connect QCD with the phenomenology of nuclear and particle physics. Because $\chi \mathrm{EFT}$ encodes the consequences of the SM for low-energy processes involving photons, pions, and nucleons, it can be used to test whether observed strong-interaction phenomena are consistent with the SM. In particular, $\chi$ EFT allows us to elucidate the experimental consequences of the pattern of chiral-symmetry breaking in QCD.

Accelerator concepts for the NGLCGS are described in section 5. Two options for the accelerator configuration were discussed for energies below $20 \mathrm{MeV}$ : an energy-recovery linac with SRF cavities or a storage ring. Only the storage-ring option was presented for energies above $60 \mathrm{MeV}$. In all source configurations, the Compton scattering occurs inside an optical cavity that is pumped by an external laser. The electron source, accelerator structures, and storagering lattices that meet the required technical specifications for source performance are robust and can be implemented to order. The situation is not as well established for the optical cavity. R \& D is needed to develop an optical cavity design that can meet the power, stability, and reliability requirements of the next-generation laser Compton $\gamma$-ray source.

\section{Nuclear physics research and applications with gamma-ray beams below $20 \mathrm{MeV}$}

\subsection{Nuclear structure}

The landscape for the response of nuclei to electromagnetic radiation as a function of excitation energy is shown schematically in figure 3. The electric GDR is the dominant collective response of nuclei. This mode can be modeled as the oscillation of the proton matter distribution against the neutron distribution. In the classical interpretation, the width of the cross-section enhancement associated with the resonance provides information about the damping forces, i.e., the 


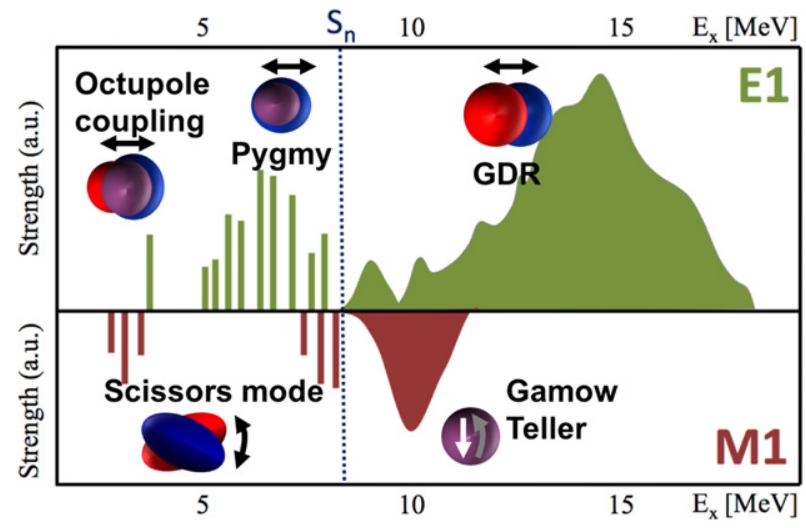

Figure 3. Diagram of the landscape of the response of nuclei to photon absorption.

viscosity of the nuclear matter. The nature of the nuclear response at excitation energies below the GDR around the particle in thresholds is still rather unclear and is the topic of considerable theoretical and experimental work [19]. The excitation in this region has been observed to be largely electric dipole $(E 1)$. This additional structure below the GDR has been shown to be a common feature in the $E 1$ response of atomic nuclei, and is generally referred to as the PDR. The $E 1$ response offers the possibility of studying the EOS of neutron matter [20-25]. Also the $\gamma$-ray absorption cross section in the PDR energy region influences nuclear reaction rates in stars [26-29]. In deformed nuclei, the collective nuclear dipole response at excitation energies below the PDR region is mainly due to magnetic dipole (M1) transitions, which can be described as a scissor motion. The $\left(\gamma, \gamma^{\prime}\right)$ reaction, which is referred to as nuclear resonance fluorescence (NRF), is the most effective approach for probing these features of nuclear structure.

Nuclear fission is a complex process in which the collective motion of nucleons results in a strong change in the shape of the nucleus, ultimately leading to a breakup into fragments. Photon-induced fission can provide unique insight into the evolution of the potential energy surface during the fission process as it progresses from photon absorption through to the scission point. Measurements of fission product yields of isotopes with different half lives provide information on the evolution of the nuclear deformation and breakup of the nucleus during the fission process.

The high intensity, nearly monoenergetic, and linearly polarized photon beams at a nextgeneration laser Compton $\gamma$-ray beam facility will enable high-precision photonuclear reaction measurements. Research opportunities for NRF and photofission measurements are described in this section.

3.1.1. Nuclear resonance fluorescence. The nuclear resonance fluorescence (NRF) reaction is one of the work horses in the investigation of the dipole response of atomic nuclei. The method enables the extraction of intrinsic properties of excited states such as spin, parity and transition widths in a model independent way. Furthermore, due to the low momentum transfer of photons, primary dipole transitions are induced, i.e., in even-even nuclei states with $J=1^{ \pm}$ are populated. This makes NRF an excellent reaction to systematically study the phenomena in the dipole response of nuclei, such as the PDR and the scissors mode over a large range of nuclei. 


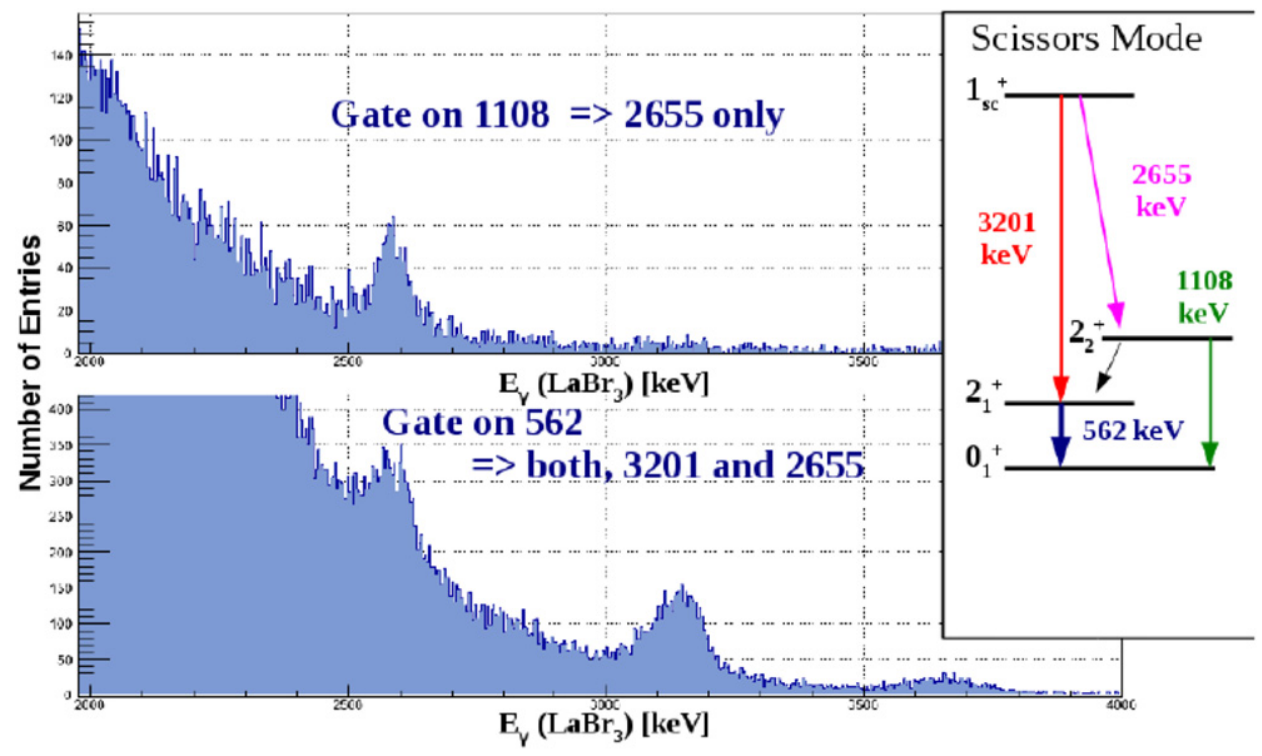

Figure 4. Example of $\gamma \gamma$ coincidence spectroscopy. The spectra show projections of $\mathrm{LaBr}_{3} \times \mathrm{LaBr}_{3}$ matrices after gating on transitions from the first and second $2^{+}$states in ${ }^{76} \mathrm{Ge}$. The corresponding level scheme is given on the right-hand side. The scheme is an example for any dipole excited state with arbitrary intermediate levels between the $J=1$ excited state and the ground state.

Thus far, mostly the decay branch back to the ground state as been investigated with the NRF reaction. However, the detailed decay pattern of excited states is often connected to important details in its nuclear structure. The decay widths to the individual lower-lying excited states or the ground state are directly linked to the corresponding transition matrix elements. Thus, the individual decay channels are sensitive to different components of the nuclear-state wave function. For example, in the case of transitions to lower-lying excited states, the de-excitation takes place via a different component in the wave function than the excitation from the ground state. Therefore, the observation of these transitions and the determination of the branching ratios reveal important experimental information that tests modern nuclear-structure models.

The method of $\gamma \gamma$ coincidences in the spectroscopy of the decay of excited states has been proven to be a powerful tool for determining even small branching ratios to excited low-lying states [30,31]. The principle is illustrated in the left part of figure 4. After excitation by photoabsorption, the high-lying state at excitation energy $E_{x}$ may de-excite either directly to the ground state with width $\Gamma_{0}$ or via an intermediate state with width $\Gamma_{\mathrm{i}}$. By detecting the two emitted $\gamma$ rays in coincidence, even small branching ratios $\Gamma_{\mathrm{i}} / \Gamma_{0}$ can be determined with good precision, since the background is strongly suppressed. This technique allows the branching of dipole-excited states to individual lower-lying levels to be determined, thus greatly aiding in building up complex level and decay schemes. An example is shown in figure 4 . The right-hand side of the figure provide, the level structure for a measurement on ${ }^{76} \mathrm{Ge}$, where the decay of the $1^{+}$scissors mode has been investigated [32]. The $1^{+}$state at $3.763 \mathrm{MeV}$ is populated by photo-excitation. Gating on the decays of the $2_{1}^{+}$and $2_{2}^{+}$states, as shown on the left side of the figure, allows the order of the $\gamma$-ray transitions in the decay to be determined. 
The combination of large-volume $\mathrm{LaBr}_{3}$ and high-resolution HPGe detectors opens the way for $\gamma \gamma$-coincidence measurements with sufficient statistical accuracy to track $\gamma$-ray decay cascades. High-resolution HPGe detectors allow for a separation of individual $\gamma$-ray transitions from dipole excited states in regions where the level density is not yet too high. Even in regions with a high level density, individual states can sometimes have substantial excitation strength and are, thus, separable from the otherwise rather continuous spectrum. This provides a way to normalize spectra to complementary data sets obtained using bremsstrahlung beams. Nevertheless, most dipole-excitation strength in regions of high level density is so strongly fragmented that individual states cannot be resolved. In this case, the large-volume $\mathrm{LaBr}_{3}$ detectors have a significant advantage over HPGe detectors because of their higher detection efficiency. This high efficiency allows the fragmented strength in the beam region to be separated from the background and enables integrated branching strengths to be determined. In deformed nuclei, where the first-excited-state energies are very low, decays to the ground state and the first excited state are close in energy, posing a challenge to the accelerator team to deliver beams with high intensity and low energy spread to the target. Deconvolution methods have recently been developed and applied to aid in extracting decays out of the detector response (figure 5), see e.g. [33, 34].

With the combination of HPGe and $\mathrm{LaBr}_{3}$ detectors, the high efficiency of the latter provides sufficient statistics to observe branching decays from higher-lying states, either in lowresolution $\mathrm{LaBr}_{3}$ or even in high-resolution HPGe spectroscopy, as demonstrated in [30, 31]. Especially at low energies, electric quadrupole excitation probabilities can be sufficiently large for observation, and can be separated from the dipole strength making use of angular distribution and correlation measurements using arrays of $\mathrm{HPGe}$ and $\mathrm{LaBr}_{3}$ detectors. In certain cases even the mixing ratio of $M 1 / E 2$ transitions can be measured. This recently facilitated a first measurement of the isovector quadrupole strengths in ${ }^{156} \mathrm{Gd}$ [35], for example.

The capabilities of the next-generation laser Compton $\gamma$-ray source combined with modern $\gamma$-ray detectors will enable measurements that address a broad range of physics through photoexcitation studies. The topics include multi-phonon structures such as quadrupole-octupole coupled states and the search for rotational isovector scissors excitations; the investigation of the PDR along with its structural evolution and fingerprints in going from spherical to deformed regions; and detailed spectroscopic studies of the $M 1$ spin-flip resonance. Therefore, the physics topics to be addressed embrace shape coexistence, octupole correlations, isovector excitations, neutron skins, and photon strength functions (PSFs), often with strong relevance for the fields of nuclear astrophysics and weak-interaction physics such as neutrinoless double-beta decay and neutrino scattering.

3.1.1. Shape coexistence and link with physics with radioactive beams In the context of nuclear structure studies, a direct link between research carried out with $\gamma$-ray beams and with radioactive beams should be briefly discussed. Shell structure is a cornerstone in the description of atomic nuclei as many-body quantum systems. The first data on neutron-rich nuclei far from stability have provided evidence that shell structure evolves with neutron excess. For example, some well-known magic numbers disappear while others appear. Progress in the description of these exotic nuclei is due in no small part to the development of new experimental techniques that have gone hand in hand with the introduction of new theoretical concepts. It has been shown that effective single-particle energies are significantly modified in neutron-rich systems through the action of the monopole component of the proton-neutron tensor force [36]. Furthermore, multi-particle multi-hole excitations trigger shell evolution as a function of spin and excitation energy [37]. In this picture, the occupation of specific deformation-driving orbitals leads to changes in nuclear shapes and to the possibility of shape coexistence. The 


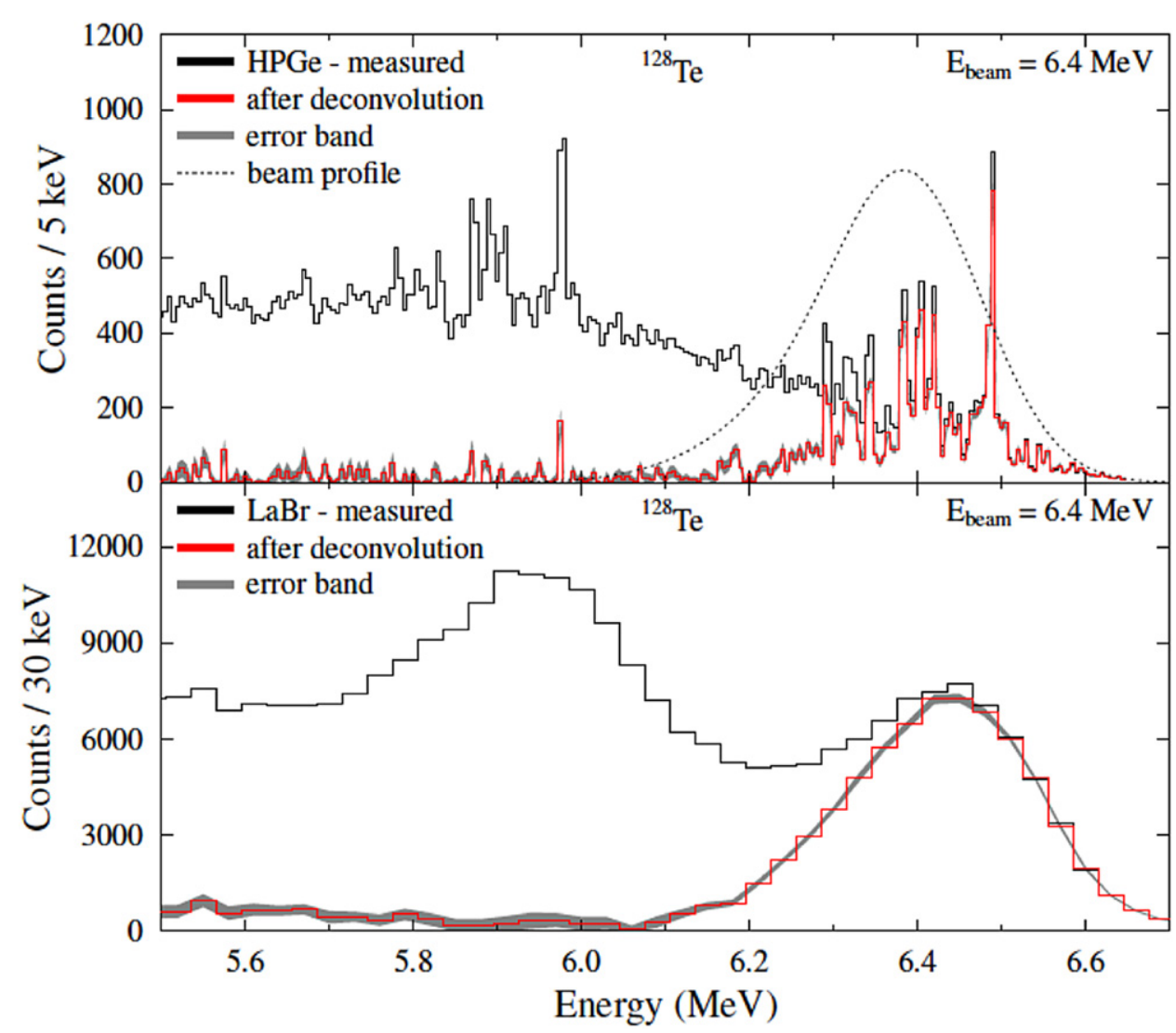

Figure 5. HPGe (top) and $\mathrm{LaBr}_{3}$ (bottom) spectra from singles $\gamma$-ray spectroscopy of ${ }^{128} \mathrm{Te}$ at a beam energy of $6.4 \mathrm{MeV}$. The black histograms show the actual spectra, and the red ones are the result of the deconvolution, which includes correcting for the detector response. The beam profile is indicated by a dashed curve in the upper panel. Reproduced with permission from [33].

region between $Z=20, N=20{ }^{40} \mathrm{Ca}$ and $Z=28, N=50{ }^{78} \mathrm{Ni}$ provides a good illustration of the synergy between experiments at the national user facilities and measurements with $\gamma$ ray beams. For example, triple shape coexistence has recently been discovered in neutron-rich ${ }^{66,68,70} \mathrm{Ni}[38]$. These $0^{+}$states are the result of multi-particle multi-hole excitations. Some of these levels, in particular those associated with prolate deformation, are understood as proton excitations that are predicted to be present in the stable ${ }^{60,62,64} \mathrm{Ni}$ as well [38], and the evolution of these states' excitation energy with $\mathrm{N}$ is a matter of much theoretical debate. The issue can be addressed with intense $\gamma$-ray beams: in a first phase, all the low-spin excitations can be mapped out. Following this discovery phase, information on the wave function of the states will be obtained from state lifetime measurements, and from the determination of decay branching ratios. Furthermore, excitations built on the excited $0^{+}$levels will be delineated and their properties characterized. Other regions of the nuclear chart lend themselves to similar studies. Specifically, shape changes and shape coexistence phenomena, driven by multi-particle multihole excitations are predicted to occur in the $\mathrm{Sr}-\mathrm{Mo}-\mathrm{Zr}$ region as well as in the vicinity of doubly-magic ${ }^{208} \mathrm{~Pb}$. 


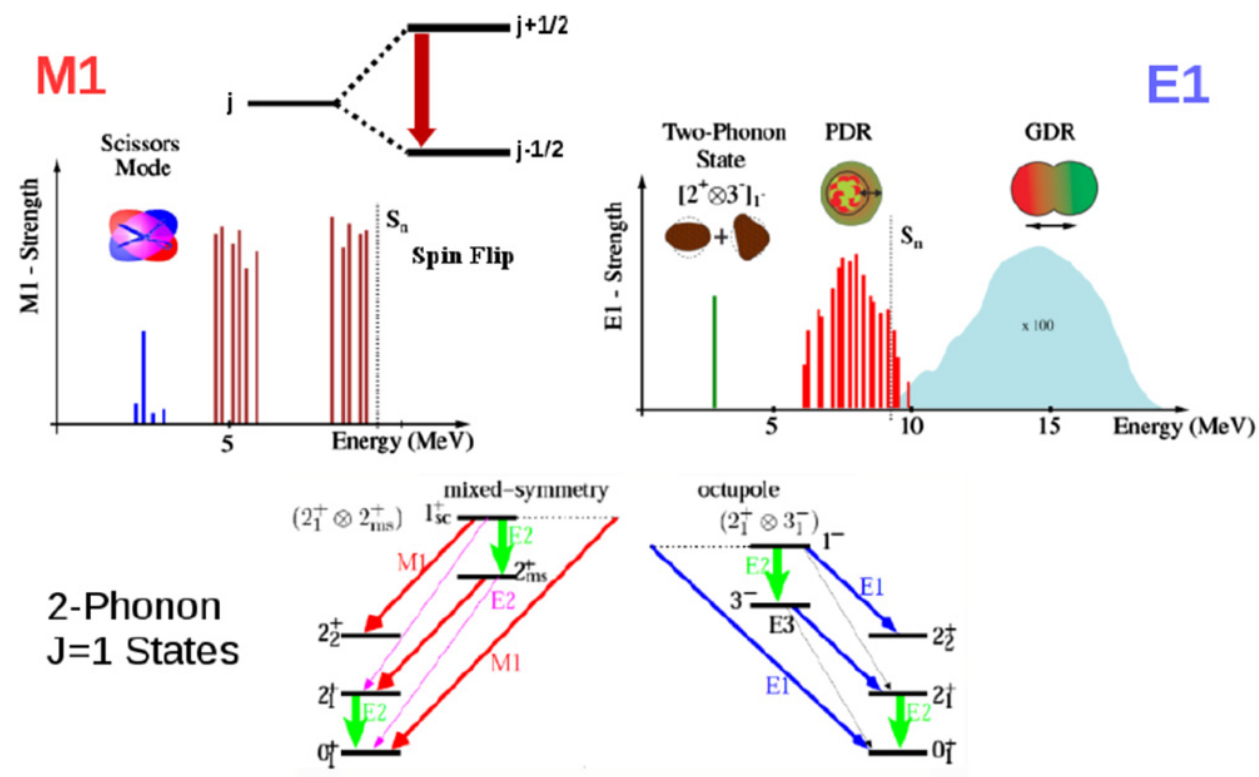

Figure 6. Schematic diagram of nuclear excitations involving multiple phonons and illustrating mixed symmetry and octupole excited states.

3.1.1.2. Multi-phonon and rotational low-lying states At low energies, in spherical or nearspherical nuclei, excited states are often formed by phonon excitations. For example, the first $2^{+}$state is identified with a quadrupole phonon, and the first $3^{-}$state with an octupole phonon. Such phonons can be coupled, leading to multiplets of multi-phonon states built from like phonons, or from mixtures of, e.g., quadrupole and octupole phonons. As such, the quadrupole-octupole coupled quintuplet of states, $1^{-} \ldots 5^{-}$contains an $E 1$-excited $1^{-}$state which typically is the first excited $1^{-}$state, located at roughly the sum energy of its constituents and carrying most of the $E 1$ strength at low energies. (See the schematic diagram of $\gamma$-ray transitions associated with multiple-phonon de-excitation is given in figure 6.) In addition to excitations where protons and neutrons move in phase, there are so-called mixed-symmetry states where at least one pair of protons/neutrons has a different phase [39]. This leads to additional states, such as a quadrupole-excited one-phonon mixed-symmetric $2_{\mathrm{ms}}^{+}$state. Again, the phonon coupling between the latter and the symmetric $2_{1}^{+}$phonon state leads to a multiplet of mixed-symmetry states, one of which is an $M 1$-excited $1^{+}$state, which is typically the strongest $M 1$ excitation at low energies of roughly 3 to $4 \mathrm{MeV}$. In rotational nuclei, this state evolves into the well-known scissors mode, where valence protons and neutrons are counter-oscillating in a scissors-like fashion.

Different mechanisms forming $E 1$ and $M 1$ excited states at such low energies can, however, compete with one another. For example, spin-flip excitations, where a nucleon is moved from an $\ell \pm 1 / 2$ orbital to its $\ell \mp 1 / 2$ partner, can generate $M 1$ excitation strengths similar to those of the scissors mode. In the $E 1$ sector, the generation of $E 1$ strength in valence spaces containing either no or a very limited number of opposite-parity orbitals is under much discussion, and there are other possibilities, such as the formation of alpha clusters (see below). Therefore, it is not only important to locate those dipole excited states and to determine their parity and decay strengths, it is also crucial to measure their decay pattern, which contains important information 
on their underlying structure. These tasks are uniquely facilitated by the capabilities at a nextgeneration laser Compton $\gamma$-ray source for measuring decay branches, parities, and relative excitation strengths. Furthermore, in rotational nuclei, band structures are expected on $J=1$ band-heads such as the scissors-mode state. Indeed, in recent work [35], a first candidate for the long-sought rotational $2_{\mathrm{sc}}^{+}$member of the scissors-mode band has been discovered. In addition, measurements of angular correlations made possible by arrays of $\gamma$-ray detectors with large angle coverage, high detection efficiency, and high energy resolution used in combination with linearly polarized monoenergetic $\gamma$-ray beams will enable measurements of multipole-mixing ratios. These, in turn, can reveal absolute isovector $E 2$ strengths. High quality data of this type are crucial in constraining effective charges in nuclear dynamics calculations.

Another aspect of dipole excited states has recently been found and is connected to weakinteraction physics. While the strong interaction dominates in nuclei, nuclei are also laboratories for studying the weak interaction through the possibility of $\beta$ or $\beta \beta$ decay. Of special interest for many experiments, and for the characterization of neutrinos in general, is the search for neutrinoless double-beta $(0 \nu \beta \beta)$ decay. The existence of this decay mode would identify the neutrino as a Majorana particle, one which is its own anti-particle. It would provide information about the absolute mass of the neutrino involved in the decay through the ratio of the measured decay rate and the calculated nuclear matrix element for the decay. Since many candidate isotopes (mother and/or daughter) for $0 \nu \beta \beta$ decay occur in mass regions near transitions from spherical to deformed shapes, their model description is challenging. Furthermore, isovector parameters are seldom known. NRF measurements can be used to extract information on phenomena such as shape coexistence and mixed-symmetry states from the observation of the scissors mode and its decays, especially its decays to the potentially mixed-configuration $0_{1,2}^{+}$ states (see reference [40]).

3.1.1.3. The E1 pygmy dipole resonance. The strongest and best-known $E 1$ mode in atomic nuclei is the GDR. It is a broad resonance structure peaking well above the particle-separation threshold energy. Therefore, in NRF experiments, only the low-energy tail of the GDR below the particle threshold energy is directly observable. Often, this tail is parameterized by a Lorentzian, eventually with modifications such as temperature dependence, e.g., parameterizations that directly use the so-called PSFs. PSFs have a direct relation to nuclear astrophysics, since they give a handle on the balance between particle-capture and photodisintegration processes in stellar nuclear synthesis. Over the last decade, observations have given rise to additional strength, on top of the low-energy tail of the GDR. This added strength is the so-called PDR [19]. This strength, typically a few percent of the GDR strength, appears around the neutron-separation threshold. It would directly influence PSFs and would, therefore, impact nucleosynthesis, which ultimately shapes the abundance patterns of elements [41].

Various studies of the PDR have involved magic or near-magic spherical nuclei. Usually, the origin of the PDR is thought to be an oscillation of a neutron skin against the proton-neutron saturated core. This picture is supported by several microscopic calculations, showing an excess of neutron transition densities near the nuclear surface. However, other possibilities exist. For example, the occurrence of alpha clusters on the surface, which would yield enhanced E1 strength, has been suggested [42]. Additional problems lie in the differentiation of PDR strength from the low-energy tail of the GDR. Alpha scattering seems to be a possible method for accessing this problem [43-45], because, compared to NRF data, low-energy E1 strength appears to split into isoscalar and isovector parts.

Other challenges lie in the determination of the total strength. For example, protonscattering data partly point to significantly more observed $E 1$ strength than photon-scattering data [46]. This may, at least in part, be due to branching transitions from dipole-excited states 
and many weak unresolved transitions, which would have been missed in earlier NRF experiments using bremsstrahlung beams. At the next-generation $\gamma$-ray source, the focus will be on identifying the strength distribution (state-by-state or average), measuring the parities as well as branching behavior of the excited states. From these data, more stringent conclusions can be drawn on the nature of the PDR, its overall strength, and therefore also on the determination of PSFs. In addition, the Brink hypothesis (the excitations that are built on the ground state should also be built on any excited state), which is part of the basis of the statistical model, can be tested in a model independent way using $\gamma-\gamma$ decay spectroscopy, as has been recently demonstrated [47].

A further challenge lies in the deformation degree of freedom. In the end, most nuclei on the chart of the nuclides are deformed, but our information on the evolution of the PDR toward deformed nuclei is sparse at best. Therefore, the experimental program should extend to address this problem. Indications of a potential splitting of the PDR into two parts with different $K$ quantum number have recently been observed in proton-scattering on ${ }^{154} \mathrm{Sm}$ [48]. This would be similar to the splitting of the GDR with respect to the two (or even three) axes of the nuclear body. However, the high degree of fragmentation in deformed nuclei renders a state-by-state analysis meaningless. Therefore, measurements that track the cascade of $\gamma$-ray decays are most useful. In addition, the very low energy of the first excited state in deformed nuclei requires a reduced energy spread of the photon beam compared to existing facilities in order to enable a clean separation of the decay intensity to the first excited state and the ground state. Such measurements over sizable isotope chains will be made possible by the beam capabilities at the next-generation laser Compton $\gamma$-ray beam facilities.

3.1.1.4. Alpha-cluster excitations. Below the particle-separation threshold, two underlying structures of $E 1$ excitations have been intensively studied in the last decade, the octupole modes $[49,50]$ and the PDR [19]. For both modes, a non-uniform distribution of protons and neutron generates $E 1$ transitions at lower energies. As pointed out in earlier sections, these two modes could have substantial impact on nuclear structure models and nuclear synthesis calculations. Another mode giving rise to enhanced $E 1$ strength at lower energies is the oscillation of an alpha cluster relative to the remaining bulk nuclear matter [42]. For light nuclei, alpha clustering is well-established [51], and its implications for the $E 1$ strength have been discussed. Also, in heavy isotopes such as ${ }^{212} \mathrm{Po}$, strong indications of a ${ }^{208} \mathrm{~Pb}+\alpha$ system have been observed [52]. Recently, the existence of alpha clusters in heavy nuclei was supported by adding four-particle correlations to shell-model calculations [53]. Because the formation of alpha clusters provides interesting insights into the formation of bosonic clusters in strongly coupled fermionic systems, identifying new signatures of alpha clustering in heavier nuclei is of general scientific interest.

Several enhanced $E 1$ transitions were observed in the neodymium chain by $\left(\gamma, \gamma^{\prime}\right)$ experiments up to $4 \mathrm{MeV}$ [54-56]. To shed light on the origin of the low-lying $J=1^{-}$states, $s p d f$ interacting-boson-model (IBM) calculations have been performed [57]. It has been proposed that the $p$-boson is related to $\alpha$-cluster configurations. By identifying the basis states by means of their boson contents $\left|\left[n_{s}\right],\left[n_{p}\right],\left[n_{d}\right],\left[n_{f}\right]\right\rangle$, the ratio $n_{p} / n_{f}$ permits an assignment of quadrupole-octupole or $p$-boson character to be associated with the excited states. The results of these calculations suggest the presence of alpha-cluster modes occurring at the surface of nuclei with atomic masses just above magic numbers. For example, in IBM calculations for ${ }^{144} \mathrm{Nd}$, a remarkable increase of $p$-boson contributions has been observed for energies up to the neutron-separation threshold.

3.1.1.5. The M1 spin-flip resonance. Although, overall, much less $M 1$ strength is expected in the region where the PDR occurs, there should be significant strength due to the spin-flip 
Table 1. Suggested beam parameters for NRF experiments.

\begin{tabular}{lc}
\hline Parameter & Value \\
\hline Energy & $2-20 \mathrm{MeV}$ \\
Flux $(\gamma / \mathrm{s})$ & $10^{9}$ at $1 \% \mathrm{FWHM}$ \\
Polarization & Linear \\
Diameter & $10 \mathrm{~mm}$ on target \\
Beam repetition rate & Few MHz \\
Beam pulse width & $<1.0 \mathrm{~ns}$ \\
\hline
\end{tabular}

resonance. The exact energy of this resonance depends on the underlying shell structure, but typically it coincides with the onset of the major $E 1$ strength distribution. Knowledge about this $M 1$ resonance is sparse, and present parameterizations are based on little available data. The challenges at hand are the identification of $M 1$ strength within the sea of $E 1$ excited states. In cases of low fragmentation, this can be done with a state-by-state analysis of excited-state parities, but at high fragmentation, one has to choose an average approach. The best way to filter out $M 1$ strength in those energy regions is the use of photon-scattering with a linearly polarized beam, such as those available at laser Compton $\gamma$-ray sources.

The impact of such higher-lying $M 1$-excited states has different facets. On the one hand, they will provide constraints to microscopic calculations, since the occurrence of spin-flip transitions involving particles moving between $\ell \pm 1 / 2$ partner orbitals, usually across a major nuclear shell. In addition, states excited by a spin-flip transition are those which are populated through Gamov-Teller transitions in $\beta$ decay. On the other hand, such $1^{+}$states will directly influence PSFs in even-even nuclei, since decays between $1^{-}$(GDR/PDR) states and the spin-flip states are allowed, changing the overall shape of the PSFs. More reliable data on the $M 1$ response can, therefore, serve as a test of parity asymmetry. (Usually the same number of positive and negative parity states is assumed.)

Again, $M 1$ strength, this time at energies above the scissors mode, can prove important for neutrino physics. Specifically, there are detectors, such as the molybdenum-based MOON detector [58], which are in the planning and construction phases and are expected to serve, not only for the detection of potential $0 \nu \beta \beta$ decay events, but also for the direct detection of neutrinos through inverse ' $\beta$ decay' by the capture of neutrinos. Another facet of neutrino detection lies in the excitation of $1^{+}$states by neutrinos [59]. Hence, the $M 1$ response of relevant nuclei plays an important role in the characterization of such detectors and also in rate estimates using nuclear models such as the quasiparticle random-phase approximation.

The beam requirements for carrying out the nuclear structure research using NRF described in this section are given in table 1.

3.1.2. Photon-induced nuclear fission. Nuclear fission is a highly exothermic and strongly collective nu clear process in which most of the energy is released through the kinetic energy of the ejected fission fragments. The evolution of a fissioning system proceeds from the initial impact of the incident particle through the intermediate saddle point(s), then through scission, and finally to the configuration of separated fission fragments. This evolution is governed by a multi-dimensional potential-energy surface (PES) and by the shell structure of the fragments. Development of reliable theoretical models of nuclear fission is important for basic research and for the development of nuclear energy and nuclear security technologies. For these purposes, data for different types of observables are needed, including fission product yields over wide ranges of masses and half lives, as well as the kinetic energy and angular distributions of the emitted fragments and neutrons. 
To first approximation, the fission barrier of a heavy nucleus can be studied by measuring the fission probability as a function of the excitation energy. By comparing the experimentally determined fission probabilities to the results obtained with the WKB approximation, the shape of the fission barrier (its height and curvature parameter) can be determined.

Significant progress has been made in studying the multiple-humped fission barrier landscape of actinides, where a strongly deformed deep third minimum in the potential landscape was established $[60,61]$. This is illustrated in figure 7 , where horizontal dashed lines show transmission resonances in the superdeformed (SD) second minimum and in the hyperdeformed (HD) third minimum. One expects that the HD minimum in a cluster description consists of a rather spherical ${ }^{132} \mathrm{Sn}$-like component with magic neutron and proton numbers $N=82$ and $Z=50$. Recent theoretical results $[62,63]$ highlight the role of shell corrections in the prominence of the third minima for thorium and light uranium isotopes. There is a clear trend suggesting that lower $N$ values produce larger neutron shell corrections and, thus, more prominent third minima. This is in stark disagreement with experimental results. Using the ${ }^{231} \mathrm{~Pa}\left({ }^{3} \mathrm{He}, d \mathrm{f}\right)$ reaction, Csige et al [64] studied sub-barrier fission in ${ }^{232} \mathrm{U}$ and inferred a fission barrier with a well-formed third minimum that does not agree with the theoretical predictions. Pronounced third minima have also been inferred to exist through observation of fine structure in the cross section of transfer-reaction-induced fission or through sub-barrier photofission cross-section measurements. There is particular interest in identifying resonances in the third minimum, where very large deformations cause the GDR to split into two components with a low-lying oscillation along the long symmetry axis with a typical excitation energy of $4-5 \mathrm{MeV}$. It is expected that these resonances may have a significantly enhanced $\gamma$-ray width $\Gamma_{\gamma}$ of about $100 \mathrm{eV}$ in the population of the third minimum, while, for the second minimum, one expects a $\gamma$-ray width of only about $1 \mathrm{eV}$. $E 1$ resonances excited from the ground state will have about the same absolute excitation strength as resonances in the third minimum, but resonances in the third minimum will be much broader, with a total width of about $1 \mathrm{keV}$.

Photofission measurements enable the selective investigation of extremely deformed nuclear states in the light actinides and can be utilized to better understand the landscape of the multiple-humped PES of these nuclei. The selectivity of these measurements stems from the low and reasonably well-defined amount of angular momentum transferred during the photoabsorption process. High-resolution studies can be performed on the mass, atomic number, and kinetic energy distributions of the fission fragments following the decay of well-defined initial states in the first, second and third minima of the PES in the region of the light actinides. The beams available at the next-generation laser Compton source facilities will enable high fidelity studies of the PES using transmission resonance spectroscopy, as well as studies of heavy clusterization in the actinides, and of rare fission processes such as ternary fission. Each of these opportunities is discussed in this section.

3.1.2.1. Transmission resonance spectroscopy in photofission. The approach to investigating extremely deformed collective and single-particle nuclear states of the light actinides is based on the observation of transmission resonances in the prompt fission cross section. Observing transmission resonances as a function of the excitation energy allows the identification of the excitation energies of the SD and HD states. Moreover, the observed states can be ordered into rotational bands, with moments of inertia proving that the underlying nuclear shape of these states is indeed an SD or an HD configuration. For the identification of the rotational bands, the spin information can be obtained by measuring the angular distributions of the fission fragments. Furthermore, the PES of the actinides can be parameterized very precisely by analyzing the overall structure of the fission cross section, and by fitting it with the results of nuclear reaction code calculations. 

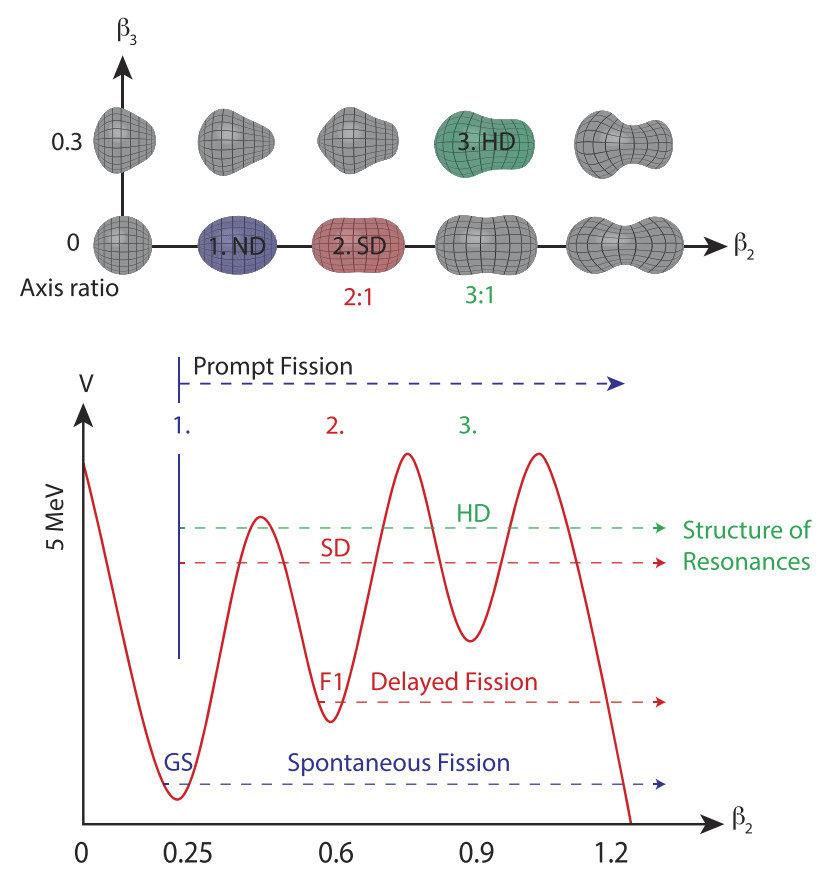

Figure 7. Schematic overview of the multiple-humped fission barrier in actinide isotopes, along with the corresponding nuclear shapes. The lower part shows a cut through the potential energy surface along the fission path, revealing an SD second minimum at an axis ratio of 2:1 and an HD third minimum at an axis ratio of 3:1. This figure, including the energies and locations of the saddle points and minima were reprinted from [60], Copyright (2002), with permission from Elsevier. In the upper part, the corresponding nuclear shapes are displayed as a function of the quadrupole and octupole degrees of freedom.

So far, transmission resonances in the light actinides have been studied primarily in lightparticle-induced nuclear reactions. These studies do not benefit from the same selectivity found in photonuclear excitation and, consequently, they are complicated by a statistical population of the states in the second (and third) minimum. These measurements have also suffered from a dominant prompt-fission background. By contrast, the next-generation laser Compton $\gamma$-ray sources will enable the identification of sub-barrier transmission resonances with very low integrated cross sections down to $\Gamma \sigma \approx 0.1 \mathrm{eVb}$ and in so far unexamined nuclei. The narrow energy bandwidth of the next generation $\gamma$-ray sources will enable a significant reduction of the presently dominant background from non-resonant processes.

Besides exploring the level structure in the second and third minima of the fission barrier of the light actinides, the harmonicity of the potential barrier can also be examined, and the parameters of the fission barrier can be extracted. Such fission barrier parameters are crucial inputs for cross-section calculations in the thorium-uranium fuel cycle of fourth-generation nu clear power plants. The selectivity of the photofission measurements allows high-resolution investigation of fission resonances in photofission in the second and third minima of the fission barrier. Detailed studies of SD and HD states via transmission resonance spectroscopy is relevant also for achieving much cleaner energy production by an efficient transmutation of the long-lived, and most hazardous radioactive components of nuclear waste, and by controlling the fission process through using entrance channels via HD states. 
Table 2. Suggested beam parameters for the photofission research program.

\begin{tabular}{lc}
\hline Parameter & Value \\
\hline Energy & $5-20 \mathrm{MeV}$ \\
Flux $(\gamma / \mathrm{s})$ & $10^{9}$ at $3 \%$ FWHM \\
Polarization & Linear and circular \\
Diameter & $10 \mathrm{~mm}$ on target \\
Beam repetition rate & Few MHz \\
Beam pulse width & $<1.0 \mathrm{~ns}$ \\
Macropulse width & $1 \mu$ s to $10 \mathrm{~s}$ \\
Macropulse repetition rate & $0.1 \mathrm{~Hz}$ to $500 \mathrm{kHz}$ \\
\hline
\end{tabular}

3.1.2.2. Photofission as a probe of heavy clusterization in the actinides. Theoretical considerations suggest that in a cluster description, the HD configuration of a light actinide consists of a spherical ${ }^{132} \mathrm{Sn}$-like component with magic neutron and proton numbers $N=82$ and $Z=50$, respectively, complemented by an attached, elongated second cluster of nucleons. Since the fission-product mass distribution is distinctly determined by the configuration at the scission point, and the third minimum is very close to the scission configuration, it is expected that the mass distribution, following the decay of an HD nucleus, will exhibit a pronounced asymmetric structure. However, such a dramatic effect of the shell structure has not been observed so far due to the fact that available studies used particle-induced fission.

Brilliant quasi-monoenergetic $\gamma$-ray beams will enable high-resolution investigations of the mass, atomic number, and kinetic energy distributions of the fission fragments following the decay of states in the first, second and third minima of the PES in the region of the light actinides. In these measurements, the heavy clusterization and the predicted cold valleys of the fission potential can be studied for the first time. Data on the heavy cluster formation will provide valuable information on the fission dynamics.

3.1.2.3. Investigation of rare fission modes: ternary photofission. So far, information on ternary and more exotic fission modes has been deduced from neutron-induced and spontaneous fission experiments. Ternary particles are released very close to the scission point, thus providing valuable information on both the scission-point configuration of the fissioning nucleus and the dynamics of the fission process. However, ternary photofission has never been studied due to the very low cross section of the reaction channel.

In such studies, the geometry can be fixed by using polarized $\gamma$-ray beams, which is a clear advantage over neutron-induced or spontaneous fission experiments. Moreover, by employing quasi-monoenergetic $\gamma$-ray beams, excitation-energy correlations of the ternary fission process can be explored with good resolution. These experiments investigate open problems such as the mechanism of ternary particle emission, the role of the deformation energy and of the spectroscopic factor, and the possible formation of heavier clusters. It will be interesting to measure light-particle decay in photofission and to search for the predicted enhanced $\alpha$ decay of SD and HD states of the light actinides.

The availability of brilliant quasi-monoenergetic $\gamma$-ray beams will make it possible to study the angular distribution of the ternary particles. These, in turn, will provide important spectroscopic information on the fissioning system. The beam requirements to carry out the photofission research program described in this section are given in table 2. 
Table 3. Suggested beam parameters for the nuclear astrophysics program. The beam parameters required to carryout the ${ }^{16} \mathrm{O}(\gamma, \alpha){ }^{12} \mathrm{C}$ measurements are the most demanding in this research area. In addition to the listed beam attributes, another important requirement is that the beam on target have a very small bremsstrahlung component, in order to minimize backgrounds caused by $\gamma$-ray-induced particle production.

\begin{tabular}{lc}
\hline Parameter & Value \\
\hline Energy & $2-20 \mathrm{MeV}$ \\
Flux $(\gamma / \mathrm{s})$ & $10^{11}$ at $1 \% \mathrm{FWHM}$ \\
Polarization & Linear and circular \\
Diameter & $10 \mathrm{~mm}$ on target \\
Beam repetition rate & Few MHz \\
Beam pulse width & $<1.0 \mathrm{~ns}$ \\
\hline
\end{tabular}

\subsection{Nuclear astrophysics}

Many questions in astrophysics require a detailed understanding of stars and stellar properties, thus challenging stellar models to become more sophisticated, quantitative, and realistic in their predictive power. This in turn requires more detailed input, such as thermonuclear reaction rates and opacities, and a concerted effort to validate models through systematic observations. Consequently, the study of nuclear reactions in the Universe remains at the forefront of nuclear physics and astrophysics research.

Nuclear reactions generate the energy in stars and are responsible for the synthesis of the elements. When stars eject part of their matter through various means, they enrich the interstellar medium with their nuclear ashes and thereby provide the building blocks for the birth of new stars, of planets, and of life itself. Element synthesis and nuclear energy generation in stars are the two primary research topics in nuclear astrophysics. Both require accurate knowledge of charged-particle- and neutron-induced nuclear reactions that take place in the hot stellar plasma. It is remarkable how the quantum mechanical nature of atomic nuclei influence the macroscopic properties of stars.

The DOE report The 2015 Long Range Plan for Nuclear Science: Reaching for the Horizon organizes nuclear astrophysics into five broad topical areas: (1) the origin of the elements, (2) the life of stars, (3) the death of stars, (4) the matter of neutron stars, and (5) connections: dark matter, QCD phase diagram, weak interactions and neutrinos. The beams available at the nextgeneration laser Compton $\gamma$-ray source facilities will enable measurements that contribute to the first four topical areas. The main opportunities are for cross-section measurements of $\left(\gamma, \gamma^{\prime}\right)$ NRF processes and ( $\gamma$, particle) reactions. The NRF measurements provide important information for determining PSFs, $\gamma$-ray transition probabilities, and nuclear structure spectroscopic information, all of which are inputs to nuclear astrophysics reaction-network calculations. The $(\gamma$, particle) reaction measurements provide data that are important input for $\gamma$-ray-induced reactions on stable nuclei in stars and also for the time reverse of particle capture on unstable nuclei. These measurements are particularly relevant for p-process, s-process, and r-process nucleosynthesis. The most important contribution to nuclear astrophysics that will come from opportunities created by the beam capabilities of a next-generation laser Compton $\gamma$-ray source is the measurement of the ${ }^{16} \mathrm{O}(\gamma, \alpha){ }^{12} \mathrm{C}$ reaction cross section as a means for determining the cross section for the ${ }^{12} \mathrm{C}(\alpha, \gamma){ }^{16} \mathrm{O}$ reaction at center-of-mass energies important for carbon burning in massive stars using time reversal invariance. Some examples of research opportunities at the next-generation laser Compton $\gamma$-ray source are outlined below. Table 3 gives the suggested beam parameters for the nuclear astrophysics program. 
3.2.1. The origin of the elements: p-process and s-process nucleosynthesis. Models of pprocess and s-process nucleosynthesis require reliable $(\gamma, n),(\gamma, p)$ and $(\gamma, \alpha)$ reaction cross sections on hundreds of stable and unstable nuclei. The proton-rich nuclei cannot be produced by neutron capture reactions. Complete network calculations on p-process nucleosynthesis include several hundred isotopes and the corresponding reaction rates. Theoretical predictions of the rates, normally in the framework of the Hauser-Feshbach (HF) theory, are necessary for modeling the nucleosynthesis reaction network. The reliability of these calculations should be tested experimentally, especially at rate-limiting paths in the network. Different approaches are available and necessary to improve the experimental data base for the p-process. The $(\gamma, n)$ cross sections in the energy regime of the GDR have already been measured extensively (see, e.g., [65]). More recently, substantial effort has be devoted to using beams with a continuous bremsstrahlung spectrum to determine the reaction rates without any assumptions on the shape of the cross section's energy dependence in the astrophysically relevant energy region, close to the reaction threshold [66-68]. A determination of the reaction rates by an absolute cross section measurement is also possible using monoenergetic photon beams produced by a laser Compton $\gamma$-ray source [69]. The beam capabilities at next-generation laser Compton $\gamma$-ray sources will open the possibility of higher accuracy $(\gamma, n)$ cross-section measurements and measurements on nuclei with low natural abundances, for which the amount of target material will be small.

In contrast, the experimental knowledge about the $(\gamma, p)$ and $(\gamma, \alpha)$ reactions in the corresponding Gamow window is smaller. In fact, the experimental data is based on the observation of the time reversal $(p, \gamma)$ and $(\alpha, \gamma)$ cross sections, respectively [70-74] for the proton-rich nuclei with mass numbers around 100. Due to the difficulties concerning the experimental accessibility of the $(\gamma, \alpha)$ reaction rates, a method using elastic $\alpha$ scattering has been established $[75,76]$. It would be a tremendous improvement in the quality of the database to measure these rates directly using photon beams. Having a significant impact on this database will require a program of systematic measurements on a broad range of nuclei. This type of program would be made possible by a next-generation laser Compton $\gamma$-ray source.

The heavy elements above the so-called iron peak are mainly produced in neutron capture processes: the $r$ process (r: rapid neutron capture) deals with high neutron densities, well above $10^{20} \mathrm{~cm}^{-3}$, and temperatures of around 2 to $3 \mathrm{GK}$. It is thought to occur in explosive scenarios such as supernovae [78, 79] and was recently verified in neutron star merger via gravitational wave observations [80]. In contrast, the average neutron densities during s-process nucleosynthesis ( $\mathrm{s}$ : slow neutron capture) are rather small (around $10^{8} \mathrm{~cm}^{-3}$ ), so that the neutron capture rate $\lambda_{n}$ is normally well below the $\beta$-decay rate $\lambda_{\beta}$, and the reaction path is therefore close to the valley of $\beta$ stability [81-83]. However, during the peak neutron densities, branching occurs at unstable isotopes with half-lives as low as several days. The half-lives of these branch points are normally known with high accuracy, at least under laboratory conditions, and rely on theory only for the extrapolation to stellar temperatures [84]. However, their neutron capture cross sections are accessible to direct experiments only in special cases. For this reason, nuclear astrophysics reaction-network simulations currently rely heavily on HF model calculations of the critical neutron capture cross sections. The difficulty is that there are many examples where the results of HF model calculations differ substantially from measurements (see, e.g., reference [85]). Thus, experimental constraints on the theoretical predictions of these branch points are needed. Laser Compton $\gamma$-ray sources enable measurements of the inverse $(\gamma, n)$ reaction, which can also provide information about the stellar enhancement factors (SEFs). The SEF accounts for the difference in the neutron-capture cross section measured in the laboratory and the effective cross section in the stellar environment. Because nuclei in stars spend much of the time in excited states, capture reactions mostly occur on excited states, not the ground state. 


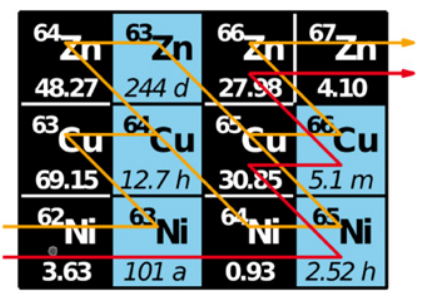

Figure 8. The s-process reaction path in the $\mathrm{Ni}-\mathrm{Cu}-\mathrm{Zn}$ region. During $\mathrm{He}$-core burning (yellow), the temperature and neutron density are low compared to those during C-shell burning (red). Therefore, the unstable nucleus ${ }^{63} \mathrm{Ni}$ either experiences decay or radiative neutron capture and acts as a branch-point nucleus. The isotopic abundance pattern of copper and zinc depends strongly on the stellar neutron-capture rate of ${ }^{63} \mathrm{Ni}$. Reprinted figure with permission from [77], Copyright (2013) by the American Physical Society.

In addition to determining the cross sections and the SEFs directly from $(\gamma, n)$ cross-section measurements, the SEFs can be calculated using PSFs determined from NRF measurements. The importance of each nucleus and reaction in the s-process network must be analyzed on its own merits. An example of the type of information that can be obtained using linearly polarized mono-energetic photon beams is illustrated in the proposed NRF measurement on ${ }^{64} \mathrm{Ni}$ at $\mathrm{HI} \gamma \mathrm{S}$.

The ${ }^{64} \mathrm{Ni}$ nucleus is the product of neutron capture on ${ }^{63} \mathrm{Ni}$, which is a branch-point nucleus. Figure 8 illustrates the situation during He-core burning (yellow) and C-shell burning (red) of a massive star, where the weak component of the s-process originates [86]. As the branching is very sensitive to the ${ }^{63} \mathrm{Ni}(n, \gamma)$ cross section, a measurement was performed at CERN using a radioactive ${ }^{63} \mathrm{Ni}$ target $[77,87]$. However, the reaction rate in the hot environment of the stellar plasma differs significantly from the measured value because excited states are populated [88]. In the case of ${ }^{63 \mathrm{gs}} \mathrm{Ni}(n, \gamma)$, the stellar rate is still around $90 \%$ at He-core burning temperatures, but it drops to around $40 \%$ at the higher temperature in the C-shell burning phase [77]. HF model calculations are required to account for the stellar enhancement [89]. These calculations rely on the PSF in ${ }^{64} \mathrm{Ni}$, which can be deduced from photoabsorption cross sections and the decay properties of low-spin states. The linearly polarized monoenergetic beams available at laser Compton $\gamma$-ray sources enable model-independent determination of photoabsorption cross sections as a function of the excitation energy, and consequently, a determination of the PSFs (see, e.g., [30, 33, 34, 90, 91]).

3.2.2. The life of stars: the ${ }^{12} \mathrm{C}(\alpha, \gamma)$ reaction cross section. Late-stage red giant stars produce energy in their interiors via helium burning. In first generation stars, helium burning proceeds through the $3 \alpha$ process and then ${ }^{12} \mathrm{C}(\alpha, \gamma){ }^{16} \mathrm{O}$, while in later generation stars, $\alpha$ captures also involve the various $\mathrm{CNO}$ seed nuclei. In both cases, the ${ }^{12} \mathrm{C}(\alpha, \gamma){ }^{16} \mathrm{O}$ reaction helps to regulate the efficiency of helium burning in massive stars (those with masses greater than the suns). It also determines core mass, temperature and density during the latter stages of stellar evolution, and ultimately the mass of the iron core in the incipient supernova. In addition, the carbon-tooxygen ratio $(\mathrm{C} / \mathrm{O})$ influences the abundances of elements produced in the ensuing explosion. After several decades of effort, the uncertainty in the rate of the $\alpha$-particle capture reaction on ${ }^{12} \mathrm{C}$ is still large enough to substantially limit our understanding of the latter stages of stellar evolution. To resolve this problem, the astrophysical $S$ factor for the ${ }^{12} \mathrm{C}(\alpha, \gamma){ }^{16} \mathrm{O}$ reaction must be determined to an accuracy better than about $10 \%$ in the Gamow window $\left(E_{\mathrm{cm}}=300 \mathrm{keV}\right)$, which is indicated by the vertical band in figure $9[92,93]$. 


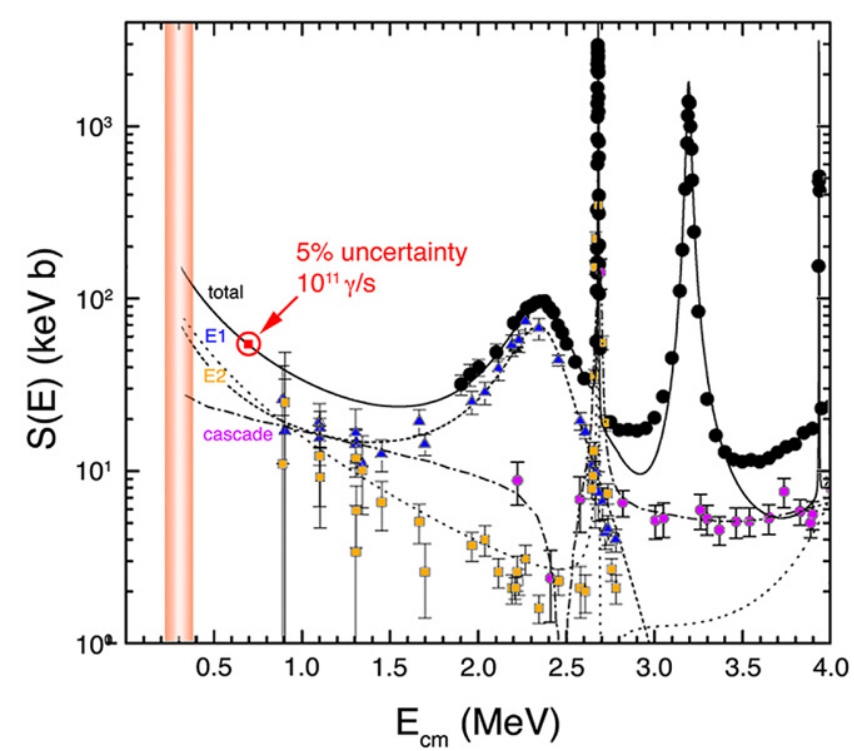

Figure 9. Plot of total $S$-factor data (filled-in circles) [94] for ${ }^{12} \mathrm{C}(\alpha, \gamma){ }^{16} \mathrm{O}$ compared with $E 1$ (open triangles) and $E 2$ (open squares) $\gamma$-ray measurements [95] and the $E_{x}=$ $6.05 \mathrm{MeV}$ cascade data (open circles) [96]. The solid line represents the sum of the single amplitudes of an $R$-matrix fit [97], while the dotted and dashed lines are the $E 1$ and $E 2$ amplitudes, respectively. In addition, the $R$-matrix fit of [96] to their cascade data is shown as the dot-dashed line. The latter component is not included in the sum and might explain the high yield in the $S$-factor data between the resonances. Reproduced from [98]. ๑ IOP Publishing Ltd. All rights reserved.

As shown in figure 9, the cross section has been measured at various levels of precision down to a center-of-mass $(\mathrm{cm})$ energy of $1.2 \mathrm{MeV}$; then it must be extrapolated down to $300 \mathrm{keV}$, the energy needed for stellar reaction-rate calculations. One of the major uncertainties in performing the extrapolation arises from the presence of several resonances which contribute to the cross section at energies around $E_{\mathrm{cm}}=0.75 \mathrm{MeV}$. Above $E_{\mathrm{cm}}=1 \mathrm{MeV}$, the elastic scattering and capture reactions are dominated by a broad $1^{-}$resonance at an excitation energy in ${ }^{16} \mathrm{O}$ of $E_{x}=9.59 \mathrm{MeV}\left(E_{\mathrm{cm}}=2.43 \mathrm{MeV}\right)$ and a narrow $2^{+}$state at $E_{x}=$ $9.85 \mathrm{MeV}\left(E_{\mathrm{cm}}=2.70 \mathrm{MeV}\right)$. However, a $1^{-}$state at $E_{x}=7.12 \mathrm{MeV}$, just $42 \mathrm{keV}$ below threshold, determines the capture cross section in the astrophysically relevant energy region, including its interference with the higher lying $1^{-}$and $2^{+}$states. In addition, broad high-lying states and direct processes produce a coherent background that affects the energy dependence of the cross section and thereby the extrapolation.

Direct measurements of the ${ }^{12} \mathrm{C}(\alpha, \gamma){ }^{16} \mathrm{O}$ cross section at energies below $E_{\mathrm{cm}}=2 \mathrm{MeV}$ have been attempted for over 30 years. The major difficulty encountered in these experiments is the intense neutron background arising from the ${ }^{13} \mathrm{C}(\alpha, n)$ reaction. This background tends to swamp the $\gamma$-ray detector. The $\gamma$-ray beams produced by laser Compton sources have a narrow energy spread and thus offer an alternative technique for measuring the cross section for $\alpha$ capture on ${ }^{12} \mathrm{C}$ at energies important to astrophysics as discussed above. The principle of detailed balance allows the determination of the $(\alpha, \gamma)$ cross section from the measurement of the cross section for the time reversed $(\gamma, \alpha)$ reaction. An added advantage of using the $(\gamma, \alpha)$ reaction over the direct reaction is that the cross section in the gamma window is enhanced by 


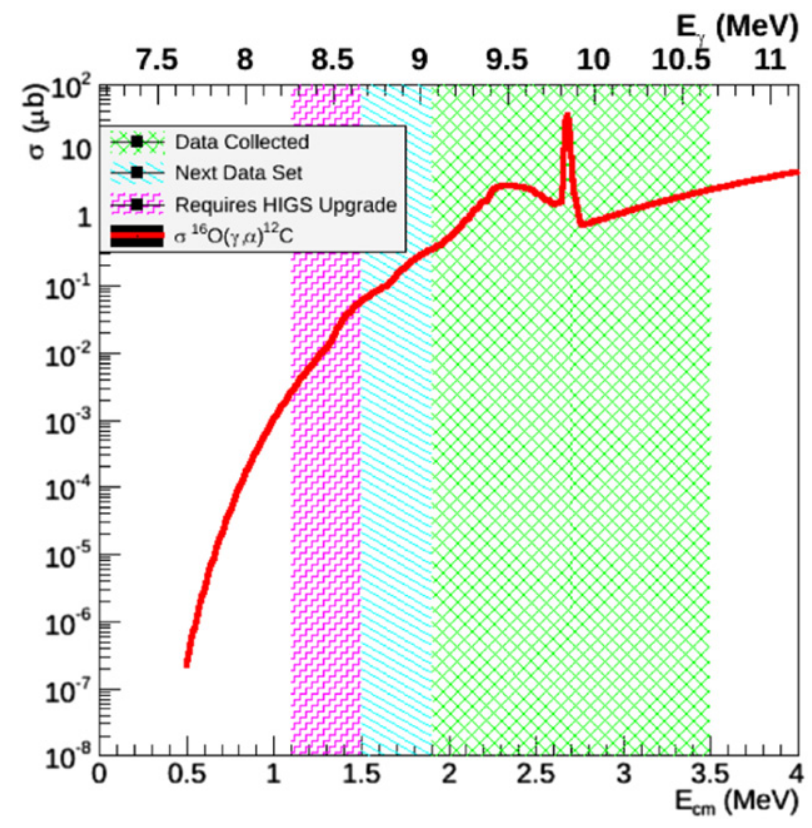

Figure 10. Plot of the cross section of the ${ }^{16} \mathrm{O}(\gamma, \alpha)^{12} \mathrm{C}$ reaction as a function the system center-of-mass energy (bottom axis) and the incident $\gamma$-ray beam energy (top axis). The green hash shows the region where data have been collected at $\mathrm{HI} \gamma \mathrm{S}$ using the optical time-projection chamber (TPC). The blue filled bar is the lowest energy region experimentally accessible at $\mathrm{HI} \gamma \mathrm{S}$ using current target and detector technologies. The energy region indicated by the red brick filled area is what would be possible with an intensity upgrade of $\mathrm{HI} \gamma \mathrm{S}$.

about a factor of 50 due to detailed balance. This experimental concept has been demonstrated at $\mathrm{HI} \gamma \mathrm{S}$ using a $\mathrm{CO}_{2}$ gas-filled optical TPC [99, 100]. The calculated cross section for the ${ }^{16} \mathrm{O}(\gamma, \alpha){ }^{12}$ reaction is shown in figure 10 . An upgrade of $\mathrm{HI} \gamma \mathrm{S}$ to increase the $\gamma$-ray beam flux on target by a factor of about fifty in the energy region important for this reaction, would enable meaningful measurements in the red shaded energy range $\left(\left\langle E_{\mathrm{cm}}\right\rangle=1.3 \mathrm{MeV}\right)$ in the figure. The next-generation laser Compton sources could potentially deliver more than an additional factor of 500 in beam flux on target relative to $\mathrm{HI} \gamma \mathrm{S}$, thereby enabling measurements down to about $\left\langle E_{\mathrm{cm}}\right\rangle=700 \mathrm{keV}$. This measurement should be performed at next-generation laser Compton $\gamma$-ray source facilities using a variety of experimental techniques, such as gas TPCs (both optical and charge readout), silicon strip detectors with thin targets, and total cross-section measurements using super-heated high-purity water detectors (e.g., as in reference [101]).

3.2.3. The death of stars: r-process nucleosynthesis. More than half of the heavy nuclei with $A>120$ are produced by $\mathrm{r}$-process nucleosynthesis. The r-process involves nuclear reactions driven by rapid neutron capture, where the neutron capture rate is faster than the competing beta decay. The likely environments for the r-process are type-II supernovae and merging neutron stars. Simulations of r-process nucleosynthesis require reliable $(n, \gamma)$ reaction cross sections on hundreds of stable and unstable nuclei. Theoretical predictions of the rates, normally in the framework of HF theory, are necessary for modeling neutron capture on unstable nuclei. The reliability of these calculations should be tested experimentally, especially at rate-limiting paths in the network. The $(\gamma, n)$ time-reversed photodisintegration reaction offers a mechanism 


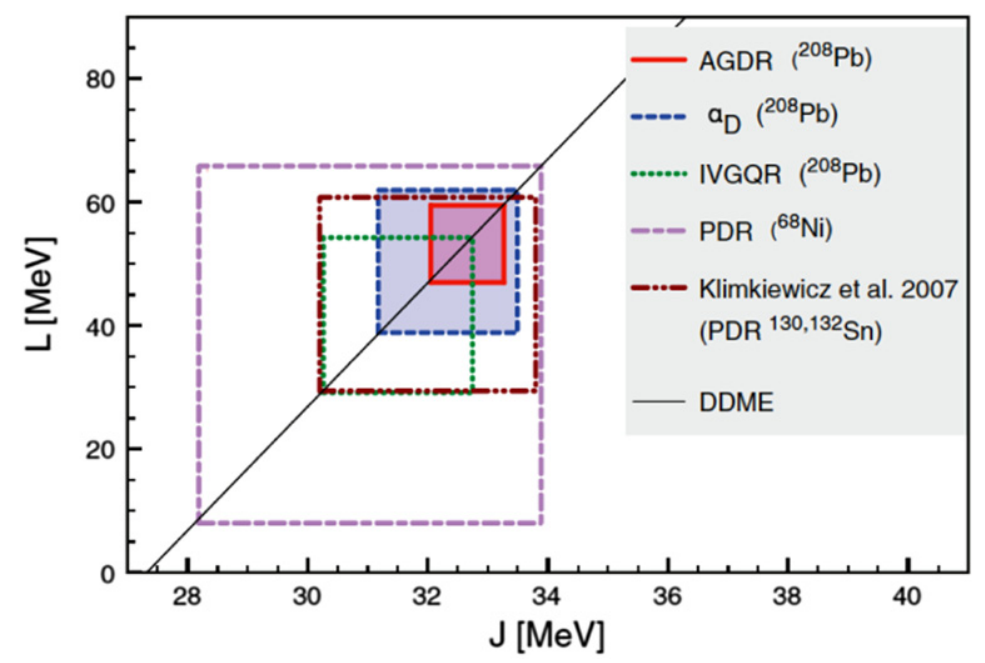

Figure 11. Constraints of the symmetry energy at saturation $J$ and slope parameter $L$, obtained from a comparison of relativistic nuclear energy density functional results and data on anti-analog giant dipole resonance [109] and IVGQR [104] excitation energies in ${ }^{208} \mathrm{~Pb}$; the dipole polarizability $\alpha \mathrm{D}$ of ${ }^{208} \mathrm{~Pb}$ [110]; and the PDR energy-weighted strength in ${ }^{68} \mathrm{Ni}$ [111] and ${ }^{130,132} \mathrm{Sn}$ [107]. Reprinted figure with permission from [112], Copyright (2014) by the American Physical Society.

for determining the neutron capture cross section and level density of radioactive nuclei. Nextgeneration laser Compton $\gamma$-ray sources will enable measurements on isotopes with low natural abundances and thus very limited sample sizes.

3.2.4. Neutron stars: the EOS of neutron-rich matter. Obtaining information about the detailed structure of the crust of a neutron star is an important open challenge in astrophysics. The crust is composed of non-uniform neutron-rich solid matter that is about $1 \mathrm{~km}$ thick and located above a liquid core $[102,103]$. The inner crust comprises the region from the density at which neutrons drip from nuclei to the inner edge separating the solid crust from the homogeneous liquid core. While the density at which neutrons drip from nuclei is well determined, the transition density at the inner edge is much less certain because of insufficient knowledge of the EOS of neutron-rich nuclear matter. Measurements of collective responses of neutronrich nuclei provide constraints on the nuclear EOS. The monoenergetic and linearly polarized beams at laser Compton $\gamma$-ray sources enable unique measurements of dipole and quadrupole excitations. Those most relevant to exploring the EOS are studies of the PDR on nuclei at the neutron rich end of an isotope chain using NRF and determination of the centroid energy and width of the isovector giant quadrupole resonance (IVGQR) via Compton scattering [104]. The underlying structure of the PDR is often interpreted as an oscillation of less bound valence neutrons forming a neutron skin $[105,106]$. However, the true nature of the PDR is a matter of ongoing discussion. Furthermore the PDR and the $E 1$ strength in general have a direct connection to the neutron skin of nuclei and the symmetry energy of nuclear matter [107, 108] (figure 11). 


\subsection{Hadronic parity violation}

The capabilities of a next generation laser Compton $\gamma$-ray source, combined with current and expected advances in theory and lattice calculations, provide the opportunity to make significant progress in our understanding of HPV. PV in nuclear processes can probe the SM and point toward possible SM extensions. In the SM sector, HPV provides an important probe of two phenomena that are not well understood: neutral-current nonleptonic weak interactions and nonperturbative strong dynamics. While PV is well understood in quark-quark weak interactions, it is ultimately the interplay of different forces at different length scales in the SM that is responsible for HPV phenomena. Neutral-current interactions are suppressed in flavorchanging hadronic decays, making HPV between nucleons the best place to study neutralcurrent effects. Because parity violating NN interactions are the manifestation of the interplay of nonperturbative strong effects and the short-range weak interactions between quarks, they are sensitive to short-distance quark-quark correlations inside the nucleon. Quark-quark and $\mathrm{NN}$ weak interactions also induce parity-odd effects in electron scattering [113-116], nuclear decays [117], compound nuclear resonances [118, 119], and atomic structure, where they are the microscopic source for nuclear anapole moments [120-123]. The comparison between NN weak amplitudes in few-nucleon systems and heavy nuclei can also test the statistical theory of PV in compound nuclei, which will become useful in the future for the interpretation of time reversal violation experiments in the transmission of polarized neutrons through polarized nuclear targets on p-wave compound nuclear resonances [124].

The NN weak interactions are therefore worth understanding both for their own sake and for other areas of nuclear, particle, and atomic physics. Agreement between theory and experiment in this area pushes the 'complexity' frontier of the SM, as it requires a quantitative understanding of strong, electromagnetic, and weak interactions in the strongly-interacting regime of QCD all acting in the same system. This research program will also stretch the technology of lattice gauge theory calculations [125] to its computational limits.

This section explores possibilities for measuring HPV in few nucleon-systems and in the mixing of parity doublet states in light nuclei using circularly polarized high-intensity photon beams.

3.3.1. Hadronic parity violation in few-nucleon systems. Weak NN amplitudes at low energy are suppressed by six to seven orders of magnitude compared to strong NN amplitudes and are therefore difficult to observe. While these effects can be amplified by several orders of magnitude by nuclear dynamics in complex systems, the unambiguous extraction of the weak interactions among nucleons requires parity violating measurements in very light nuclei, where theory is under good control. At the moment this includes the deuteron, the triton, and ${ }^{3} \mathrm{He}$, with the four-nucleon system in the pipeline. These very light systems can be calculated using two- and three-nucleon interactions, which in turn are understood in terms of effective field theories (EFTs) that systematically incorporate the symmetries of QCD. The EFT approach can consistently be applied to strong and weak nucleon interactions as well as to external currents. There is also growing optimism that parity-odd effects in $A>4$ nuclei can be calculated. We are at a juncture in the development of theory where PV may soon be understood in terms of QCD dynamics even in many body nuclei. Ongoing programs [126] are pursuing the matching of LQCD to EFTs, which are used as input into many-body calculations of heavier nuclei. Both SM and beyond-the-SM physics can be treated in this way.

At leading order in the EFT power counting, and at very low photon energies (below $10 \mathrm{MeV}$ ), there are five parity violating low-energy constants (LECs) that parameterize the 
short-distance physics [127-129]. ${ }^{35}$ The values of these LECs cannot be determined within the EFT framework but have to be extracted from experiment or ultimately from calculations involving nonperturbative QCD. Recent work shows that upon combining the EFT approach with the large- $N_{\mathrm{c}}$ expansion of QCD, the number of independent parity-violating LECs reduces to two at leading order [134]. One of these is the $\Delta I=2$ isotensor coupling, which is poorly constrained from existing measurements. Combining the existing data for $\vec{p}+p$ scattering (which constrains the remaining leading-order isoscalar piece) with the results of a measurement of the $\Delta I=2$ contribution would therefore constrain all terms at leading order in this combined EFT and large- $N_{\mathrm{c}}$ expansion [135].

An important experimental constraint on the isovector parity violating interactions comes from the parity violating asymmetry from polarized neutron capture on the proton, $\vec{n}+p \rightarrow$ $D+\gamma$, recently measured by the NPDGamma collaboration at the Spallation Neutron Source (SPS) at ORNL [136]. In the next few years we expect to possess two additional pieces of experimental information on the NN weak interaction in few-nucleon systems. The analysis of PV in $\vec{n}+{ }^{3} \mathrm{He} \rightarrow{ }^{3} \mathrm{H}+p$ completed recently at the SPS at ORNL will be published, and another experiment on PV in $\vec{n}+{ }^{4} \mathrm{He}$ spin rotation [137] will be performed at the new NG-C neutron beam line at the NIST Center for Neutron Research (NCNR). However, these will yield scant experimental information on the elusive $\Delta I=2$ component of the $\mathrm{NN}$ weak interaction. Fortunately the $\Delta I=2$ operator is the easiest target for a quantitative lattice calculation from the SM because it does not receive contributions from disconnected diagrams. The isotensor operator is scheduled to be calculated on the lattice in the next few years [138, 139]. Lattice constraints on other $\mathrm{NN}$ weak amplitudes are many years out, at least at physical quark masses.

The cleanest experimental channel for extracting the $\Delta I=2$ component in few-body $\mathrm{PV}$ is P-odd deuteron photodisintegration near threshold using the helicity-dependent photodisintegration cross section for circularly polarized photons. Limits from previous attempts exist from Chalk River [140] and from the reverse reaction [141], but did not resolve a nonzero value. Experiments to measure this observable have been proposed in the past at JLab [142, 143], SPRING-8, and the Shanghai Synchrotron, but they have not yet been realized. It was therefore listed in the last NSAC long-range plan as a priority NN weak interaction measurement for the future. In addition, it is possible to contemplate other PV experiments involving helicitydependent reactions with circularly polarized photons in few-nucleon systems, such as the breakup of three-nucleon systems. We emphasize the importance of a $\vec{\gamma} d \rightarrow p n$ parity-violation measurement because of the unique information it will provide about the weak interaction in hadronic systems and the state of EFT and lattice calculations.

The parity-violating asymmetry in deuteron photodisintegration, including its energy dependence, has been considered in pionless EFT in references [144, 145]. The next-to-leading order results of reference [145] were combined with different model estimates for the LECs to determine the expected size of the parity-violating asymmetry $A_{L}^{\gamma}$, to estimate a figure of merit (FOM), and to extract the best energy regime for performing the experiment. $A_{L}^{\gamma}$ is computed as the ratio of the difference in the photodisintegration cross section measured with positive $(h=+1)$ and negative $(h=-1)$ beam helicity divided by the unpolarized cross section, which is the sum of the positive and negative helicity cross sections. The beam helicity is $h=+1$ when the polarization vector of the circularly polarized beam is in the direction of the beam momentum vector. $A_{L}^{\gamma}$ for the photodisintegration of the deuteron computed using various

\footnotetext{
${ }^{35}$ At higher energies, pions have to be treated as active degrees of freedom and this increases the number of LECs. PV in chiral EFT has been considered recently in references [130-133].
} 


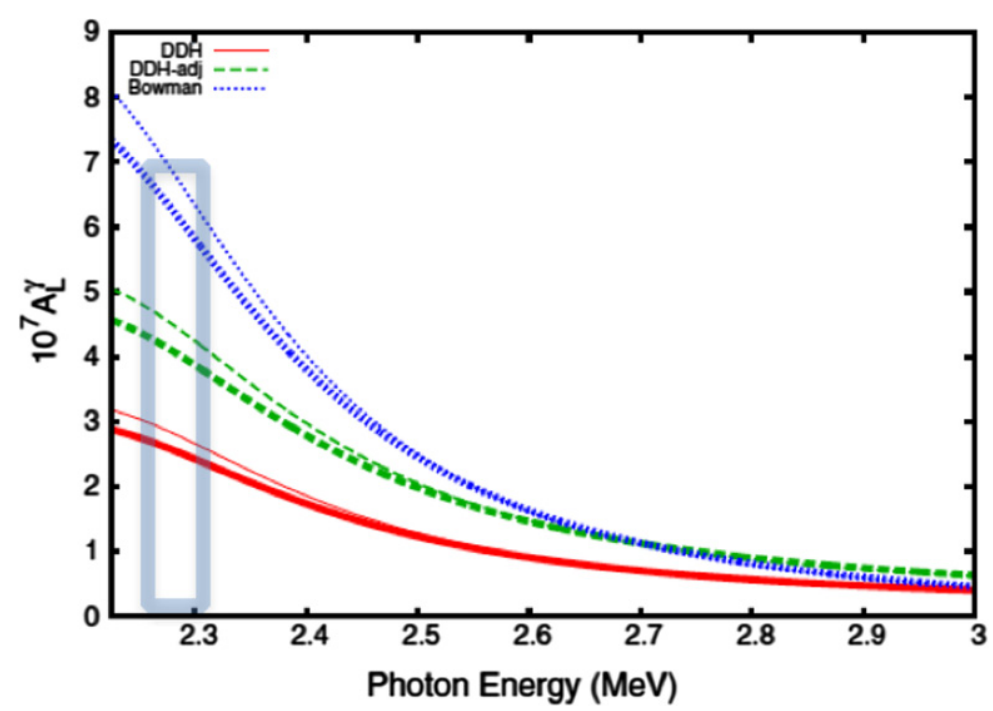

Figure 12. Plot of the calculated $A_{L}^{\gamma}$ for parity violating photodisintegration of the deuteron as a function of photon energy. The lowest photon energy on the plot is the breakup threshold value corresponding to the deuteron binding energy. Reprinted figure with permission from [145], Copyright (2014) by the American Physical Society. The curves represent next-to-leading-order EFT calculations using three different sets of hadronic weak coupling constants, which are labeled DDH, DDH-adj, and Bowman. DDH represents the DDH 'best values' [146]. DDH-adj refers to a set in which two combinations of $\rho$ and $\omega$ couplings are fit to data on the $\vec{p} p$ longitudinal asymmetry, while the remaining couplings take the DDH 'best values' [147]. The set labeled Bowman is obtained by fitting the parity violating couplings to a variety of available data [148]. The optimum energy window for the measurement is indicated by the rectangle.

parameterizations of the hadronic weak coupling constants is plotted as a function of photon energy in figure 12. The FOM quantity that is optimized for obtaining the best statistical accuracy per hour of beam time is FOM $=\left(A_{L}^{\gamma}\right)^{2} \sigma$, where $\sigma$ is the unpolarized photodisintegration cross section. The FOM computed for various parameterizations of the hadronic weak coupling constants are plotted as a function of photon energy in figure 13. This analysis indicates that the experiment should ideally be performed in the photon energy region between 2.26 and $2.30 \mathrm{MeV}$. In this energy window, the average cross section and $A_{L}^{\gamma}$ are $600 \mu \mathrm{b}$ and $4 \times 10^{-7}$, respectively.

A schematic diagram of the concept for the experiment setup is shown in figure 14. The neutrons from the photodisintegration reaction in the liquid deuterium (LD) target are detected in an array of ${ }^{3} \mathrm{He}$ ionization tubes embedded in a cylindrical polyethylene moderator that surrounds the target. The background due to interactions of scattered $\gamma$ rays in the ${ }^{3} \mathrm{He}$ ionization tubes is determined using an array of ${ }^{4} \mathrm{He}$ ionization tubes located outside the inner layers of ${ }^{3} \mathrm{He}$ ionization tubes. About $75 \%$ of the $\gamma$ rays incident on the $21 \mathrm{~cm}$ long LD target are transmitted through the target. The $\gamma$-ray beam is collimated to a diameter of $10 \mathrm{~mm}$, and the inner diameter of the ${ }^{3} \mathrm{He}$ ionization tube array is $6 \mathrm{~cm}$. The ionization tubes are $50 \mathrm{~cm}$ long and centered about the LD target. With this target thickness, detector geometry, and a $\gamma$-ray beam intensity of $10^{10} \gamma / \mathrm{s}$ on target in the above energy window, a statistical accuracy of $\pm 10^{-7} \mathrm{can}$ be obtained with about $9000 \mathrm{~h}$ of beam time. 


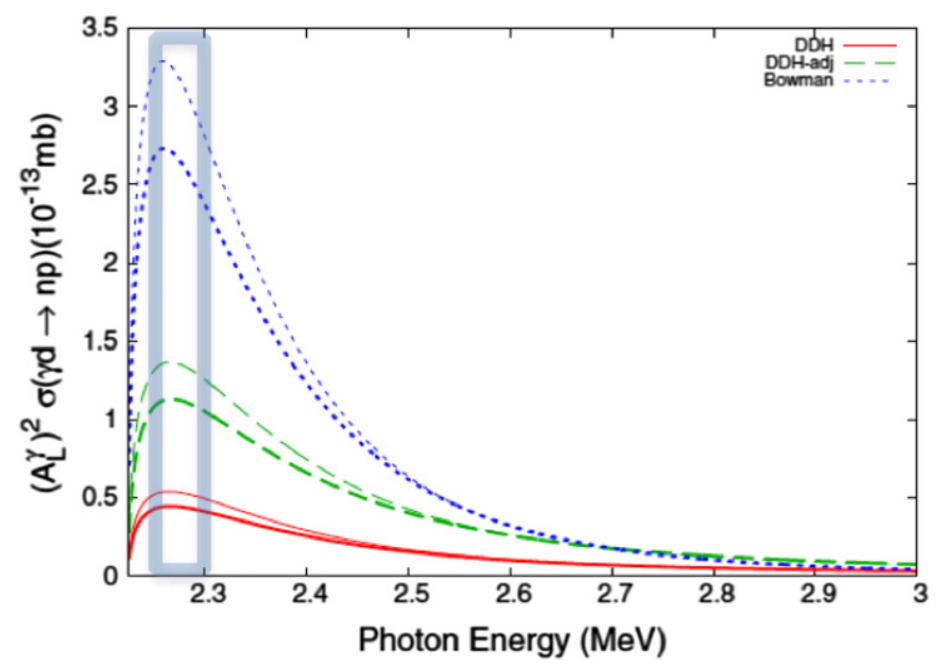

Figure 13. Plot of the calculated FOM for PV photodisintegration of the deuteron as a function of photon energy. The lowest photon energy on the plot is the breakup threshold value corresponding to the deuteron binding energy. Reprinted figure with permission from [145], Copyright (2014) by the American Physical Society. The curves follow the legend given in figure 12. The optimum energy window for the measurement is indicated by the rectangle.

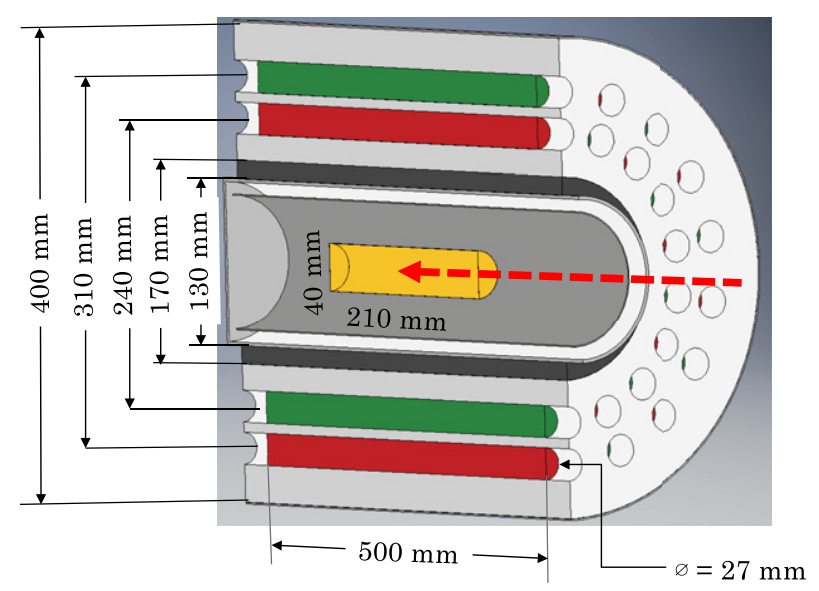

Figure 14. Schematic diagram of the experimental concept for the $A_{L}^{\gamma}$ measurement for PV photodisintegration of the deuteron.

The technical demands to minimize $\gamma$-ray helicity-dependent changes in the phase space of the $\gamma$-ray beam are comparable to those encountered at the polarized electron injector section at JLab in the parity violating electron scattering. Developing the capability for delivering polarized $\gamma$-ray beams to targets with the stability and precision required to perform $10^{-7}$ asymmetry measurements will demand focused and dedicated resources for R \& D work and implementation of the supporting systems. Suggested beam parameters for studying PV in deuteron photodisintegration are shown in table 4. 
Table 4. Suggested beam parameters for the $A_{L}^{\gamma}$ measurement in PV deuteron photodisintegration.

\begin{tabular}{lc}
\hline Parameter & Value \\
\hline Energy & $2.25-2.30 \mathrm{MeV}$ \\
Flux & $10^{10}$ \\
$\Delta E / E$ & 0.001 to $0.01 \mathrm{FWHM}$ \\
Polarization & Circular \\
Diameter & $10 \mathrm{~mm}$ on target \\
Time structure & $10 \mathrm{~Hz}$ polarization flip \\
\hline
\end{tabular}

3.3.2. Hadronic parity violation in light nuclei. The size of the expected parity violating asymmetries from reactions in few-nucleon systems induced with photons at $\mathrm{MeV}$ energies are generally around several times $10^{-7}$. For such experiments, achieving the required systematic accuracy is a formidable challenge, especially at the initial ramp-up stage of an NGLCGS facility. This motivates us to look for parity violating asymmetries that are larger, and therefore easier to measure, even if the interpretation is not as theoretically clean as in few-nucleon systems. Certain light nuclei possess doublets with narrow energy spacings in the 10 to $100 \mathrm{keV}$ range between levels of opposite parity. Parity-odd asymmetries can be amplified by several orders of magnitude by the interference of such parity doublets states involved in photoninduced reactions. There are a half dozen such nuclei (some of which were investigated using charged-particle beams a few decades ago) [117] whose parity-odd asymmetries could be measured using the ultra-high intensity mono-energetic polarized photon beam at the NGLCGS. To develop the technical infrastructure and scientific expertise among the staff, a PV program at such facilities should start with an asymmetry measurement on parity doublets in nuclei, where the parity violating asymmetry is several orders of magnitude larger than that in fewnucleon systems. The experiment could measure either the helicity dependence in the total cross section using transmission or the asymmetry of the fluorescence from the doublet using a HPGe detector array. Examples of candidate nuclei and estimated amplification factors for parity violating asymmetries in nuclear resonance fluorescence are described by Titov et al [149].

Either parity-odd asymmetry can be calculated in a simple way in terms of the energies and widths of the states and the parity-odd matrix elements of interest. PV in states which are particle unstable will be difficult to handle theoretically. However, as the theoretical tools to calculate PV in these nuclei improve, we can identify one or more candidate nuclei for a focused effort involving both theory and experiment.

Although the parity violating asymmetry is enhanced in the parity doublet systems, the photonuclear cross section is small compared to Compton and pair production cross sections in the energy range of interest below the GDR. The beam requirements are therefore still challenging, as shown in table 5 .

\subsection{Nuclear and homeland security}

A next-generation Compton $\gamma$-ray beam facility can contribute to the R \& D of techniques and technologies for applications in homeland security, nuclear safeguards, and medicine. The facility should have the infrastructure that supports measurements that contribute to the nuclear databases important in supporting the development of technologies and techniques in the above areas. Equally important, the facility should have a target area that is equipped for evaluating concepts for $\gamma$-ray beam interrogation of cargo, nuclear fuel, and special assemblies. 
Table 5. Suggested beam parameters for parity violating asymmetry measurements in parity doublets.

\begin{tabular}{lc}
\hline Parameter & Value \\
\hline Energy & $1-20 \mathrm{MeV}$ \\
Flux & $10^{9}$ at $0.1 \%-1 \% \mathrm{FWHM}$ \\
Polarization & Circular \\
Diameter & $10 \mathrm{~mm}$ on target \\
Time structure & $10 \mathrm{~Hz}$ polarization flip \\
\hline
\end{tabular}

Safeguarding the nation against evolving threats that involve the use of special nuclear materials (SNM) requires continued innovations in the procedures and technologies used to inspect cargo at ports of entry into the United States. Research frontier areas include the development of systems for $\gamma$-ray and neutron-beam interrogation of cargo for shielded SNM, new costeffective materials for $\gamma$-ray and neutron detection, and improved techniques and supporting technologies for applications of nuclear forensics in the analysis of interdicted materials. The beam capabilities and technical infrastructure of next generation $\gamma$-ray beam facilities should support the following research: (1) evaluation of $\gamma$-ray beam interrogation techniques and technology concepts, (2) filling gaps in the photonuclear reaction database needed in methods for identification of SNM, including the development and maintenance of online databases, and (3) testing, characterizing, and calibrating $\gamma$-ray and neutron detectors. The infrastructure for photonuclear data measurements should be optimized for nuclear resonance fluorescence (NRF), photo-neutron reactions, and photofission.

Each nucleus with $Z>2$ is characterized by unique excited states. These states have very narrow widths (rarely more than a fraction of an $\mathrm{eV}$ ) and can be populated by absorption of photons of the appropriate energy. When such an excited state decays, characteristic $\gamma$-rays are emitted with unique and well-defined energies. The NRF method of isotope identification, shown in figure 15, is based on these observations. The main advantage of this method over other beam-based techniques is that both the excitation and the de-excitation processes proceed via the electromagnetic interaction, which is well understood. Because of the low angular momentum transferred in photonuclear reactions, photon-induced nuclear excitations are mostly electric dipole ( $E 1)$, magnetic dipole $(M 1)$, and to a lesser extent electric quadruple (E2) transitions from the ground state. If the excited state is unbound to emission of strongly interacting particles (e.g., neutrons, protons, or alpha particles), the photon decay is not strong enough to be observed in competition with particle decay channels. Hence, most of the useful states are positioned below the particle separation energies of around 6 to $8 \mathrm{MeV}$, and they generally have ground state radiative widths $\left(\Gamma_{\gamma}\right)$ of $10-100 \mathrm{meV}$.

There are two main approaches to identifying nuclei using NRF: (1) detection of scattered characteristic $\gamma$-rays, and (2) resonance absorption or the observation of flux removal from the $\gamma$-ray beam by resonance nuclear scattering. In the latter method, resonance absorption creates notches in the energy spectrum of the transmitted beam at energies characteristic of the nuclei encountered by the beam. Bertozzi and Ledoux proposed this method for applications in scanning seagoing cargo containers [150]. Detection of these notches provides the signature for identifying the isotopes through which the beam has passed. The application of the notch technique with nearly mono-energetic $\gamma$-ray beams has been studied recently [151], and a recent measurement performed using the $\gamma$-ray beam at $\mathrm{HI} \gamma \mathrm{S}$ demonstrates that for $\gamma$-ray beams with a small energy spread, such as the 5\% FWHM at $\mathrm{HI} \gamma \mathrm{S}$, the probability of notch refilling due to small-angle scattering is likely to be negligible for most cargo inspection scenarios [152]. 


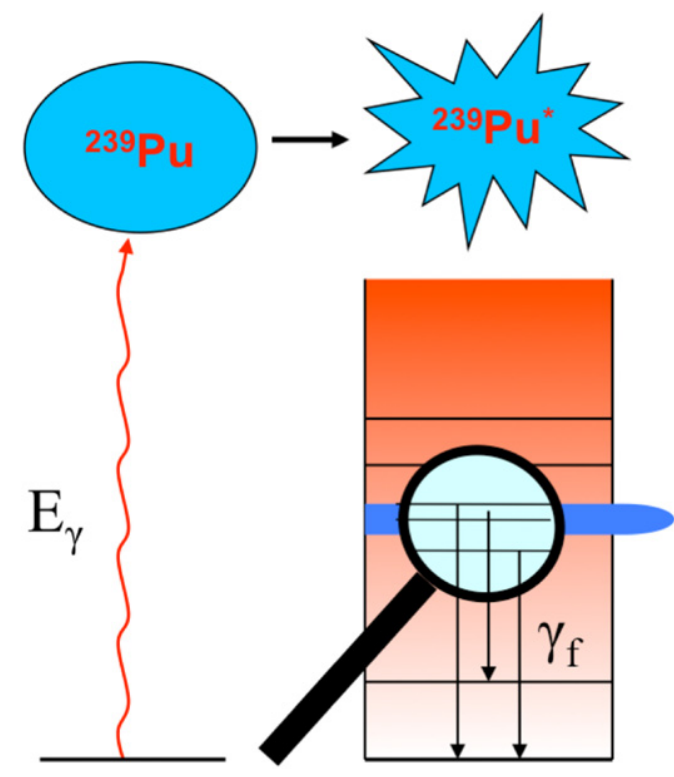

Figure 15. Schematic diagram of the NRF technique. First the incident photon is absorbed by a nucleus at certain resonance energies characterized by the parameters $E_{\mathrm{i}}$, $\mathrm{J}_{\pi}, \Gamma_{\mathrm{i}}$. Then the excited state quickly re-emits $\gamma$-rays at the same or a different energy in a decay pattern that is unique for each type of nucleus. The MeV energy scale is high enough to penetrate heavily shielded containers, thus permitting non-destructive, unambiguous isotope identification and quantitative mass determination.

Beams at a next-generation laser Compton $\gamma$-ray source will enable expansion of the experimental studies of the notch technique reported by Hagmann et al [152]. The goals of such work would include: (1) comparison of signal-to-background ratio of the notch technique to that for detection of scattered $\gamma$-rays for high $Z$ nuclei and a variety of cargo configurations; (2) measurements of effective NRF cross sections for selected isotopes; (3) assessments of performance quantities for the notch technique for simple cargo arrangements, in order to benchmark computer models of the setup; and (4) evaluations of the performance of the notch technique implemented with low-resolution high-efficiency $\gamma$-ray detectors, such as $\mathrm{LaBr}_{3}$ scintillators. The performance quantities include the signal-to-background ratio, the number of signal counts per $10^{9}$ incident $\gamma$-rays, and the radiation levels in the proximity of the cargo.

The driving principle of the notch technique is the analysis of the energy distribution of the beam transmitted through the sample material. The resonance absorption of the $\gamma$-rays by the nuclei in a sample material creates notches in the transmitted beam at the resonance energies. Because the width of the notch (or absorption peak) in the beam-energy profile is typically less than $1 \mathrm{eV}$, direct detection of the notch is not possible with current $\gamma$-ray-detector technology, but the natural line width of $\gamma$-ray transitions in nuclei is used to overcome this technical challenge [150]. The comparison of the beam-flux normalized NRF spectrum on a target measured using the beam transmitted through the cargo sample with that measured with the sample removed gives a measure of the resonance absorption of the cargo material for the nuclei in the downstream target. The $\gamma$-ray beam flux transmitted through the sample at the energy $E_{\mathrm{r}}$ of a resonance in a nucleus in the sample is given by

$$
\phi=\phi_{0} \exp \left(-\sigma_{\text {eff }} \rho x\right)
$$




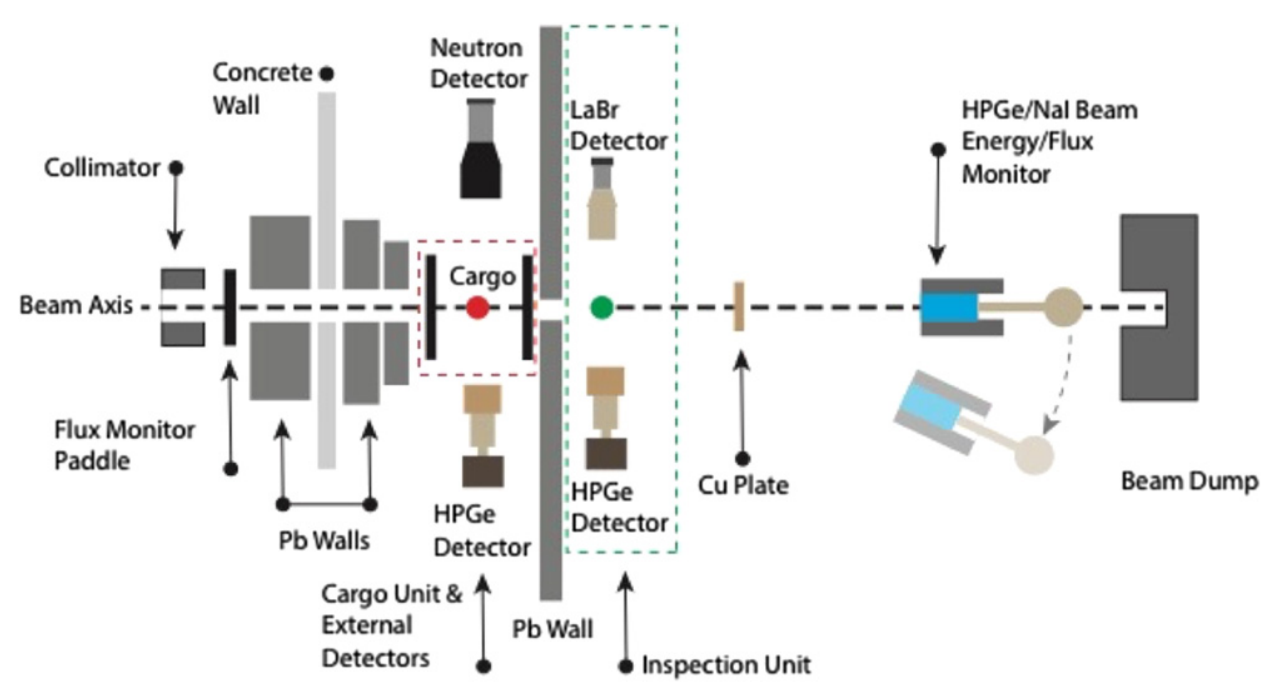

Figure 16. Schematic diagram of the experimental setup for evaluating techniques for remote identification of nuclei using $\gamma$-ray beams.

In this equation $\phi_{0}$ is the $\gamma$-ray beam flux incident on the sample at energy $E_{\mathrm{r}}, \phi$ is the transmitted beam flux at energy $E_{\mathrm{r}}, \sigma_{\text {eff }}$ is the effective nuclear resonance absorption cross section, $\rho$ is the density of the nuclei associated with the resonance in the sample, and $x$ is the thickness of the sample. The quantity $\sigma_{\text {eff }}$ includes effects from Doppler broadening caused by the thermal motion of the nuclei in the sample.

A schematic diagram of the experimental setup for the notch-technique evaluation measurements is shown in figure 16. The time structure of the beam enables the backgrounds uncorrelated with the beam to be measured while collecting beam-induced reaction data and determining the velocity of neutrons emitted from the target via time-of-flight (TOF) measurements. The beam-shaping collimator shields the experimental setup from the radiation produced by the beam using lead and concrete walls indicated in the drawing. The standard collimator diameter in these measurements will be around $25 \mathrm{~mm}$ ( 1 inch). These studies will be performed using circularly polarized $\gamma$-ray beams. At $\gamma$-ray beam energies below $7 \mathrm{MeV}$, the collimator material should be lead, and at higher energies the collimator should be made of aluminum to reduce neutron production via the $(\gamma, n)$ reaction on the collimator. The sample materials being studied are placed between the steel plates in the area labeled cargo in the diagram. The energy spectrum of the beam transmitted through the sample should be analyzed using the NRF measurement setup in the area labeled inspection unit. The cargo arrangement will nominally contain targets that are one to several $\mathrm{cm}$ thick. The analysis foils will normally be a few mm thick. The collimated beam flux before the cargo will be monitored e.g., with a thin plastic scintillator paddle. The absolute beam flux can be measured by Compton scattering from a copper plate into an HPGe detector. The $\gamma$-rays emitted by the cargo sample are detected by HPGe detectors positioned at scattering angles of at least $90^{\circ}$. At $\gamma$-ray beam energies above the neutron separation energy, the fast neutrons emitted from the sample will be detected. The energies of the neutrons are determined by TOF analysis. Both HPGe and $\mathrm{LaBr}_{3}$ detectors are used to detect the $\gamma$-rays emitted by the witness target in the inspection unit. The $\mathrm{LaBr}_{3}$ detectors offer a possible alternative to HPGe detectors in field application. 


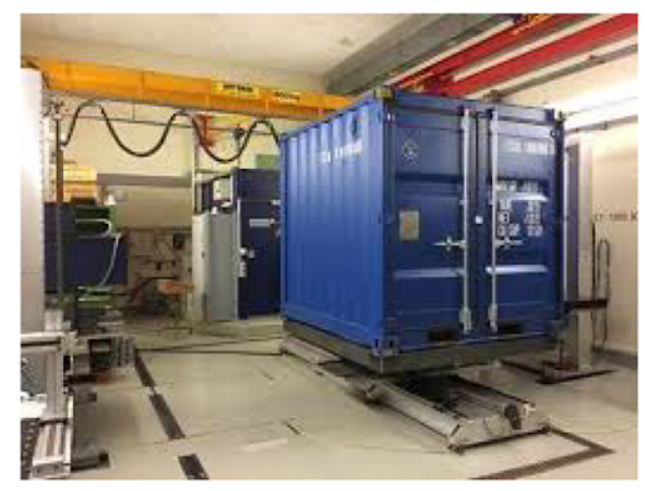

Figure 17. Movable support structure for concept evaluation. Its movement degrees of freedom allow 3D $\gamma$-ray beam scanning for 'real-world' scenarios. Adapted from [153]. CC BY-SA 4.0.

Two distinct types of measurements can be performed using this system: (1) determination of $\sigma_{\text {eff }}$ for several states in key nuclei such as ${ }^{238} \mathrm{U},{ }^{239} \mathrm{Pu},{ }^{235} \mathrm{U},{ }^{208} \mathrm{~Pb},{ }^{12} \mathrm{C},{ }^{14} \mathrm{~N},{ }^{16} \mathrm{O}$, and ${ }^{24} \mathrm{Mg}$; and (2) evaluation of techniques for remote isotope analysis of cargo. The same nuclei can be studied in both measurements. In addition to determining signal-to-background ratios for the notch measurements (inspection station) and detection of the scattered $\gamma$-rays (cargo setup), the radiation levels inside and just outside the target area should be recorded during measurements, especially when evaluating beam-based interrogation concepts. In addition, the facility should have the capability for measuring the energy spectra of the fast neutrons and $\gamma$-rays at several locations in the target area during data collection under various conditions.

The target area for nuclear and homeland security applications should be equipped with a support system for imaging and scanning cargo-size containers, e.g., as the system shown in figure 17, which is used in the European project for developing a system for automated comparison of X-ray images for cargo scanning [153]. This system should allow 'real-world' setups to be moved along multiple axes and rotated to allow beam scans. The target room should be equipped with detectors and instrumentation with the capabilities for large-area measurements of

- NRF

- Photofission: prompt and delayed neutrons and $\gamma$-rays

- Imaging: transmission, backscattering, and NRF with linearly polarized $\gamma$-ray beams.

\section{Nucleon structure and low-energy QCD}

The nucleon sits roughly halfway on the ladder of distance scales of relevance for contemporary nuclear physics. Going up the ladder, ab initio calculations that use protons and neutrons and the forces between them as building blocks predict the properties of nuclei as large as ${ }^{100} \mathrm{Sn}$. Going down the ladder, lattice simulations that employ the fundamental QCD degrees of freedom—quarks and gluons — are now performed at the physical quark mass, reproduce a variety of hadron properties, and provide an increasingly detailed picture of its internal structure.

However, much of that internal structure is not resolved inside a typical nucleus under terrestrial conditions. The calculations of nuclei and nuclear matter described in the first paragraph use emergent QCD degrees of freedom: protons, neutrons, pions, and the delta resonance. The 
interactions of these particles are not always well constrained by, e.g., NN scattering data. Understanding the properties, structure, and interplay of these low-energy QCD degrees of freedom is crucial to continued progress in our field.

The $\gamma$-ray beam of a next-generation laser Compton source is ideal for the examination of nucleon structure in this 'low-energy QCD' domain. Its electromagnetic field couples to the distribution of charge and magnetization inside the nucleon. At the lower end of the NGLCGS energy range, $\omega \lesssim 50 \mathrm{MeV}$, the photons will not resolve the nucleon's internal structure. But as $\omega$ is increased beyond that, different degrees of freedom become important in determining the photon response of neutrons and protons. There are two regions of particular note: (1) around $\omega \approx 150 \mathrm{MeV}$, the pion-production channel opens; and (2) once $\omega \approx 300 \mathrm{MeV}$ the photon field is resonant with the lowest excited state of the nucleon, the delta(1232) resonance.

In this chapter, we discuss two different NGLCGS experimental programs that probe this physics. In Compton scattering, the incoming photons of the $\gamma$-ray beam induce oscillations in the nucleon's internal charge and magnetization distributions. These, in turn, cause the reemission of radiation, with a strength that is proportional to the frequency-dependent coupling between the photon and the internal oscillation. Compton scattering is thus unique among probes of nucleon structure: it can activate pions and other effective degrees of freedom inside the nucleon even at energies where these cannot be liberated as physical particles in the final state.

Indeed, nucleon Compton scattering involves a fascinating interplay of different QCD mechanisms. The pions' special status as (pseudo-)Goldstone bosons of QCD means that the nucleon polarizabilities which parameterize the low-frequency nucleon response to the photon should bear the imprint of this Goldstone-boson physics. Those pion effects are complemented by those of the delta excitation-especially for magnetic properties. Again, since photons activate virtual excitations, the delta affects the Compton response even at energies well below the resonance. At higher energies, nucleon Compton scattering is sensitive to the still-mysterious scalar sector of QCD and to higher-energy nucleon resonances.

The impact of virtual degrees of freedom on Compton scattering makes it an excellent place to learn how different hadronic degrees of freedom become active as the photon beam energy is increased. NGLCGS will be uniquely positioned to be a scale-scanning facility, examining the entire photon-energy range of relevance for low-energy QCD. Beyond these energies, where the wavelength decreases further, there is a transition to the physics examined by high-energy facilities, including a future EIC, where quarks and gluons become a more efficient way to think about electromagnetic response. But the fundamental QCD degrees of freedom are not efficient to model nuclei, whose physics is dominated by hadron dynamics at distance scales of 1-2 fm, see figure 18 .

Once $\omega \gtrsim 150 \mathrm{MeV}$, pions can be produced as physical particles in the final state. We therefore plan that Compton-scattering studies at NGLCGS be complemented by an aggressive program to measure this reaction. While much data in this regime already exists from LEGS, Mainz, and other facilities, important questions remain. In particular, the flux and monochromaticity of the NGLCGS beam will make possible pion-production experiments that can test the limits of isospin symmetry in pion-nucleon and NN interactions. A precise understanding of isospin violation is becoming a key input for models of nuclei, as facilities like FRIB and RIKEN push ever further into the neutron-rich frontier.

\subsection{Compton scattering}

Compton scattering from hadrons provides unique access to the symmetries and dynamics of the charges and currents constituting the low-energy degrees of freedom inside the nucleon 


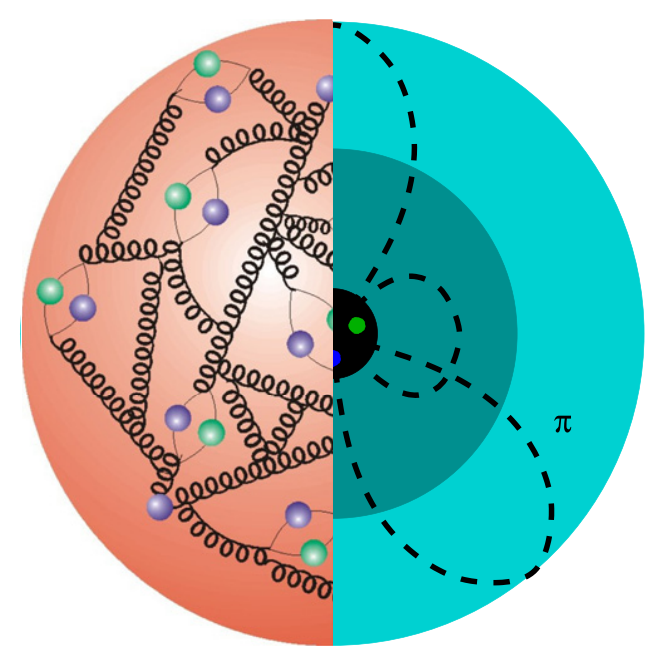

Figure 18. The appropriate degrees of freedom with which to describe nucleon structure depend on the distance scale at which it is probed: quarks and gluons at high energies (left); or nucleons, their pion clouds and nucleonic excitations at low energies (right).

$[16,154-158]$. This information can be encoded in the proportionality factors between the incident field and the induced multipole: the dynamical, energy-dependent polarizabilities of the nucleon. Intuitively speaking, they measure the response of the low-energy degrees of freedom in the nucleon in transitions $X l \rightarrow Y l^{\prime}$ of definite multipolarity for a real photon with non-zero frequency $\omega$, and $l^{\prime}=l \pm\{0 ; 1\}$. After subtracting the effects of a point-like nucleon, one expands the amplitude as $\left(X, Y=E, M ; T_{i j}=\frac{1}{2}\left(\partial_{i} T_{j}+\partial_{j} T_{i}\right)\right.$; and $\left.T=E, B\right)$ :

$$
\begin{array}{r}
2 \pi\left[\alpha_{E 1}(\omega) \vec{E}^{2}+\beta_{M 1}(\omega) \vec{B}^{2}+\gamma_{E 1 E 1}(\omega) \vec{\sigma} \cdot(\vec{E} \times \dot{\vec{E}})+\gamma_{M 1 M 1}(\omega) \vec{\sigma} \cdot(\vec{B} \times \dot{\vec{B}})\right. \\
\left.\quad-2 \gamma_{M 1 E 2}(\omega) \sigma^{i} B^{j} E_{i j}+2 \gamma_{E 1 M 2}(\omega) \sigma^{i} E^{j} B_{i j}+\ldots \text { (higher multipoles) }\right] .
\end{array}
$$

For $\omega$ up to about $300 \mathrm{MeV}$, just six dynamical polarizabilities characterize the nucleon response: two scalar polarizabilities, $\alpha_{E 1}(\omega)$ and $\beta_{M 1}(\omega)$, for electric and magnetic dipole transitions, and four spin polarizabilities $\gamma_{\mathrm{i}}(\omega)$ which are addressed in more detail below. The zero frequency values, $\alpha_{E 1}(\omega=0)$ etc, are often called the 'polarizabilities'.

As explained above, Compton scattering has a special role as a scale-scanning probe of the nucleon's internal degrees of freedom. In the NGLCGS energy range, the six dynamical polarizability functions above serve as benchmarks of our understanding of the way in which short-distance QCD dynamics and the consequences of chiral symmetry breaking-especially as encoded in pionic excitations - combine to produce nucleon structure. Subtle alterations in this interplay then produce differences between proton and neutron values, and precise polarizability numbers (including nucleon differences) are crucial ingredients in attempts to understand the neutron-proton mass difference and the extraction of nuclear radii from the Lamb shift in muonic atoms that produced the 'proton-radius puzzle' [158-162].

Our goal is to map out the interplay of low-energy QCD mechanisms that is encoded in the dynamical polarizabilities of the proton and neutron. To this end, we advocate a series of NGLCGS Compton-scattering experiments with both polarized and unpolarized beams and targets. The targets are the proton, deuteron, ${ }^{3} \mathrm{He}$ and ${ }^{4} \mathrm{He}$, see figure 19 . This means 


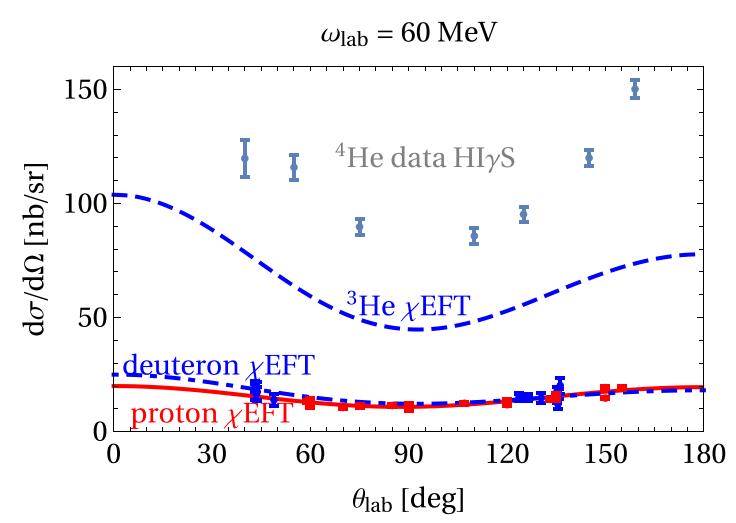

Figure 19. Predicted (curves) and measured (data points) elastic Compton cross sections at $\omega_{\text {lab }}=60 \mathrm{MeV}$ for the different targets discussed in this chapter.

NGLCGS will do challenging experiments on targets where nuclear effects are absent or clearly under control, while also examining nuclei with larger Compton cross sections and relying on theory for a reliable extraction of nucleon-level information. This interplay of experimental signal strength and calculational complexity across different targets will test the unified picture of Compton scattering from light nuclei that has been built up over the last twenty years.

As discussed in the 2015 NSAC/DOE and 2017 NuPECC long-range plans [163-165], there has been significant progress in our understanding of nucleon polarizabilities over the last decade; see also section 4.1 .3 below. This has occurred because of a synergistic community effort that employed diverse nuclear-physics tools so that Compton data on protons and light nuclei now confronts the emerging LQCD computations described in section 4.1.1, with chiral effective-field theory ( $\chi \mathrm{EFT})$ and dispersion-relation calculations acting as a bridge between the lattice and the laboratory (see section 4.1.2).

In the next decade, attention will turn to the spin polarizabilities, which so far are largely unexplored. These parameterize the optical activity of the nucleon-the stiffness of the nucleon's spin degrees of freedom (figure 20). They therefore complement experiments at higher-energy facilities that illuminate how the nucleon spin is built up from quark and gluon spin and orbital angular momentum. In an intuitive picture, $\gamma_{E 1 E 1}(\omega)$ and $\gamma_{M 1 M 1}(\omega)$ encode how the electromagnetic field produced by the nucleon spin leads to bi-refringence, as in the classical Faraday effect. Until the recent pioneering experiment of references [166, 167], only the linear combinations $\gamma_{0}$ and $\gamma_{\pi}$ of spin polarizabilities that enter scattering at $0^{\circ}$ and $180^{\circ}$ had been determined [156, 157]. For the proton, even these had substantial uncertainties, with conflicting results from MAMI and LEGS. For the neutron, even less is known. We will discuss the current status of all scalar and spin polarizabilities in section 4.1.3.

4.1.1. Insights from lattice $Q C D$. First though, we address how such measurements are related to QCD. Polarizabilities can be computed directly on the lattice. But since they are secondorder in electromagnetic fields and furthermore diverge near the chiral limit, they provide a considerable challenge, and thereby a stringent test of lattice methods. Similar techniques can then be trusted for QCD input to double-beta decay, dark-matter detection, and beyond-thestandard-model currents in nucleons and nuclei. 
S S S S S S S S S

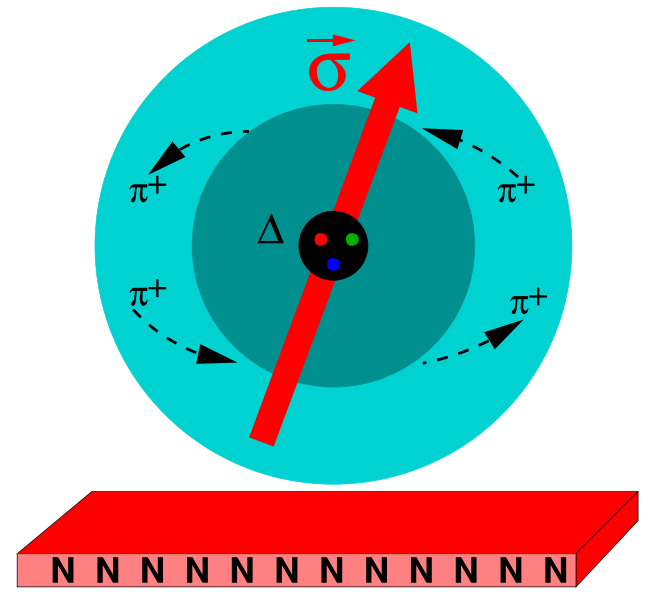

Figure 20. A cartoon that illustrates how the response of the nucleon, including its spin, to an applied magnetic field provides information on the nucleon's composition in terms of low-energy degrees of freedom.
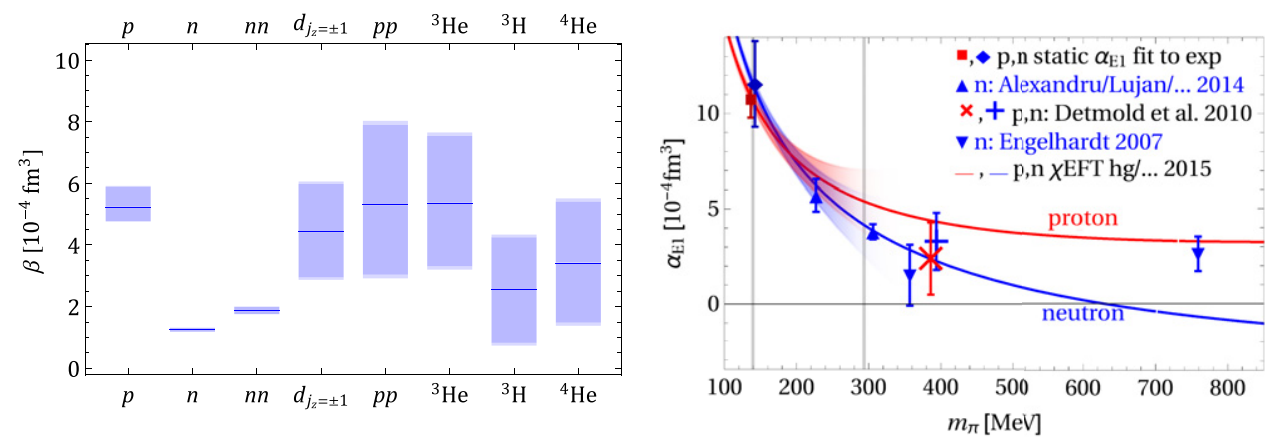

Figure 21. Left: magnetic polarizabilities of nucleons and light nuclei extracted from LQCD computations performed at $m_{\pi}=800 \mathrm{MeV}$ [174]. Right: pion-mass dependence of the electric polarizabilities in $\chi \mathrm{EFT}$, with experimental extractions at the physical point and available lattice results [178]

The recent success of the background-field technique used for these calculations has led to a strong effort to refine computations of nucleons and nuclei in uniform electromagnetic fields [168-176]. Results from the NPLQCD collaboration shown in figure 21 even include the first computations of magnetic properties of light nuclei. There is also a pioneering computation of $\gamma_{E 1 E 1}$ by Engelhardt [177]. Both show that lattice and experimental uncertainties on these quantities are commensurate, and have the opportunity to be reduced in parallel.

Currently, the most significant systematic error is that computations are performed at unphysically large pion masses, altering the dynamical response to applied electromagnetic fields. However, recent cross-fertilization between LQCD and phenomenology furthers the 
goal of exposing the role of chiral symmetry in low-energy QCD [178]. For magnetic polarizabilities, the pion mass provides a dial that alters the relative weight of diamagnetic and paramagnetic contributions, leading to a deeper understanding of the underlying effective degrees of freedom. Analysis of LQCD results with $\chi$ EFT, moreover, provides a consistency check that is independent of using Compton data. In turn, high-accuracy experiments in single and few-nucleon systems provide crucial constraints on LQCD computations.

4.1.2. Extracting polarizabilities from data. Equation (4.1) shows that, near $\omega=0$, the effects of the scalar polarizabilities in cross sections are $\propto \omega^{2}$, and $\propto \omega^{3}$ for spin polarizabilities. With increasing energy, polarizability contributions become more prominent. Resonances and particle-production thresholds lead to further enhancements. When inelastic channels are open, the dynamical polarizabilities become complex, and their imaginary parts are directly related to pion photoproduction. That allows, for example, an indirect exploration of some currently ill-determined $n \pi$ photoproduction multipoles [179].

Since data are taken at (ideally several) nonzero energies, the '(static) polarizabilities' $\alpha_{E 1}(\omega=0)$ etc follow from an extrapolation of results at nonzero energies and compress the richness of such information into just a few numbers. A sophisticated and reliable understanding of the energy dependence of these functions is required, so that the static values can be extracted from data taken at energies from 70 to $250 \mathrm{MeV}$. A good understanding of the energy dependence induced by pion-production and the $\Delta(1232)$ resonance is therefore mandatory.

Recently, an open letter of theorists with backgrounds in several variants of dispersion relations and effective field theories summarized the theory status as follows [180]:

(a) Static scalar polarizabilities can be extracted from future data well below the pionproduction threshold with high theoretical accuracy.

(b) Data around and above the pion production threshold show increased sensitivity to the spin polarizabilities and will help to understand and resolve some discrepancies between different approaches.

(c) All theoretical approaches resort to well-motivated but not fully controlled approximations around and above the $\Delta(1232)$ resonance. Concurrently, sensitivity to the static polarizabilities decreases substantially. Instead, one studies details of the $\Delta(1232)$ resonance, as well as the degrees of freedom exchanged between photons and the nucleon in the t-channel.

The transition from one regime to another is, of course, gradual rather than sudden.

Neutron polarizabilities are less well determined than proton ones, see equation (4.2). Since both are equal at leading order in $\chi \mathrm{EFT}$, their precise measurement could reveal subtle differences in the pion dynamics around the neutron and proton.

Such effects have fundamental implications: reference [159] pointed out that the subtraction function in their dispersive treatment of the neutron-proton mass difference can be integrated to yield $\alpha_{E 1}^{(\mathrm{p})}-\alpha_{E 1}^{(\mathrm{n})}$. Accurate measurements of the differences of the electric polarizabilities will therefore illuminate the interplay of quark-mass and electromagnetic contributions to $M_{\mathrm{n}}-M_{\mathrm{p}}$.

The neutron dynamical polarizabilities can be determined from Compton scattering on light nuclei. For photon energies below $100 \mathrm{MeV}$, elastic scattering is favored. While the Compton response of the neutron itself is quite small there, it interferes constructively with large pieces of the nuclear amplitude, including the proton Thomson term and the sizable pionexchange currents of bound systems. The importance of the latter for Compton scattering in light nuclei means that measurements of the elastic reaction also provide indirect, non-trivial 
benchmarks for the theoretical description of mesonic contributions to nuclear binding. The different systematics in theory calculations of elastic and quasi-free (QF) Compton scattering on light nuclei make experiments in neutron QF kinematics an attractive complement to measuring the coherent process.

In each case, $\chi$ EFT provides the necessary, model-independent parameterizations of Compton scattering on the various targets described here, including consistency between singlenucleon and pion-exchange currents [16]. And crucially, it does so in a framework where the residual theoretical uncertainties can be reliably estimated. A $\chi$ EFT that is well-matched to the experiments outlined below exists for the proton up to about $250 \mathrm{MeV}$; for the deuteron, up to $120 \mathrm{MeV}$; and for ${ }^{3} \mathrm{He}$, at 50 to $120 \mathrm{MeV}$. Accuracies are $2 \%$ or better up to $\omega \sim m_{\pi}$, and $20 \%$ or better around the $\Delta$ resonance. For each of these nuclei, comprehensive studies of the sensitivities of Compton observables with polarised beams and/or targets, and even for some recoil observables, are available in these regions [181-185]. With present efforts and new experiments on the horizon, a consistent $\chi$ EFT description up to $250 \mathrm{MeV}$ for all light nuclei, including ${ }^{4} \mathrm{He}$, is being pursued vigorously. Results can be expected within the next few years.

4.1.3. Review of recent accomplishments. The 2015 US long range plan states: 'Great progress has been made in determining the electric and magnetic polarizabilities.' The 2018 Particle Data Group numbers for the proton and neutron scalar polarizabilities (in the canonical units of $10^{-4} \mathrm{fm}^{3}$ ) are:

$$
\begin{array}{ll}
\alpha_{E 1}^{(\mathrm{p})}=11.2 \pm 0.4 & \beta_{M 1}^{(\mathrm{p})}=2.5 \pm 0.4 ; \\
\alpha_{E 1}^{(\mathrm{n})}=11.8 \pm 1.1 & \beta_{M 1}^{(\mathrm{n})}=3.7 \pm 1.2 ;
\end{array}
$$

see figure 22. The sums, $\alpha_{E 1}^{(\mathrm{p})}+\beta_{M 1}^{(\mathrm{p})}=13.8 \pm 0.4$ and $\alpha_{E 1}^{(\mathrm{n})}+\beta_{M 1}^{(\mathrm{n})}=15.2 \pm 0.4$ are quite accurately known from the Baldin sum rule and therefore used as constraints in scalar-polarizability determinations [187-189]. This implies that a portion of the error bars are anticorrelated. Particularly notable in the proton case is that efforts by several theory groups, in different frameworks, have led to compatible results, in each of which theory and experimental uncertainties are of similar size [190-192]. For example, the extraction of reference [190] quotes a theory error of \pm 0.3 while the uncertainty stemming from the $\gamma$-proton data itself is \pm 0.35 . In spite of this positive development, there is a disconcerting residual dependence of extractions on the $\gamma$-proton data used [16, 190-193]. This has led to an ongoing discussion about the appropriate criteria for a statistically consistent database. Ultimately, this matter can only be definitively decided by additional high-quality $\gamma$-proton data. If NGLCGS were to start running tomorrow, this would be a pressing problem for it to address. But approved experiments at $\mathrm{HI} \gamma \mathrm{S}$ and MAMI will greatly expand the proton Compton database within the next few years; we expect they will resolve it.

As for the neutron results, a US-led collaboration at MAX-IV added 22 points in 2015, effectively doubling the deuteron's world dataset and reducing the statistical error for the neutron values by $30 \%[194,195]$. Since $\mathrm{HI} \gamma \mathrm{S}$ is now adding precision data, we anticipate that proton and neutron errors will soon be small enough to enable quantitative exploration of the extent to which the proton and neutron deformations in electromagnetic fields differ.

Another US-led collaboration conducted the pioneering measurements of doubly polarized Compton-scattering observables at MAMI and provided the first extraction of proton spin polarizabilities [166, 167, 196-198]. They compare favorably with predictions from two different implementations of $\chi \mathrm{EFT}$ and from dilution refrigerator (DR); see figure 23. In units of $10^{-4} \mathrm{fm}^{4}$ : 


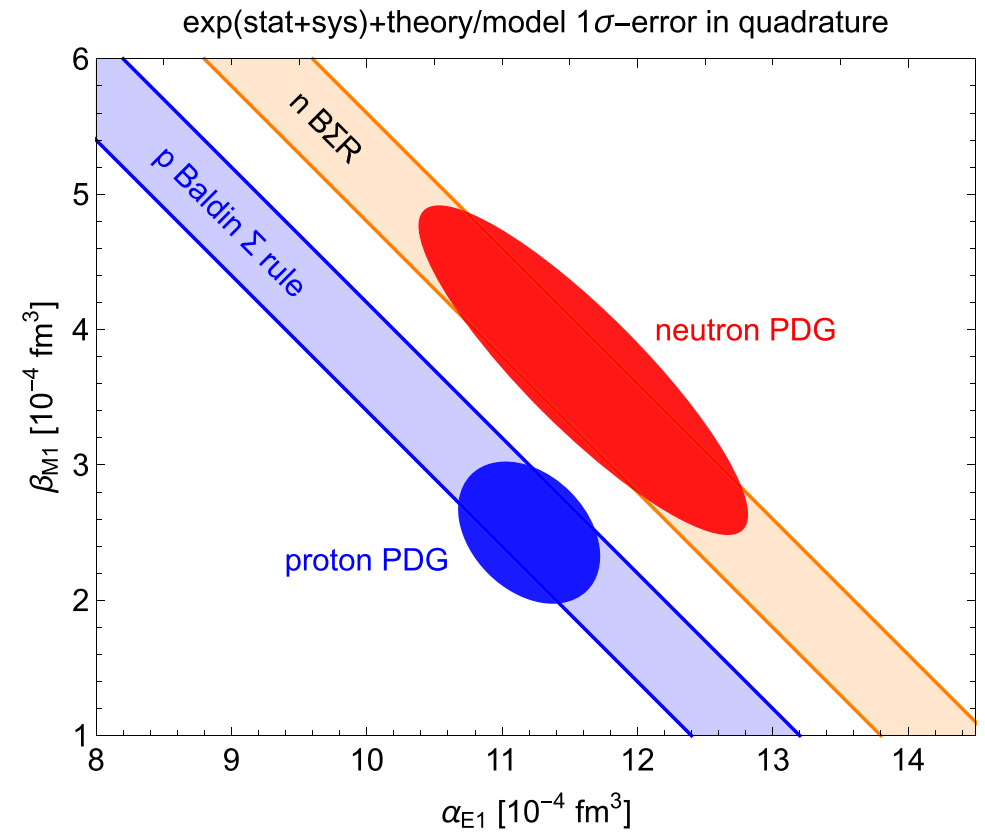

Figure 22. Static scalar polarizabilities (blue: proton; red: neutron); most recent PDG listings. Ellipses represent $1 \sigma$ errors, with statistical, systematic and theoretical errors added in quadrature.

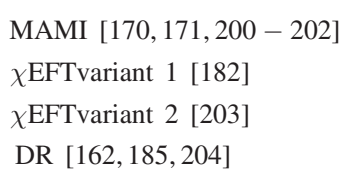

$$
\begin{gathered}
\gamma_{M 1 M 1} \\
3.2 \pm 0.9 \\
2.2 \pm 0.5_{\text {stat }} \pm 0.6_{\text {th }} \\
2.9 \pm 1.5_{\text {th }} \\
{[2.7 \ldots 3.0]}
\end{gathered}
$$

$$
\begin{gathered}
\gamma_{E 1 M 2} \\
-0.7 \pm 1.2 \\
-0.4 \pm 0.6_{\mathrm{th}} \\
+0.2 \pm 0.2_{\mathrm{th}} \\
{[-0.1 \ldots 0.3]}
\end{gathered}
$$

But more work is clearly needed to reduce the experimental uncertainties of $20 \%-170 \%$ and obtain more accurate information on the low-energy response of the nucleon's spin degrees of freedom to electromagnetic fields.

4.1.4. Overall goals of a Compton program. The existing unpolarized proton database, while large in size, is noisy, and the data between $190 \mathrm{MeV}$ and $250 \mathrm{MeV}$ are not of particularly high quality [16]. The deuteron data were markedly improved by Myers et al [194, 195], but the uncertainties are not on a par with the proton ones. Spin polarizabilities are best extracted from data with both polarized beams and polarized targets. For the proton, pioneering doublepolarization data are now available [166, 167, 196-198]; but there is no corresponding data set for a light-nuclear target from which neutron values can be inferred. Indeed, no data, polarized or unpolarized, have been published for ${ }^{3} \mathrm{He}$, which seems to be a promising target in this regard.

Further progress in our understanding of the nucleon's electromagnetic response will thus come on three fronts: 

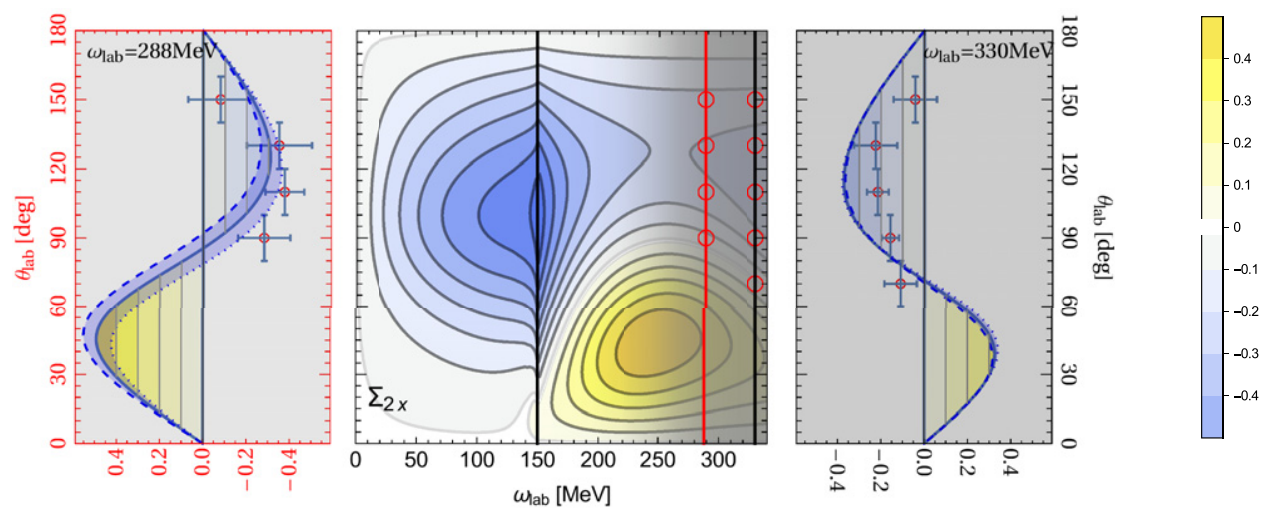

Figure 23. Prediction of the proton double-polarization observable $\Sigma_{2 x}$ with circularly polarized beam and transverse polarized target in $\chi$ EFT [182] compared to the data from MAMI [166, 167, 196-198].
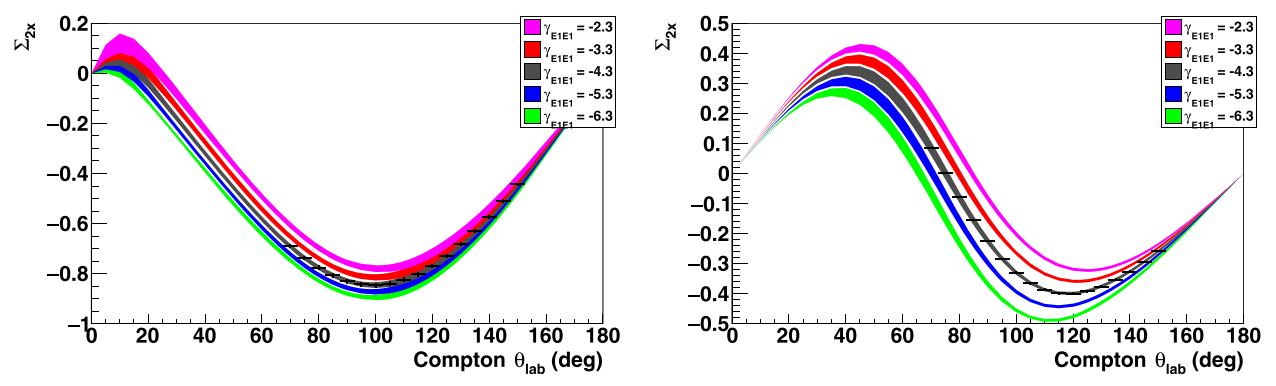

Figure 24. Projected error bars for Compton scattering asymmetries with a transversely polarized proton target at $150 \mathrm{MeV}$ (left) and $290 \mathrm{MeV}$ (right). The colored bands show the predicted asymmetries [183] with $\gamma_{M 1 M 1}, \gamma_{E 1 M 2}$, and $\gamma_{M 1 E 2}$ held fixed, and $\gamma_{E 1 E 1}$ varied as shown in the figure legend.

- Very accurate extractions of neutron scalar polarisabilities that will illuminate differences in neutron and proton internal structure;

- Measurements of the largely unexplored neutron spin polarizabilities;

- Marked improvement of the proton spin-polarizability values, enabling issues requiring precision knowledge of low-energy spin structure to be addressed.

We therefore propose a suite of high-accuracy experiments on protons and light nuclei that, together, will determine the static polarizabilities and map out the dynamical polarizabilities of the neutron and proton over a broad energy range. The result will be an understanding of the role that different QCD effects play in nucleon structure. The experiments are described in section 4.1.5, where each has its own subsection, with individual specifications of beam parameters. We close with sections on the target (section 4.1.6) and beam (section 4.1.7) technology needed to make these experiments a reality.

Several interrelated experiments are necessary since it is unlikely that a single experiment will determine a particular static polarizability with the requisite precision. The program is designed to provide a coherent data set which will dramatically improve extractions and enable unprecedented checks of both experimental systematics and theoretical bias. For all targets, 
high-quality data with carefully formulated correlated and point-to-point systematic errors are needed.

The scalar polarizabilities are best extracted from both unpolarized and polarized experiments where the effect of spin polarizabilities is small, namely at photon energies below $100 \mathrm{MeV}$. Proton targets provide a direct avenue for proton polarizabilities. The deuteron and ${ }^{4} \mathrm{He}$, as isoscalar targets, are sensitive to the average of proton and neutron polarizabilities, $\alpha_{E 1}^{(\mathrm{p})}+\alpha_{E 1}^{(\mathrm{n})}$ etc. In ${ }^{3} \mathrm{He}$, the sensitivity is to $2 \alpha_{E 1}^{(\mathrm{p})}+\alpha_{E 1}^{(\mathrm{n})}$ etc. Experiments on different light nuclei provide important checks of theory systematics, and in particular of the unified $\chi E F T$ description of meson-exchange currents in these nuclei. These binding effects must be subtracted in a model-independent theory approach.

The spin polarizabilities can be extracted at energies around or above $120 \mathrm{MeV}$ by combining precise data and theory with a carefully selected set of double-polarized observables whose sensitivities are dominated by only one or two proton and neutron spin polarizabilities measured over a range of energies; see e.g. references [182, 183]. Again, proton polarizabilities are directly accessible with proton targets, but the isoscalar values from deuteron targets provide valuable cross checks. Since polarized ${ }^{3} \mathrm{He}$ is an effective polarized-neutron target, neutron values can also be determined without knowing their proton counterparts [185].

Inelastic Compton scattering on the quasi-elastic ridge is dominated by the impulse approximation. Measurements of the neutron-photon interaction in QF kinematics therefore provide another avenue to extract neutron polarizabilities. High-quality theory is needed to control the corrections to the QF approximation (for first steps see reference [186]).

Lastly, we point out that a series of measurements over a wide kinematic range is not only necessary for high-accuracy determinations of scalar and spin polarizabilities. It also allows reconstruction of the functions $\alpha_{E 1}(\omega), \beta_{M 1}(\omega)$ and $\gamma_{\mathrm{i}}(\omega)$, yielding the energy dependence of the dynamical polarizabilities, see recent progress in references [191, 192]. This provides stringent tests of our understanding of different low-energy QCD mechanisms and of the way they are manifest in Compton scattering.

\subsubsection{Experiments.}

4.1.5.1. Proton spin polarizabilities. A monochromatic, high flux photon source with $10^{9} \mathrm{\gamma} / \mathrm{s}$, with linear and circular polarizations of approximately $100 \%$, can provide the definitive measurement of the proton spin polarizabilities. Figure 24 shows representative asymmetry data points for the $\Sigma_{2 x}$ asymmetry using a transversely polarized target and circularly polarized photons with $200 \mathrm{~h}$ of running at both $150 \mathrm{MeV}$ and $290 \mathrm{MeV}$. This assumes a frozen-spin target similar to the Mainz design and a $4 \pi \gamma$-ray detector similar to the Mainz crystal ball. The beam conditions are shown in table 6. Figure 25 shows representative asymmetry data points for the $\Sigma_{3}$ asymmetry assuming an unpolarized target and linearly polarized photons. A conservative estimate is that statistical uncertainties in the polarizabilities can be reduced by a factor of ten or more relative to those in the Mainz results.

Comprehensive discussions of theory predictions for the dependence of all double-polarized observables on the spin polarizabilities are provided in references [181-183]. To test the theory dependence of the analysis it will also be important to take data at lower energies, around $150 \mathrm{MeV}$. At incident energies below approximately $250 \mathrm{MeV}$, the recoil proton is undetectable because it does not escape the frozen-spin target cryostat. Without that, it is problematic to separate the Compton signal from backgrounds caused by coherent and QF Compton scattering on nuclear species in the polarized target and from the target cell wall. One way around this problem is to utilize a polarizable polystyrene scintillator as the target, where the scintillation light produced in the target is used to tag a Compton scattering event on the proton. A polarizable active target has been developed at Mainz and shown to function well. 
Table 6. Suggested beam parameters for proton spin polarizability.

\begin{tabular}{lc}
\hline Parameter & Value \\
\hline Energy range & 150 to $300 \mathrm{MeV}$ \\
Flux on target & $10^{9} \mathrm{\gamma} / \mathrm{s}$ \\
Relative energy resolution & $2 \% \mathrm{FWHM}$ \\
Polarization & Linear and circular, $>95 \%$ \\
Beam diameter & $5 \mathrm{~mm}$ on target \\
Time structure & Highest frequency available, pulse width $<1 \mathrm{~ns}$ \\
\hline
\end{tabular}
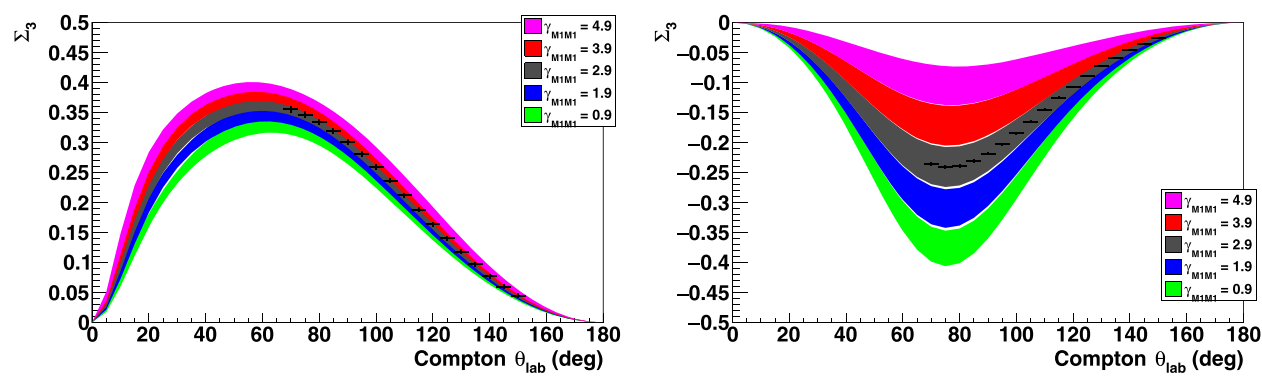

Figure 25. Projected error bars for Compton scattering asymmetries with an unpolarized proton target and linearly polarized photons at $150 \mathrm{MeV}$ (left) and $290 \mathrm{MeV}$ (right). The colored bands show the predicted asymmetries [183] with $\gamma_{E 1 E 1}, \gamma_{E 1 M 2}$, and $\gamma_{M 1 E 2}$ held fixed, and $\gamma_{M 1 M 1}$ varied as shown in the figure legend.

4.1.5.2. Neutron scalar polarizabilities from elastic Compton scattering on deuterium. $\mathrm{HI} \gamma \mathrm{S}$ has recently acquired Compton-scattering data on deuterium at 65 and $85 \mathrm{MeV}$. Extending the current $\mathrm{HI} \gamma \mathrm{S} \gamma d$ data set to energies above $100 \mathrm{MeV}$ and using a significantly higher beam intensity will greatly improve the extracted polarizabilities, since the sensitivity increases at higher energies.

A preliminary plot of the angular distribution of the cross section for ${ }^{2} \mathrm{H}(\gamma, \gamma)^{2} \mathrm{H}$ is shown in figure 26. The inelastic contribution that is observed at backward angles, unresolved in the $\mathrm{HI} \gamma \mathrm{S}$ data, is the largest systematic uncertainty in the present experiment. Part of this resolution limitation is due to the NaI detectors themselves, but part stems from poor incident beam resolution: resolution was sacrificed in order to gain flux. The higher intensity at the NGLCGS would permit much tighter collimation, thus enabling a vast improvement in beam energy resolution without an unacceptable reduction in beam intensity.

Nevertheless, the statistical error bars from $\mathrm{HI} \gamma \mathrm{S}$ shown in figure 26 are much lower than any previous experiment. By operating at a beam energy of $120 \mathrm{MeV}$ or higher, while staying below the pion production threshold, and using several very large, high-resolution NaI detectors with FWHM values of less than 2\% (as in the MAX-Lab experiment of references $[194,195])$ the new measurements would not only be more sensitive to neutron scalar polarizabilities, but would also provide much better control of the uncertainties associated with the inelastic channel (tables 7 and 8).

4.1.5.3. Neutron spin polarizabilities from elastic Compton scattering on polarized ${ }^{3} \mathrm{He}$. Polarized ${ }^{3} \mathrm{He}$ approximates a polarized neutron target. This statement can be quantified using $a b$ initio wave functions calculated with $\chi E F T$ potentials, see reference [185]. Such calculations also incorporate the meson-exchange currents that are needed for consistency with the 


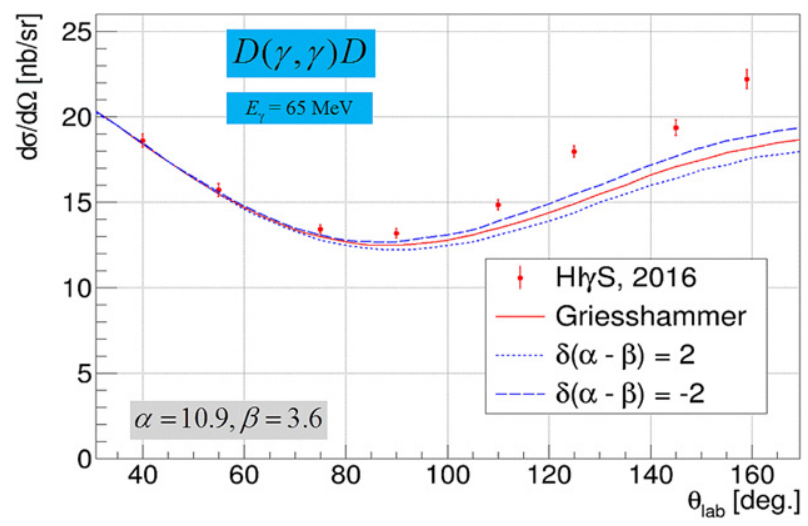

Figure 26. Differential cross section for elastic Compton scattering on deuterium at $65 \mathrm{MeV}$ as a function of laboratory angle. The curves are $\chi$ EFT calculations that have been varied by \pm 2 in the polarizability difference $\alpha_{E 1}^{(\mathrm{s})}-\beta_{M 1}^{(\mathrm{s})}[16,184]$. The agreement between data and theory is good at forward angles, but diverges at backward angles due to inelastic contributions.

Table 7. Suggested beam parameters for neutron scalar polarizabilities from deuteron.

\begin{tabular}{lc}
\hline Energy & $120 \mathrm{MeV}$ \\
Flux & $5 \times 10^{8}$ at $1.5 \%$ FWHM \\
Polarization & Circular \\
Diameter & $<20 \mathrm{~mm}$ on target \\
Time structure & TBD \\
\hline
\end{tabular}

Table 8. Suggested beam parameters for neutron spin polarizabilities from polarized ${ }^{3} \mathrm{He}$.

\begin{tabular}{lc}
\hline Parameter & Value \\
\hline Energy range & 100 to $140 \mathrm{MeV}$ \\
Flux on target & $5 \times 10^{8} \mathrm{\gamma} / \mathrm{s}$ \\
Relative energy resolution & $2 \% \mathrm{FWHM}$ \\
Polarization & Circular, polarization $>95 \%$ \\
Beam diameter & $5 \mathrm{~mm}$ on target \\
Time structure & $20 \mathrm{MHz}$, pulse width $<1 \mathrm{~ns}$ \\
\hline
\end{tabular}

potential. As ${ }^{3} \mathrm{He}$ is doubly charged and contains more nucleon pairs, interference of polarizability effects with proton Thomson terms and meson-exchange currents is larger than for the deuteron or proton, leading to some enhanced polarizability signals for this target.

The calculations depicted in figure 27 suggest that the photon beam asymmetry with a transversely polarized target, $\Sigma_{2 x}$, is the cleanest observable to determine a neutron spin polarizability. It is affected by $\gamma_{M 1 M 1}^{(\mathrm{n})}$ much more strongly than it is by the scalar polarizabilities, and it is unaffected by any proton spin polarizabilities. Other neutron spin polarizabilities affect this observable, but with a different angular dependence. Measuring this observable over a range of angles and energies will be crucial for checking the $\chi$ EFT theory of Compton scattering from ${ }^{3} \mathrm{He}$ and ensuring that 'contamination' of the polarization observable from scalar polarizabilities is understood and under control. 


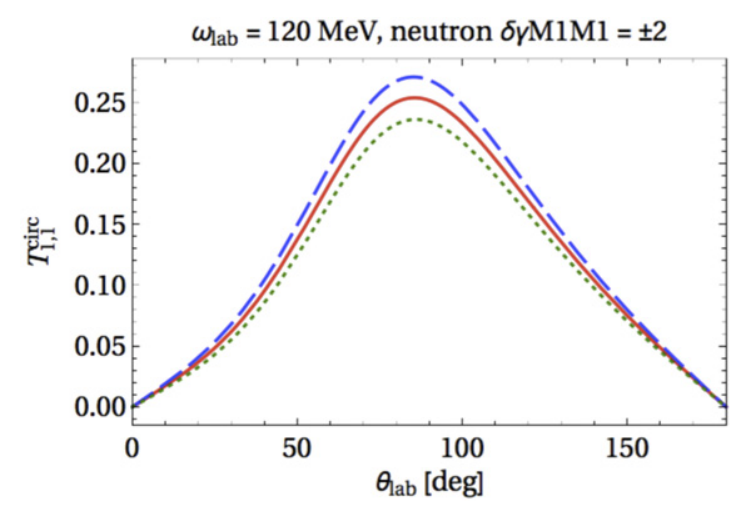

Figure 27. Predicted beam asymmetry for Compton scattering from a transversely polarized ${ }^{3} \mathrm{He}$ target at a photon laboratory energy of $120 \mathrm{MeV}$. The effect of varying $\gamma_{M 1 M 1}^{(\mathrm{n})}$ by 2 units is shown [185].

At $120 \mathrm{MeV}$, a similar sensitivity to neutron polarizabilities is predicted in the distribution of $\Sigma_{2 z}$, as measured with a longitudinally polarized target. In this case, a linear combination of four spin polarizabilities could be determined from the measurements.

A high-density polarized ${ }^{3} \mathrm{He}$ target has been built and tested at $\mathrm{HI} \gamma \mathrm{S}$ [199-201]. Given the demonstrated areal density of ${ }^{3} \mathrm{He}$, an experiment using the current HINDA detector array would collect on average ten elastic events per hour at each angle, assuming a beam intensity of $5 \times 10^{8} \mathrm{\gamma} / \mathrm{s}$. With a target polarization of $50 \%$, statistical uncertainties of order 0.02 on $\Sigma_{2 x}$ and $\Sigma_{2 z}$ could be achieved in moderate running times. Operation at such a high beam intensity would require continued development of an effective shielding configuration against backgrounds from the target windows. Additional development work is needed to improve the capability of the HINDA array to discriminate cosmic-ray events, which contribute at the $\gamma$-ray energies of interest in this experiment.

4.1.5.4. Neutron scalar polarizabilities from elastic Compton scattering on ${ }^{4} \mathrm{He} .{ }^{4} \mathrm{He}$ has a larger coherent Compton cross sections than any other nucleus discussed here, see figure 19 for the recent $\mathrm{HI} \gamma \mathrm{S}$ data on this reaction [202]. From a theoretical perspective, an accurate description of cross sections for ${ }^{4} \mathrm{He}$, which is more tightly bound than ${ }^{2} \mathrm{H}$ or ${ }^{3} \mathrm{He}$, as a function of energy and angle, will challenge the $\chi E F T$ description of Compton scattering on light nuclei. Thus, although measurements just below pion threshold hold the most promise for extractions of the neutron polarizabilities, ancillary measurements at lower energies are needed to check that the theory used for polarizability extractions is sufficiently accurate.

The higher flux and higher energy of the new facility mean that $\gamma^{4} \mathrm{He}$ elastic scattering could yield neutron scalar polarizabilities of unprecedented precision. The prospects are illustrated in figure 28, where the angular distributions are plotted for 60,90 and $120 \mathrm{MeV}$. We project that at $120 \mathrm{MeV}$ the beam parameters of table 9 , together with existing ${ }^{4} \mathrm{He}$ target technology (see section 4.1.6 below), and the present HINDA detector array, can produce a cross-section measurement with $1 \%$ statistical error in just $150 \mathrm{~h}$. This precision, combined with the estimated point-to-point systematic uncertainty ( $2 \%$ in solid angle) is depicted by the shaded bands in figure 28 . With the statistical uncertainties at such a reduced level, we expect that systematics (including an estimated $1 \%$ for target density and $2 \%$ for flux) and $\chi \mathrm{EFT}$ truncation uncertainty will ultimately limit the accuracy attained for $\alpha_{E 1}^{(\mathrm{n})}$ and $\beta_{M 1}^{(\mathrm{n})}$. Even so, this represents a significant advance in our knowledge of $\alpha_{E 1}^{(\mathrm{n})}$ and $\beta_{M 1}^{(\mathrm{n})}$. 


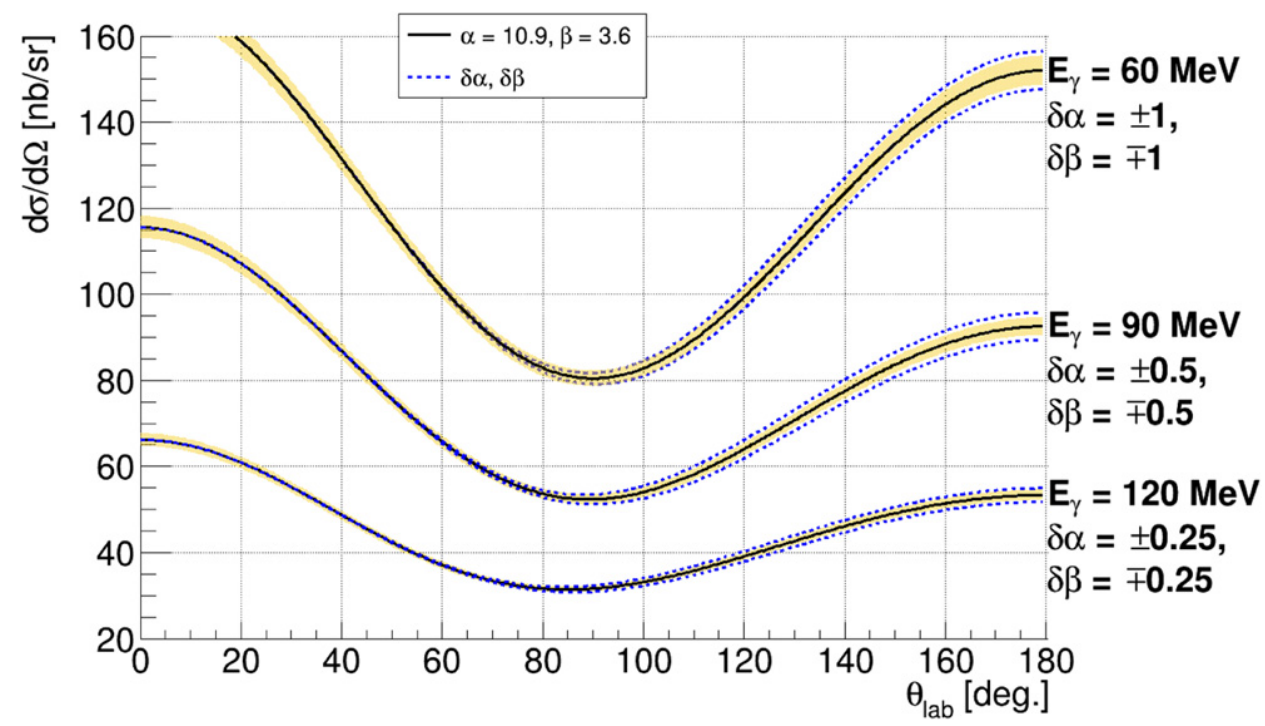

Figure 28. Predictions for the differential cross section for Compton scattering from ${ }^{4} \mathrm{He}$ at photon laboratory energies of 60,90 , and $120 \mathrm{MeV}$ in a phenomenological model [203]. The effect of varying the isoscalar $\alpha_{E 1}$ and $\beta_{M 1}$ while keeping their sum fixed is shown by the blue dotted lines. A combined statistical and point-to-point systematic uncertainty of $2.2 \%$ is indicated by the shaded bands.

Table 9. Suggested beam parameters for neutron scalar polarizabilities from ${ }^{4} \mathrm{He}$.

\begin{tabular}{lc}
\hline Parameter & Value \\
\hline Energy range & 60 to $120 \mathrm{MeV}$ \\
Flux on target & $10^{8}$ to $10^{9} \mathrm{\gamma} / \mathrm{s}$ \\
Relative energy resolution & $3 \% \mathrm{FWHM}$ \\
Polarization & None \\
Beam diameter & $5 \mathrm{~mm}$ on target \\
Time structure & Highest frequency available, pulse width $<1 \mathrm{~ns}$ \\
\hline
\end{tabular}

In addition to the cross-section advantage provided by a ${ }^{4} \mathrm{He}$ target (and also the obvious fact that the target is non-combustible), the energy resolution of the beam and detector become significantly less critical in this case, compared to deuterium. Since the first excited state of ${ }^{4} \mathrm{He}$ is near $20 \mathrm{MeV}$, it is possible to obtain very clean, purely elastic $\gamma$-ray spectra with a ${ }^{4} \mathrm{He}$ target. It is no longer necessary to impose tight beam collimation to achieve improved beam energy resolution, and greater photon fluxes on target are possible.

A measurement of coherent Compton scattering from ${ }^{3} \mathrm{He}$ at similar angles and energies will provide complementary information, since it is sensitive to a different combination of neutron and proton polarizabilities. While ${ }^{3} \mathrm{He}$ has a lower breakup threshold than ${ }^{4} \mathrm{He}$ and does not offer quite the same cross-section advantage, extractions of $\alpha_{E 1}^{(\mathrm{n})}-\beta_{M 1}^{(\mathrm{n})}$ from $\gamma^{3} \mathrm{He}$ scattering will, nevertheless, be a valuable cross-check that the theory used for polarizability extractions has nuclear effects under control. An experiment to measure the $\gamma-{ }^{3} \mathrm{He}$ elastic differential cross section is approved at $\mathrm{HI} \gamma \mathrm{S}$ and will run in 2019 at energies of $100 \mathrm{MeV}$ and (later) $120 \mathrm{MeV}$ [204]. The higher flux and extended energy range of the NGLCGS facility could provide enhanced sensitivity of this kind of ${ }^{3} \mathrm{He}$ measurement to neutron polarizabilities. 
4.1.5.5. Quasi-free neutron Compton scattering on light nuclei. Much of the experimental information we have on QF Compton scattering on light systems comes from the Mainz experiment of Kossert et al with an unpolarized photon beam and a deuterium target [205]. The experiment used tagged bremsstrahlung at energies of $E_{\gamma}=200$ to $400 \mathrm{MeV}$ and the large $\mathrm{NaI}(\mathrm{Tl})$ spectrometer 'CATS' to detect the backscattered photon at $136^{\circ}$. In addition, the 'SENECA' array of liquid scintillators was used to detect the recoiling neutron (or proton). The cross section peaks at around $180 \mathrm{nb} / \mathrm{sr}$ at the $\Delta$ resonance. It rises from about 40 $\mathrm{nb} / \mathrm{sr}$ to around $150 \mathrm{nb} / \mathrm{sr}$ as $E_{\gamma}$ increases from 250 to $300 \mathrm{MeV}$, so there is definite benefit if one could run at around $300 \mathrm{MeV}$. A QF neutron Compton experiment at these energies with a polarized beam and a polarized ${ }^{3} \mathrm{He}$ target would provide information on neutron dynamical spin polarizabilities in this energy regime. This would provide important constraints on these dynamical neutron spin-structure functions in this regime where the $\Delta(1232)$ and pioncloud mechanisms both affect pion-production multipoles - constraints which would facilitate enhanced precision for the static values of the polarizabilities.

We note that $\Sigma_{2 x}$ for Compton scattering from a neutron is mainly sensitive to $\gamma_{E 1 E 1}^{(\mathrm{n})}$, especially at forward angles, as seen in figure $29[182,183]$. The sensitivity to $\alpha_{E 1}^{(\mathrm{n})}-\beta_{M 1}^{(\mathrm{n})}$ here is quite small. The kinematics of QF Compton scattering from deuterium at an incident energy of $300 \mathrm{MeV}$ is displayed in figure 30 .

With a $10 \mathrm{~cm}$ LD target, a $1 \mathrm{sr}$ detector for $\gamma^{\prime}$ and a photon beam intensity of $10^{9} \gamma / \mathrm{s}$, the Compton event rate would be about $70 \mathrm{~Hz}$. Such a high rate of incident $\gamma$-rays also makes the use of a polarized ${ }^{3} \mathrm{He}$ gas target feasible. A $30 \mathrm{~cm}, 10$ bar target with a similar detector acceptance would produce an event rate of about $1.2 \mathrm{~Hz}$. Given that the neutron angle is fairly tightly correlated to the $\gamma^{\prime}$ angle it should be possible to design the neutron arm of the detection system to match the acceptance of the $\gamma^{\prime}$-arm.

Neutron spin polarizabilities could be accessed using two different implementations of double-polarization: either polarized beam, polarized target and TOF neutron detector or polarized beam, unpolarized target, and a TOF polarimeter to measure the polarization transfer to the recoiling neutron.

Typically, in a polarized experiment one measures an asymmetry in the counting rate when the beam helicity, target spin orientation, etc are reversed. The precision in measuring a polarized observable is given by: $\delta X=\sqrt{\frac{2}{N_{\text {inc }} \cdot F^{2}}}$, where $N_{\text {inc }}$ is the number of incident neutrons and $F^{2}$ is the experiment FOM. If the efficiency of the $\gamma^{\prime}$ arm is close to $100 \%, F^{2} \sim \varepsilon_{n} P^{2}$, where $\varepsilon_{n}$ is the efficiency of the neutron arm and $P$ is either the target polarization or the analyzing power of the neutron polarimeter. In the polarized-beam-and-target approach, $\varepsilon_{n} \sim 0.2$ and $P \sim 0.9 \times 0.6$ are readily obtainable, so that $F^{2} \sim 5.8 \times 10^{-2}$. To obtain a precision of $\delta X \sim 0.01, N_{\text {inc }}$ should be about $3 \times 10^{5}$, which could be collected in $80 \mathrm{~h}$.

We prefer this option to the one where the polarization of the recoiling neutron is detected. The precision of a recoil-polarization detection experiment for a given $N_{\text {inc }}$ could be similar, even though the FOM is lower, since a liquid target could be employed. But other complications regarding the detection of final-state neutron polarization-imprecise knowledge of the neutron analyzing power, complications in building a polarimeter that matches the solid angle of the $\gamma^{\prime}$ arm-favor a polarized-target experiment. But recoil-polarization detection should continue to be considered as an option for NGLCGS experiments.

4.1.6. Target technology. History shows that polarizability measurements proceed at exactly the pace of the enabling technologies. It is telling that the first spin polarizability measurements followed the construction of a low-mass, high acceptance, frozen-spin target at Mainz, 


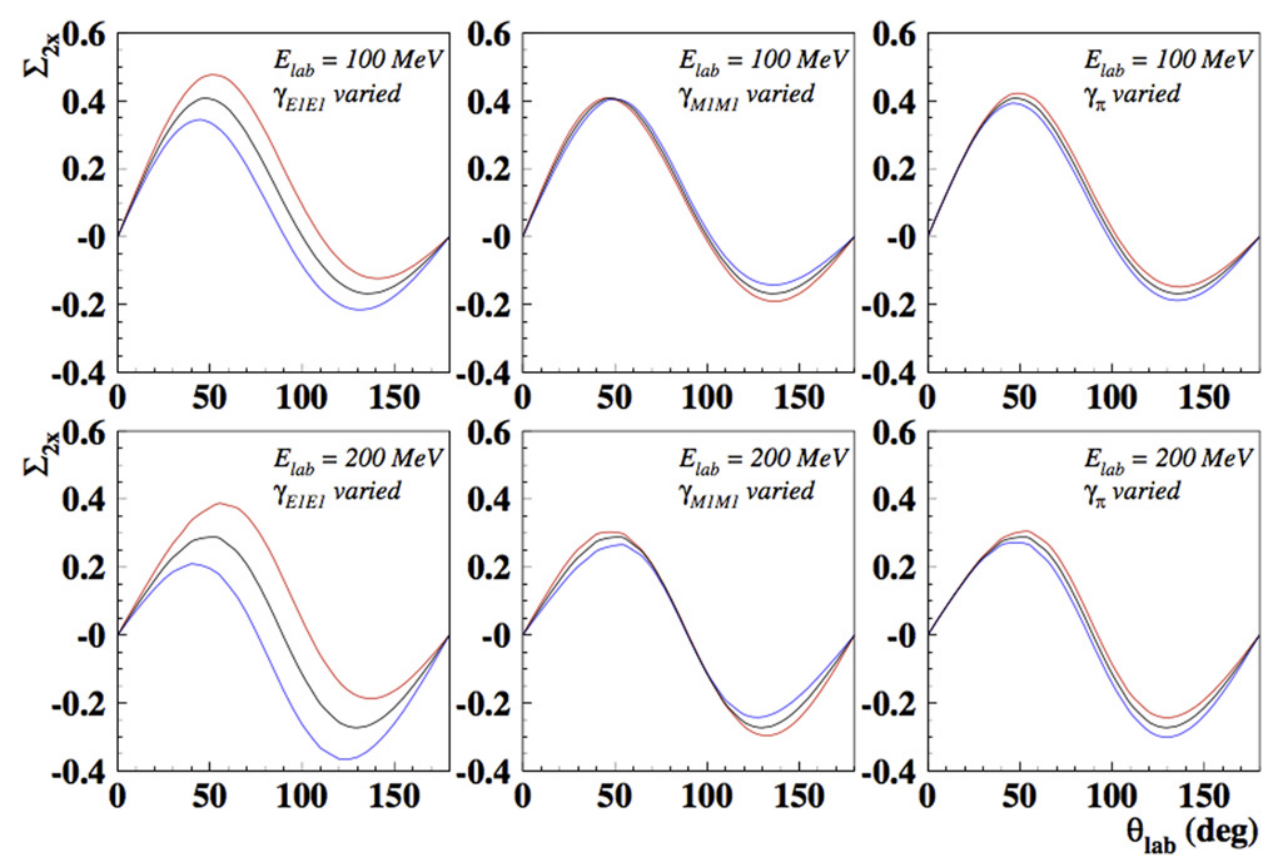

Figure 29. Predicted beam-target asymmetry for Compton scattering from a transversely polarized neutron at photon laboratory energies of $100 \mathrm{MeV}$ (upper row), and $200 \mathrm{MeV}$ (lower row) [183]. The results from subtracted dispersion relations are obtained by using the values $\alpha_{E 1}^{(\mathrm{n})}=12.5, \beta_{M 1}^{(\mathrm{n})}=2.7$ and $\gamma_{0}^{(\mathrm{n})}=-0.096$, while the remaining polarizabilities are taken as free parameters. Left column: results for fixed $\gamma_{M 1 M 1}^{(\mathrm{n})}$ and $\gamma_{\pi}^{(\mathrm{n})}$, and the following values of $\gamma_{E 1 E 1}^{(\mathrm{n})}:-5.94$ (black lines), -3.94 (red lines), and -7.94 (blue lines); central column: results for fixed $\gamma_{E 1 E 1}^{(\mathrm{n})}$ and $\gamma_{\pi}^{(\mathrm{n})}$, and the following values of $\gamma_{M 1 M 1}^{(\mathrm{n})}: 3.75$ (black lines), 5.75 (red lines), and 1.75 (blue lines); right column: results for fixed $\gamma_{E 1 E 1}^{(\mathrm{n})}$ and $\gamma_{M 1 M 1}^{(\mathrm{n})}$, and the following values of $\gamma_{\pi}^{(\mathrm{n})}: 13.68$ (black lines), 15.68 (red lines), and 11.68 (blue lines).

and that to drive this research further, a further extension of this device, an active polarized target, has been developed. In addition, without the construction of a modern, high-rate, highly segmented photon detector, covering a large fraction of the $4 \pi$ solid angle, it will not be possible to suppress pion photoproduction backgrounds.

4.1.6.1. Unpolarized active gas targets. In unpolarized active gas targets, a high pressure gas scintillator of ${ }^{3} \mathrm{He}$ or ${ }^{4} \mathrm{He}$ is generally employed with an admixture of an inert gas to shift the primary VUV scintillation into the visible region for easier detection. Xenon is a commonly used shifter, but alternatively one can use nitrogen, which gives good results at low concentrations of around $500 \mathrm{ppm}$. The scintillation at $20 \mathrm{bar}$ and $500 \mathrm{ppm} \mathrm{N}_{2}$ is quite fast, with rise and fall times of a few nanoseconds. This provides good timing information and allows operation at fairly high rates. Keeping the amount of high- $Z$ material in the target to a minimum reduces the sensitivity to pair production and (electron) Compton scattering by a high intensity incident photon beam.

The active target allows the recoiling charged particle from a Compton event (coherent or QF) to be detected in coincidence with the scattered photon. This helps separate the weak signal from background. Generally a gas target would be fairly long in order to achieve a reasonable 

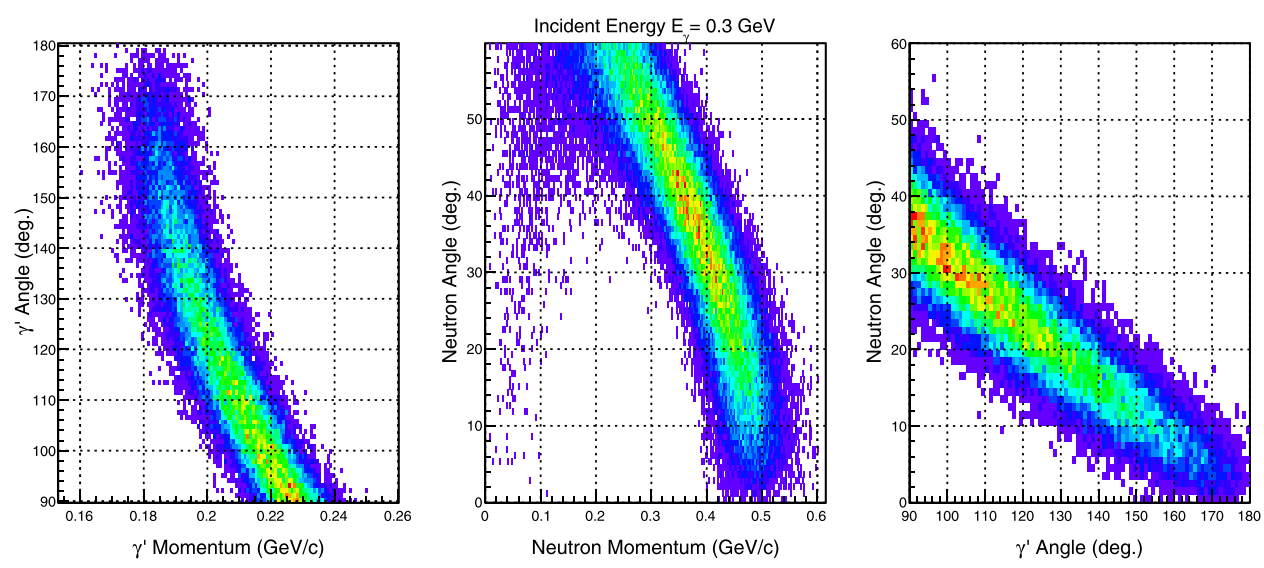

Figure 30. QF kinematics at $300 \mathrm{MeV}$ incident energy.
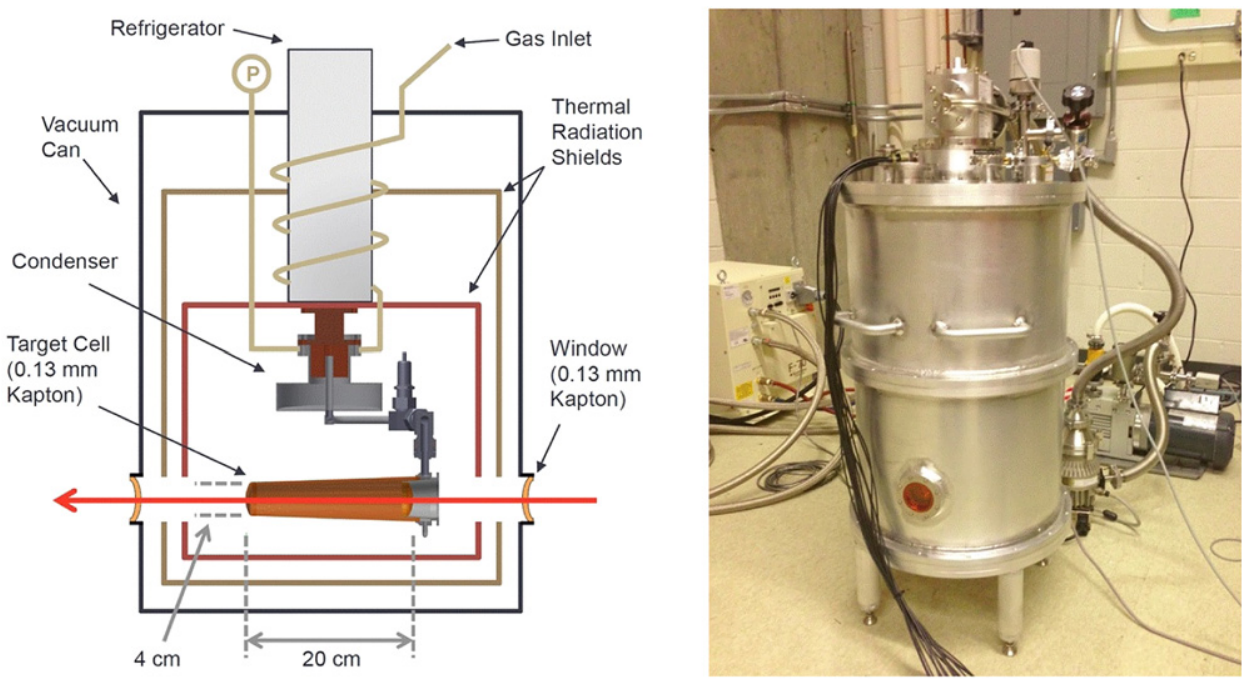

Figure 31. The cryotarget at $\mathrm{HI} \gamma \mathrm{S}$ [206].

thickness, so even fairly crude vertex reconstruction is advantageous in measuring angular distributions.

4.1.6.2. Unpolarized liquid targets. Liquid ${ }^{3} \mathrm{He}$ and ${ }^{4} \mathrm{He}$ targets are relatively standard technology. The liquid has a density about a factor of fifty greater than a 20 bar gas target. A cryogenic target system capable of liquefying ${ }^{4} \mathrm{He}$ at $4 \mathrm{~K}, \mathrm{H}_{2}$ at $20 \mathrm{~K}$, and $\mathrm{D}_{2}$ at $23 \mathrm{~K}$ has already been developed at $\mathrm{HI} \gamma \mathrm{S}$ [206]; see figure 31. The apparatus was utilized in a series of Compton scattering experiments on deuterium and ${ }^{4} \mathrm{He}$ at beam energies from 60 to $85 \mathrm{MeV}$. Cooling is provided by a $4 \mathrm{~K}$ Gifford-McMahon cryocooler. Liquid temperatures and condenser pressures are recorded throughout each run to ensure that the target's areal density is known to about $1 \%$. Modifications are planned that will add the ability to liquefy ${ }^{3} \mathrm{He}$ by lowering the system's base temperature to $1.5 \mathrm{~K}$. 
Elastic Compton scattering measurements on liquid ${ }^{4} \mathrm{He}$ and deuterium targets at the NGLCGS will require a high-resolution photon detector array to identify elastic scattering unambiguously. In the case of ${ }^{4} \mathrm{He}$, the requirement is not especially stringent, since the inelastic channel is about $20 \mathrm{MeV}$ below the elastic peak. The situation is considerably more challenging for deuterium targets, where the breakup channel is only $2.2 \mathrm{MeV}$ below the elastic peak. A program is underway at $\mathrm{HI} \gamma \mathrm{S}$ using the $\mathrm{LD}$ target with high-resolution beams and detectors to characterize the inelastic contribution at 65 and $85 \mathrm{MeV}$.

Both liquid ${ }^{3} \mathrm{He}$ and ${ }^{4} \mathrm{He}$ could also be used for QF Compton scattering, but the recoiling nucleon, and perhaps other fragments, would need to be detected in coincidence with the scattered photon. Time of flight to an external array could be used to measure the momentum of the recoil neutron. This would be very useful in reconstructing the QF event. If the energy is sufficiently high, similar techniques could be preformed for a recoiling proton. At lower energies a separate, active target could detect protons stopping in the target. The technique of using TOF for both neutrons and protons simultaneously could provide a tremendous advantage in the particular case of QF scattering from a deuterium target, where the proton QF measurement could provide a consistency check for the desired neutron QF result, since the two QF channels are completely symmetric.

4.1.6.3. Frozen-spin polarized target. The proton spin-polarizability program depends critically on developing a proton target that can be polarized in either the longitudinal or the transverse directions with high polarization and a long relaxation time. As an example, the Mainz A2 group, in collaboration with JINR Dubna, has constructed a frozen-spin butanol target ideally suited for these measurements [207].

The workhorse of the frozen-spin target is a horizontal DR with high cooling power designed for insertion into a nearly $4 \pi$ detector. Initially, the target end of the cryostat is placed inside the warm bore of a large superconducting magnet. The target material consists of frozen beads made from butanol which has been doped with the stable free nitroxyl radical TEMPO $(2,2,6,6$ tetramethyl-piperidine-1-oxyl). The beads reside in the DR mixing chamber, which is cooled below $1 \mathrm{~K}$. The electrons are readily polarized at low temperature in a $2.5 \mathrm{~T}$ magnetic field. Their polarization is then transferred to the protons via dynamic nuclear polarization using $70 \mathrm{GHz}$ microwaves. After polarization, the microwaves are switched off and their attendant heat load is removed. The target then cools to a base temperature of around $30 \mathrm{mK}$, and a $0.6 \mathrm{~T}$ holding field (provided by a small superconducting coil inside the cryostat) maintains the polarization. The bulky polarizing magnet can be removed, and the target inserted into a large-acceptance detector. The Mainz frozen-spin target achieves a proton polarization of about $90 \%$ with relaxation times greater than about $1000 \mathrm{~h}$. Longitudinal or transverse directions are both available, depending upon how the internal holding coil is wound (solenoid vs saddle). Deuterated butanol may be substituted as the target material with deuteron vector and tensor polarizations in excess of $75 \%$ and $40 \%$, respectively.

4.1.6.4. Active frozen-spin target. As discussed in the physics justification section, there is great interest in making measurements of the proton spin polarizabilities at incident photon energies near pion threshold. However, at incident photon energies below approximately $250 \mathrm{MeV}$, recoil protons cannot escape from a frozen-spin target cryostat, and the identification of Compton scattering events from the sea of $\pi^{0} \rightarrow \gamma \gamma$ events becomes problematic. At Mainz, for example, the direction of the recoil proton is used to differentiate Compton scattering events from $\pi^{0}$ decay events. To enable Compton scattering measurements at lower energies, where the recoil proton is presently unobserved, an active frozen-spin target has been developed. The target material is a polarizable scintillator, employing Si photomultipliers to read out the scintillation. A similar active polarized target will be essential for this program. 

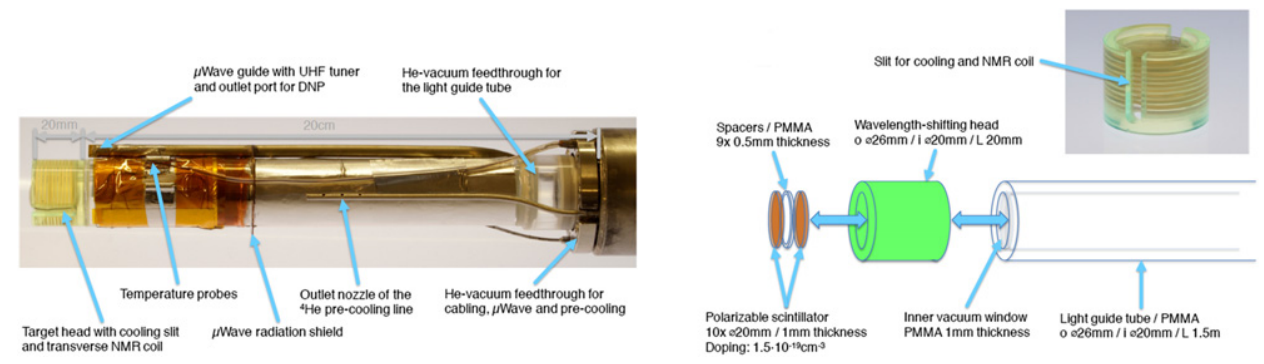

Figure 32. The Mainz active polarized target [210].

An active frozen-spin target utilizes the same ${ }^{3} \mathrm{He} /{ }^{4} \mathrm{He}$ DR as a traditional frozen-spin target, with some modifications in and around the area that holds the target material. Discs of polystyrene doped with TEMPO are stacked inside the DR mixing chamber and held at 30 $\mathrm{mK}$. Scintillation light from the discs is captured, redirected, and wavelength-shifted in a containing cylinder of wavelength-shifting material [208]. This cylinder is, in turn, affixed to a cylinder of plexiglass, which transports the light to silicon photomultipliers operating at $4 \mathrm{~K}$ [209] (see figure 32). In a recent test at Mainz, the active target achieved 50\% polarization with a $70 \mathrm{~h}$ relaxation time. A target head which enables particle tracking is also under development. This could involve the use of printable scintillators in order to make a highly segmented, position-sensitive target head.

4.1.6.5. Polarized ${ }^{3} \mathrm{He}$ gas targets. Polarized ${ }^{3} \mathrm{He}$ is an effective polarized neutron target, as about $90 \%$ of the nuclear spin can be attributed to the unpaired neutron. It is by now a quite mature, though far from trivial, technology. The technique of spin-exchange optical pumping is used to achieve high-pressure $(\approx 10 \mathrm{~atm})$ and high-polarization $(\approx 70 \%)^{3} \mathrm{He}$ targets. First, a narrow-band circularly polarized laser at a wavelength of $795 \mathrm{~nm}$ hitting rubidium vapor quickly polarizes those atoms. The rubidium atoms then collide with ${ }^{3} \mathrm{He}$ atoms and transfer their polarization to the ${ }^{3} \mathrm{He}$ nuclei. The rubidium and ${ }^{3} \mathrm{He}$ mixture is maintained at about 10 atm in a glass pumping cell transparent to laser light and at a temperature $\approx 200{ }^{\circ} \mathrm{C}$ so that rubidium stays in its gaseous state. This spherical pumping cell is connected to a cylindrical target cell where the beam window made as thin as possible, so as to minimize photon interactions in the glass. Special glass that is inert to the alkali vapor and stable in this radiation environment must be used.

The whole target system is maintained in a very homogeneous magnetic field to ensure a long polarization lifetime. Adiabatic fast passage nuclear magnetic resonance (AFP-NMR) and electron paramagnetic resonance are used to measure the ${ }^{3} \mathrm{He}$ polarization. AFP-NMR is also used to flip the direction of polarization to reduce systematic uncertainties for asymmetry measurements.

A polarized ${ }^{3} \mathrm{He}$ target of this type was developed for JLab [211] and achieved a world record $60 \%$ polarization with an incident $6 \mathrm{GeV}$ luminosity of $10^{36} /\left(\mathrm{cm}^{2} \mathrm{~s}\right)$. Meanwhile, the polarized ${ }^{3} \mathrm{He}$ target system at $\mathrm{HI} \gamma \mathrm{S}$ has reached $50 \%$ polarization and been used successfully in several experiments [200, 212, 213]. The luminosities proposed for the NGLCGS will not be an issue for this technology. R \& D on these targets is thus focused on enhancing signals with higher gas density and on reducing background from the glass cells. Note that the presence of the highly reactive alkali vapor inside the target mitigates against any sort of active target-either scintillator or drift-chamber variant. 


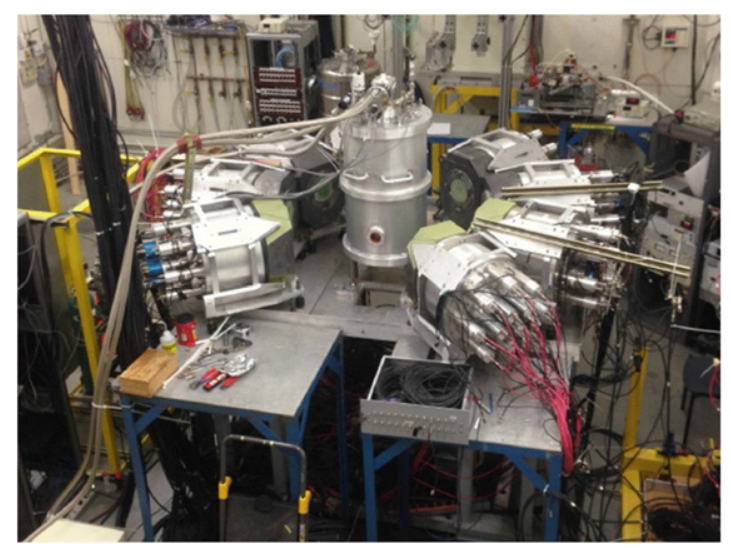

Figure 33. The high intensity gamma source $\mathrm{NaI}$ detector array HINDA with eight $\mathrm{NaI}$ crystals at $\mathrm{HI} \gamma \mathrm{S}$ is an example of present-day inorganic scintillators.

4.1.7. Detectors. An essential part of the experimental apparatus shared by many of the Compton scattering polarizability experiments is a nearly $4 \pi$ crystal detector for detection of the Compton-scattered photon. Near- $4 \pi$ acceptance is desirable for two reasons: (i) to enable data taking at multiple scattering angles simultaneously, and (ii) to suppress $\pi^{0} \rightarrow \gamma \gamma$ events that would show up as double neutral hits in the detector. Surrounding the target with a chargedparticle tracker-e.g., a cylindrical GEM or silicon tracker-would provide neutral/charged particle identification. For example, the tracking detector could be used to identify recoil protons, deuterons or helium nuclei from the Compton scattering reaction.

For the case of measuring the neutron polarizabilities, detectors suitable for photons or neutrons of energy around $100 \mathrm{MeV}$ will be necessary. Good detection efficiency of neutral particles requires bulk material, and scintillators are generally used.

4.1.7.1. Inorganic scintillators for photon detection. For photons one would use high- $Z$ materials which have short radiation lengths. Since energy resolution will be important, scintillators are preferable to Cherenkov counters. Traditionally materials such as $\mathrm{NaI}(\mathrm{Tl})$ or $\mathrm{CsI}(\mathrm{Tl})$ have been used, like in HINDA at $\mathrm{HI} \gamma \mathrm{S}$; see figure 33. However, (with thallium activation to increase light output) the scintillation is relatively slow and timing precision for TOF measurements is sub-optimal. Alternatively one can use materials such as:

(a) $\mathrm{BaF}_{2}$ : this has fast and slow scintillation components, with good timing properties and pulse shape discrimination (PSD) of relativistic and non-relativistic particles. The UV scintillation requires a quartz window photo-multiplier tube (PMT).

(b) $\mathrm{PbWO}_{4}$ : this material has quite a fast scintillation, good timing, short radiation length, and good radiation hardness. If we have a very high intensity facility, radiation hardness may become an issue in the long term.

(c) New developments in scintillator materials technology, such as the use of quantum dots. These aim to improve the light output and speed of the scintillation, and significant advances can be expected in the next few years.

These materials are sensitive to $\gamma$-rays and neutrons above about $25 \mathrm{MeV}$ as well as other generated background. For operation in intense radiation fields, the detectors should be highly segmented to keep singles rates at a manageable level. Uniformity of scintillation light collection is important if optimum energy resolution is to be obtained. 
If the scintillation is in the visible region one can use silicon PMTs in place of the traditional vacuum tubes. These are relatively cheap, have very good timing properties, reasonable noise performance, and continue to improve.

4.1.7.2. Organic scintillators. Organic scintillators are traditionally used for neutrons with energies of one to a few hundred $\mathrm{MeV}$. They are also sensitive to photons, but because of their long radiation lengths, they do not have good photon energy resolution. In general they produce fast scintillations so that they are suitable for high resolution TOF measurement, which is the only way practical way to achieve some energy resolution for neutrons of around $100 \mathrm{MeV}$. Organic scintillators come in two types:

- Plastic: plastic scintillators are relatively cheap and easy to fashion into a variety of geometries. In general they do not have PSD properties, although Eljen Technology does claim PSD for one of their variants. The hydrogen-to-carbon ratio in these scintillators is generally around $1: 1$

- Liquid: liquid scintillators are also cheap but obviously require a container. Traditional types similar to NE213 have good PSD properties but suffer from a volatile, chemically reactive base. Mineral-oil types have varying degrees of PSD, are fast, easily contained, and some have a hydrogen-to-carbon ratio as high as $2: 1$.

4.1.7.3. Time-of-flight techniques. Typical experimental setups for TOF measurements depend on fast timing, relatively long flight paths (for good timing resolution), and segmented detector arrays (for position information). Previous TOF setups have included multi-cell liquidscintillator arrays as well as large plastic scintillator bars that use time differences between the ends of each bar to pin down the lateral position of the neutron event. Given the necessity of a long flight path, these arrays generally occupy a considerable area, in order to guarantee a reasonable solid-angle acceptance for the neutron arm.

\subsection{QCD origins of charge symmetry breaking}

The quark masses are important input parameters in the SM. They are currently determined from a variety of sources, including meson masses and lattice simulations. The currently accepted values (in the $\overline{\mathrm{MS}}$ scheme at a renormalization scale of $2 \mathrm{GeV}$ ) are [214]:

$$
m_{u} \approx 2.2_{-0.4}^{+0.6} \mathrm{MeV} \text { and } m_{d} \approx 4.7_{-0.4}^{+0.5} \mathrm{MeV}
$$

This implies $m_{d} / m_{u} \geqslant 2$, which might naively imply large isospin-violation effects. These effects are, however, efficiently masked, since chiral symmetry means that all quark-mass effects are generically suppressed in low-energy strong-interaction observables.

Furthermore, for reactions involving only pions, effects proportional to a single power of $m_{u}-m_{d}$ are forbidden, due to G-parity. However, the presence of nucleons vitiates this theorem, and isospin violation should thus be a stronger effect in meson-nucleon interactions. This provides an important opportunity for a next-generation laser Compton $\gamma$-ray source (NGLCGS) to contribute to the determination of a fundamental parameter of the SM, $m_{d} / m_{u}$.

To be specific, pion photoproduction offers two ways of observing effects due to isospin violation. The first consists of a precise measurement of the four pion photoproduction s-wave amplitudes: $\gamma p \rightarrow \pi^{+} n, \gamma p \rightarrow \pi^{0} p, \gamma n \rightarrow \pi^{-} p$, and $\gamma n \rightarrow \pi^{0} n$. (The latter two cases would be observed via coherent production from the deuteron.) A recent TRIUMF experiment, experiment E643, attempted to extract the s-wave amplitude for the process $\gamma n \rightarrow \pi^{-} p$ from the measurement of the total and differential cross sections of the inverse reaction $\pi^{-} p \rightarrow \gamma n$. To be quantitative, the s-wave amplitude for the charged-particle channels should be determined 
to within an accuracy of $1 \%$, and for the neutral channels to within $5 \%$. Accurate predictions for all four channels exist [215] making use of the conventional isospin symmetric basis of three independent amplitudes. The theoretical framework of consistently including operators related to the quark-mass difference and to virtual photons is currently being developed [216]. A determination of the (absolute) total cross section for the charged channels with a $2 \%$ accuracy appears to be sufficient to provide an important check on the quark-mass ratio $m_{d} / m_{u}$ extracted from mesonic processes.

Recently, there have been two independent claims that isospin has been violated at about the $7 \%$ level in medium energy $\pi N$ scattering [217]. As pointed out by Bernstein [218], a very precise determination of the phase of $\gamma p \rightarrow \pi^{0} p$ below the secondary $\pi^{+} n$ threshold would allow for a determination of the s-wave $\pi N$ scattering length $a_{\pi N}\left(\pi^{0} p\right)$ via a generalized three-channel Fermi-Watson analysis. As shown by Weinberg [219], the difference $a_{\pi N}\left(\pi^{0} p\right)-a_{\pi N}\left(\pi^{0} n\right)$ is very sensitive to the quark-mass difference $m_{u}-m_{d}$. A measurement of $\operatorname{Im}\left(E_{0+}\right)$ is equivalent to a measurement of the corresponding $\pi N$ phase shift. It is important to map out this quantity in the region of the so-called unitary cusp (150-170 MeV) [218]: the discontinuity in $E_{0+}$ which results from the fact that the $\pi^{0} p$ and $\pi^{+} n$ thresholds are different (a result of isospin breaking). A measurement of $\operatorname{Im}\left(E_{0+}\right)$ on a polarized proton target using the intensity and energy resolution of the NGLCGS will yield an accuracy that easily displays isospin violation if it is present at the level claimed above.

More generally, pion-nucleon scattering is poised for significant advances due to the advent of Roy-Steiner equations that incorporate the consequences of chiral symmetry. Threshold pion photoproduction can be used to measure phase shifts at low energies in charge states that cannot be reached in $\pi N$ scattering. This involves measuring $\gamma p \rightarrow \pi^{0} p, \pi^{+} n$ and $\gamma n \rightarrow$ $\pi^{-} p, \pi^{0} n$ reactions.

In the two-nucleon sector the difference between the neutron-neutron and proton-proton scattering lengths is one of the strongest signals of charge-symmetry breaking in few-nucleon systems. But $a_{\mathrm{nn}}$ is clouded in controversy due to conflicting values from the reactions $\pi^{-} d \rightarrow$ $\gamma n n$ and $n d \rightarrow p n n$. The reaction $\gamma d \rightarrow \pi^{+} n n$ provides an alternative avenue for a measurement of $a_{\mathrm{nn}}$. The NGLCGS would have the intensity and energy resolution to be well-suited for this measurement and therefore shed light on this important quantity.

\section{Accelerator concepts of next generation laser Compton gamma-ray beam facilities}

\subsection{Introduction to Compton gamma-ray sources}

Since the discovery of the Compton effect in the early 1920s via the scattering of x-rays from electrons in metals [220, 221], another four decades would pass before the Compton effect was recognized as a useful mechanism to convert low energy photons to high energy x-ray and $\gamma$-ray photons. With the development and operation of charged particle accelerators with relativistic electron beams, in 1963, Milburn and, independently, Arutyunian and Tumanian proposed a method of producing very high energy $\gamma$-ray beams via Compton back-scattering of photons from high energy electrons [222, 223]. In the ensuing years, the first experimental demonstrations of high energy $\gamma$-ray production using Compton scattering were carried out by several groups around the world, including Kulikov et al with a $600 \mathrm{MeV}$ synchrotron [224], Bemporad et al with the $6.0 \mathrm{GeV}$ Cambridge electron accelerator [225], and Ballam et al with the $20 \mathrm{GeV}$ Stanford linear accelerator [226, 227].

While successful, the first demonstrations of $\gamma$-ray production by Kulikov et al [224] and Bemporad et al [225] had a very low $\gamma$-photon yield. With much improved photon yields 
Table 10. A list of laser Compton CGSs around the world which are either operational or being developed for operation in the near future.

\begin{tabular}{|c|c|c|c|c|c|c|c|}
\hline Project name/parameters & HIGS & LEPS/LEPS2 & NewSUBARU & UVSOR-III & SLEGS & XGLS & ELI-NP \\
\hline Location & Durham, US & Hyogo, Japan & Hyogo, Japan & Okazaki, Japan & Shanghai, China & Xi'an, China & $\begin{array}{c}\text { Bucharest-Magurele, } \\
\text { Romania }\end{array}$ \\
\hline Accelerator technology & Storage ring & Storage ring & Storage ring & Storage ring & Storage ring & Linac & Storage ring \\
\hline Laser technology & FEL & $\begin{array}{l}\text { Solid state } \\
\text { laser }\end{array}$ & $\begin{array}{c}\text { Solid state/gas } \\
\text { laser }\end{array}$ & Fiber/gas laser & $\mathrm{CO}_{2}$ laser & Ti:sapphire laser & Solid state laser \\
\hline Collision technology & $\begin{array}{l}\text { Intra-cavity, } \\
\text { head-on }\end{array}$ & $\begin{array}{l}\text { External laser, } \\
\text { head-on }\end{array}$ & $\begin{array}{l}\text { External laser, } \\
\text { head-on }\end{array}$ & $\begin{array}{l}\text { External laser, } \\
\text { head-on }\end{array}$ & $\begin{array}{l}\text { External laser, } \\
\text { cross-angle/head-on }\end{array}$ & $\begin{array}{l}\text { External laser, } \\
\text { head-on }\end{array}$ & $\begin{array}{l}\text { Intra-cavity, } \\
\text { head-on }\end{array}$ \\
\hline Electron energy $(\mathrm{MeV})$ & $240-1200$ & 8000 & $500-1500$ & 750 & 3500 & $120-360$ & $234-742$ \\
\hline Laser wavelength (nm) & $1060-190$ & 266 and 355 & $532-10600$ & $\begin{array}{c}1940 \\
\text { and } 10600\end{array}$ & 10640 & 800 & $1030 / 515$ \\
\hline \multicolumn{8}{|l|}{ Charge and temporal structure } \\
\hline $\begin{array}{l}\text { A. CW: Avg. current }(\mathrm{mA}), \\
Q(\mathrm{nC}) @ \text { reprate }(\mathrm{MHz})\end{array}$ & $\begin{array}{c}10-120, \\
1.8-22 @ 5.58\end{array}$ & $\begin{array}{c}100 \\
0.2-2 @ 50-500\end{array}$ & $\begin{array}{c}300, \\
0.6 @ 500\end{array}$ & $\begin{array}{c}300 \\
3 @ 90\end{array}$ & $\begin{array}{c}100-300, \\
0.28-0.87 @ 347\end{array}$ & & $1 @ 71.4$ \\
\hline $\begin{array}{l}\text { B. Pulsed: } f_{\mathrm{RF}}(\mathrm{MHz}), \\
\text { pulse: } Q(\mathrm{nC}) @ \text { reprate }(\mathrm{Hz}), \\
\text { pulse duration (full-width) }\end{array}$ & & & & & & $\begin{array}{l}2856 \text { (s-band), } \\
0.5 @ 10,10 \mathrm{ps} \\
\text { (micropulses) }\end{array}$ & \\
\hline$\gamma$-beam energy $(\mathrm{MeV})$ & $1-100$ & $1300-2900$ & $1-40$ & $1-5.4$ & $0.4-20$ & $0.2-3$ & $1-19.5$ \\
\hline Polarization & Lin, Cir & Lin, Cir & Lin, Cir & Lin, Cir & Lin, Cir & Lin, Cir & Lin \\
\hline$\gamma$-beam energy resolution & $0.8 \%-10 \%$ & $<15 \%$ & $10 \%$ & $2.9 \%$ & $<5 \%$ & $1.2 \%-10 \%$ & $<0.5 \%$ \\
\hline (FWHM) & collimation & tagging & $\begin{array}{l}(\phi=3 \mathrm{~mm}) \\
\text { collimation }\end{array}$ & $\begin{array}{l}(\phi=2 \mathrm{~mm}) \\
\text { collimation }\end{array}$ & $\begin{array}{l}(\phi=2 \mathrm{~mm}) \\
\text { collimation }\end{array}$ & collimation & collimation \\
\hline \multicolumn{8}{|l|}{$\gamma$-beam temporal structure } \\
\hline A. $\mathrm{CW}$ operation $(\mathrm{MHz})$ & 5.58 (typical) & $50-500$ & 500 & 90 & 347 & & 71.4 \\
\hline B. Pulsed operation & $\begin{array}{c}0.5-1.5 \mathrm{~ms}(\mathrm{FW}) \\
2-100 \mathrm{~Hz} \\
\text { gain modulated }\end{array}$ & & $\begin{array}{l}8 \mathrm{~ns} \text { pulse, } \\
10-100 \mathrm{kHz}, \\
Q \text {-switched } \\
\text { lasers }\end{array}$ & & & pulsed, $10 \mathrm{~Hz}$ & \\
\hline
\end{tabular}


Table 10. continued

\begin{tabular}{|c|c|c|c|c|c|c|c|}
\hline On-target flux (avg, $\gamma / \mathrm{s}$ ) & $10^{3}-3 \times 10^{9}$ & $10^{6}-10^{7}$ & $\begin{array}{l}10^{5}-3 \times 10^{6} \\
(\phi=3 \mathrm{~mm})\end{array}$ & $\begin{array}{c}4 \times 10^{5} \\
(\phi=2 \mathrm{~mm})\end{array}$ & $\begin{array}{c}10^{5}-10^{7} \\
(\phi=2 \mathrm{~mm})\end{array}$ & $10^{6}-10^{8}$ & $\sim 10^{8}$ \\
\hline Total flux (avg, $\gamma / \mathrm{s})$ & $10^{6}-3 \times 10^{10}$ & $10^{6}-10^{7}$ & $10^{7}-4 \times 10^{7}$ & $10^{7}$ & $10^{6}-10^{8}$ & $10^{8}-10^{9}$ & $10^{11}$ \\
\hline $\begin{array}{l}\text { Operation status or } \\
\text { projected operation date }\end{array}$ & Since 1996 & Since 1999 & Since 2005 & Since 2015 & $\begin{array}{l}\text { Under construction, } \\
\text { Operation in } 2022\end{array}$ & $\begin{array}{l}\text { Under construction, } \\
\text { Operation in } 2023\end{array}$ & $\begin{array}{l}\text { Under construction, } \\
\text { Operation in } 2023\end{array}$ \\
\hline Reference & {$[268,278]$} & [279] & [253] & [249] & {$[280,281]$} & {$[282]$} & \\
\hline
\end{tabular}


(100-500 $\gamma / \mathrm{s})$, Ballam et al [227] at Stanford Linac Accelerator Center (SLAC) were able to carry out the first physics measurements using a Compton $\gamma$-ray beam to study the photoproduction cross sections at several GeVs with a hydrogen bubble chamber. In 1978 the first Compton gamma-ray source (CGS) facility for nuclear physics research, the Ladon project, was brought to operation in Frascati [228-232]. A higher $\gamma$ flux was produced by colliding the high intensity photon beam inside a laser cavity with the electron beam in the straight section of the $1.5 \mathrm{GeV}$ ADONE storage ring at Frascati National Laboratory. This facility produced a polarized $\gamma$-ray beam with energies up to $80 \mathrm{MeV}$ and an on-target flux of up to $5 \times 10^{5} \gamma / \mathrm{s}$. Following the success of the Ladon facility at Frascati, several more Compton CGS facilities for nuclear physics research were brought to operation around the world starting in the 1980s, including LEGS [233] and HIGS [234] in the US, Graal in France [235], ROKK1/ROKK-2/ROKK-1M in Russia [236-239], and LEPS [240, 241] in Japan. In addition, several other projects successfully produced Compton $\gamma$-ray beams, including FEL-X/AIST [242], UVSOR-FEL/UVSOR-II/UVSOR-III [243-245], NIJI-IV [246, 247], SAGA-LS [248], and NewSUBARU [249] in Japan, and Super-ACO [250, 251] in France. Some specialized CGSs were developed for industrial and national security applications [252-255], such as the MEGaray project [256-258] at Livermore National Lab, US, while advancing certain important areas of accelerator and laser technologies for CGSs [259, 260]. A few published reviews on Compton $\gamma$-ray beams and some of the aforementioned facilities are found in [261-265].

Among these Compton CGS facilities, the High Intensity Gamma-ray Source (HIGS) at Duke University is the first dedicated Compton gamma-ray facility employing as the photon driver a high intra-cavity power free-electron laser (FEL) [266-270]. The HIGS facility is a high-flux, nearly monochromatic, and highly polarized CGS (with both linear and circular polarizations) covering a wide range of energies from about 1 to $100 \mathrm{MeV}$. The HIGS has demonstrated an unprecedented high flux operation with a maximum total flux of about $3 \times 10^{10} \gamma / \mathrm{s}$ around $10 \mathrm{MeV}$, two or three orders of magnitude more flux than what was produced by other CGS facilities ever built and operated. The high flux capability of HIGS is made possible by the full-energy top-off booster injector and recent upgrades to the FEL optical cavity. The wide energy range and high flux performance of the HIGS makes it a unique and superior gamma-ray facility [271-273], ideal for low and medium energy nuclear physics research. It is envisioned extensions that will first increase the energy reach of HIGS up to $130 \mathrm{MeV}$, and then up to $150 \mathrm{MeV}$.

During the last decade or so, while a few CGS facilities (e.g. LEGS and Graal) ceased operation after completing their research missions, other CGS facilities continue to flourish with accelerator and laser system upgrades that improve beam performance and enable new capabilities. In the meantime, a few new CGS facilities are under construction around the world with $\gamma$-ray beam research and user operation expected to start in the next few years. A list of major operational laser Compton CGSs and new development projects is provided in table 10 .

5.1.1. A brief review of Compton scattering process. Compton scattering between an electron and a photon is shown in figure 34. We can list the four momenta of the electron and photon before and after collision as the following,

Using conservation of four-momentum, we can express the energy of the outgoing photon as:

$$
E_{\gamma} \equiv \hbar \omega^{\prime}=\frac{\hbar \omega\left(1-\beta \cos \theta_{\mathrm{i}}\right)}{1-\beta \cos \theta_{\mathrm{f}}+\frac{\hbar \omega}{\mathcal{E}_{\mathrm{e}}}\left(1-\cos \theta_{\mathrm{ph}}\right)},
$$




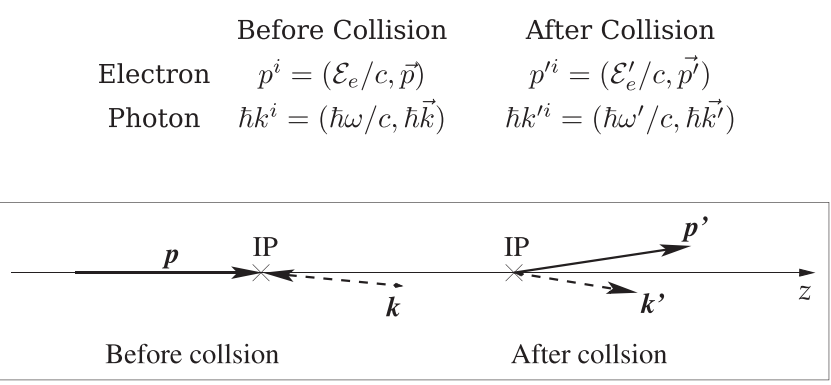

Figure 34. Schematic diagram of Compton scattering of an electron and a photon. IP stands for the interaction point.

where $\hbar$ is the reduced Planck constant, $\hbar \omega$ is the energy of the incoming photon, $E_{\gamma}$ (or $\hbar \omega^{\prime}$ ) is the energy of the outgoing photon, $\theta_{\mathrm{i}}$ and $\theta_{\mathrm{f}}$ are the angles between the momentum of the incoming and outgoing photons and that of the incident electron, $\left(\cos \theta_{\mathrm{i}}=\hat{p} \cdot \hat{k}\right.$ and $\left.\cos \theta_{\mathrm{f}}=\hat{p} \cdot \hat{k^{\prime}}\right)$, and $\theta_{\mathrm{ph}}$ is the angle between the two photons $\left(\cos \theta_{\mathrm{ph}}=\hat{k} \cdot \hat{k}^{\prime}\right)$.

For a collision between relativistic electrons and low energy photons, the energy of the scattered photons is peaked along the direction of the incident electrons. The back-scattered photon has the maximum energy in a head-on collision with $\theta_{\mathrm{i}}=\pi$ and $\theta_{\mathrm{f}}=0$,

$$
\hbar \omega^{\prime}=\frac{\gamma^{2}(1+\beta)^{2}}{1+R_{0}} \hbar \omega
$$

where $R_{0}=2 \gamma^{2}(1+\beta) \hbar \omega / \mathcal{E}_{\mathrm{e}}$ is the recoil factor. When the recoil is small $\left(R_{0} \ll 1\right)$, the maximum scattered photon energy is approximately, $E_{\gamma, \max } \approx \gamma^{2}(1+\beta)^{2} \hbar \omega \approx 4 \gamma^{2} \hbar \omega$, where the second approximation holds for ultra-relativistic electrons. The energy boost factor, $4 \gamma^{2}$, is responsible for the relativistic Doppler effect (or relativistic blue-shift) to transform an ordinary infrared or visible light photon into a high energy $\mathrm{x}$-ray or $\gamma$-ray photon.

At the zero-recoil limit, the total cross section is rather small $\sigma \approx \frac{8 \pi r_{\mathrm{e}}^{2}}{3}=6.652 \times 10^{-29} \mathrm{~m}^{2}$ (i.e., the Thomson cross section). For the collision of a relativistic electron beam and a lowenergy photon beam, scattered photons with the highest energy are concentrated around the direction of the incident electron beam. This kinematic feature enables the formation of a nearly monochromatic Compton photon beam by using a simple collimation technique.

\subsection{Technology for next-generation Compton gamma-ray sources}

Limited by the available flux and spectral flux of present and future Compton CGSs, and complex event detection and beam diagnostic techniques used for measurements, the exploration of many important nuclear physics phenomena with small cross sections can be best realized using a $\gamma$-ray beam with a high repetition rate. Compared with such a CW beam, without being able to explore the multi-photon effect due to the intensity limitation and lack of coherence, a lowrepetition-rate pulsed Compton $\gamma$-ray beam does not have practical advantages in most cases [expect for certain experiments which can exploit extremely fast (short) pulses]. In developing a CGS with a high average flux or spectral flux, the preferred technology choice demands a combination of an electron accelerator and a laser system with their respective high beam repetition rate matched. For the next-generation Compton CGSs, we focus on the following optimum technology choices:

- Electron accelerators: a storage ring (a well-established technology) or a super-conducting linac (a newer technology) 
- Photon beam systems: a high-finesse resonant optical cavity

- Interaction point (IP) configuration: electron-photon collision inside the laser cavity.

Using an external laser beam in a simple optical setup for collision has some attractive features, especially when combining a low repetition rate, high peak power laser with a warm electron linac. However, this scheme is not cost-effective for generating $\mathrm{CW} \gamma$-ray beams due to its inefficiency in utilizing the photon beam in a single pass. The use of an optical resonator for in-cavity collision allows the electron beam to interact with the accumulated laser power repeatedly, thereby reusing the photon beam in a large number of passes. The in-cavity collision scheme can be arranged in either a head-on configuration or a cross-angle configuration. While the head-on configuration has the advantage of a higher luminosity, it is more limiting as it requires a mechanism to guide the electron beam into and out of the optical cavity. Meeting this requirement would limit the geometric design of the resonator and complicate the magnetic optics design around the IP. On the other hand, the cross-angle scheme is compact and can be arranged to use a relatively short resonator. Because of these and other benefits and shortcomings, both head-on and crossed-angle collision configurations should be considered for the development of the next-generation Compton CGSs.

In the following sections, a brief overview of the electron beam and photon beam technologies is given. To mitigate many potential risks, the focus will be on conventional, wellestablished, mature or fast-maturing technologies for the next-generation Compton CGSs which require an unprecedented level of flux, energy resolution, stability, reliability, and precise control and manipulation. Before going into these topics, a brief digression into other technological developments and advancements relevant to the Compton CGSs is in order.

5.2.1. Related technology development. Technological advancements relevant to the Compton CGSs have been reviewed and summarized in the 2010 US DOE Basic Energy Sciences sponsored workshop on Compact Light Sources [279]. This workshop examined the readiness of state of the art technology for compact light sources (including so-called inverse Compton scattering sources') including assessing cost-effectiveness, user access, availability, and reliability. The report also compared the performance of compact light sources to the thirdgeneration storage rings and FELs. While the details can be found in the report, we will briefly describe and give updates to the two areas of technological development that can impact the development of Compton CGSs in the future.

5.2.1.1. Compton $x$-ray sources. Since mid 1990s, x-rays have been generated by means of Compton scattering (also often referred to as 'Thomson scattering' due to negligible electron recoil) using low energy electron beams. The relatively low cost and/or good availability of lower energy conventional accelerators have allowed the exploration of laser Compton scattering with a variety of combined accelerator and laser technologies:

- Combining a warm s-band linac and a pulsed laser: a $30 \mathrm{keV}$, femtosecond x-ray beam was generated with a $90^{\circ}$ cross-angle at Berkeley National Laboratory [280]; a very high peak flux X-ray beam $\left(2 \times 10^{9}\right.$ up to $10^{10} \mathrm{ph} /$ pulse $)$ was produced at Vanderbilt University [281]; a $52 \mathrm{keV}$ x-ray beam was generated at Tsinghua University, China with controllable polarization [282, 283]; and an $11 \mathrm{keV}$ x-ray beam was produced at CEA, France, by utilizing a ring-down laser cavity with a folded laser path [284];

- With a linac FEL: tunable 7 to $12 \mathrm{keV}$ x-rays were demonstrated at CLIO, France, using an infrared linac FEL powered by the same electron beam [285];

- With a superconducting linac: a tunable 3.5 to $18 \mathrm{keV}$, high average flux (few $10^{9} \mathrm{ph} / \mathrm{s}$ ) $\mathrm{X}$-ray beam was generated using a kW-class infrared FEL at Jefferson Lab [286]; $29 \mathrm{keV}$, 
millisecond long X-ray pulses (macropulses) were demonstrated with a four-mirror optical cavity at KEK, Japan [287]; the generation of $7 \mathrm{keV}$ x-rays with a CW electron beam was demonstrated with an energy recovery linac (ERL) and a four-mirror cavity [288];

- Combining a storage ring and a high-finesse Fabry-Perot (FP) cavity: likely the highest average flux X-ray beam was delivered by a compact X-ray source system manufactured by Lyncean Technologies Inc. using a dedicated storage ring and a high power, four-mirror FP cavity powered by an external Nd:YAG laser [289]. Using the same technology combinations, an even higher flux Compton X-ray source, THOMX, is now under construction in Orsay, France [290, 291].

The above brief survey of this necessarily incomplete list of Compton x-ray source projects worldwide in the recent two decades has also confirmed that the optimum technological combination to generate a high average photon flux is to use a high repetition rate electron beam to collide with a frequency matched laser beam inside an optical resonator.

5.2.1.2. Laser-plasma accelerator based Compton sources. Compton photon sources continue to be a vibrant research area. In additional to the aforementioned sources driven by conventional accelerators, new Compton sources based on laser-plasma accelerators [292, 293] have been actively developed around the world in the recent decade [294-302]. A few published reviews describing laser-plasma acceleration, ultra-fast Compton photon generation, and applications of such light sources can be found in [303-305]. In these relatively compact and all-optical Compton sources, the ultra-short, high-peak power laser plays two essential roles: (1) generating and accelerating electrons, and (2) as an intense photon drive to collide with the electrons. With adequate peak laser intensity the laser-plasma Compton sources are wellsuited to explore a new operational regime of nonlinear Thomson/Compton scattering in which multiple photons collide with a single electron to generate one Compton photon with energy much higher than in the linear Compton scattering. Various experiments using different lasers and targets (solids, gases) have proved the feasibility of this method and there is a long term path to high beam quality. However, a specific set of beam quality at this time, including the beam flux, energy spread, spectral flux, spectral purity, pointing stability, etc is less desirable compared with the Compton sources driven by conventional accelerators. On the other hand, certain unique characteristics of a laser-plasma Compton source (such as ultrafast pulses, compactness, etc) have yet to be adequately exploited for basic and applied science research [306, 307]. While providing promise for the future, at the present time, the laser-plasma Compton systems are still at an experimental exploration stage, therefore, not ready to implement for a user facility.

\subsubsection{New developments.}

5.2.1.3.1. ELI-NP A major CGS under construction is the Extreme Light InfrastructureNuclear Physics (ELI-NP), a European Center of Excellence for scientific research in laser and gamma radiation. The facility is located in the town of Magurele near Bucharest, Romania. ELI-NP's new CGS, the variable energy gamma (VEGA) system is a dedicated system for delivering $\gamma$-ray beams to users. The construction of the VEGA system was recently awarded to Lyncean Technologies Inc. and its delivery, installation and acceptance are scheduled to be completed in early 2023.

The VEGA system will deliver $\gamma$-rays with energy continuously variable from 1 to 19.5 MeV, covering the energy range relevant for low energy nuclear physics and astrophysics studies, as well as applied research in material sciences, management of nuclear materials, and life sciences. The beams will be quasi-monochromatic with a relative bandwidth better than $0.5 \%$ (FWHM), high intensity with a spectral density higher than $5 \times 10^{3} \mathrm{\gamma} / \mathrm{eV} \mathrm{s}$, and a high 
degree of linear polarization at more than $95 \%$. With these parameters, the VEGA system will be the most advanced CGS in the world having about one order of magnitude higher $\gamma$-ray flux and at least a factor of two smaller relative bandwidth than the current state-of-the-art [274]. The VEGA system is based on the use of a storage ring and a high-finesse FP cavity. The parameters of the electron beam and the interaction laser are optimized at the IP in a way to provide $\gamma$-rays with the features discussed above. The electron beam system will operate in the range of 234-742 MeV. For a given interaction laser wavelength, this electron energy range allows at least a factor of ten in $\gamma$-ray energy continuous tunability. Two separate optical cavity laser systems, one at $\sim 1 \mu \mathrm{m}$ ('IR') and the other at $\sim 0.5 \mu \mathrm{m}$ ('green') wavelengths, will be provided to cover the $\gamma$-ray energy range from 1 to $10 \mathrm{MeV}$, or 2 to $19.5 \mathrm{MeV}$, respectively. The laser systems use a passive, high-finesse optical cavity to resonantly build-up the pulsed laser power. The optical cavity provides gains of 5000-10000 in laser power, which reduces the complexity of the interaction laser drive system. The main parameters for the VEGA system are summarized in table 10 .

5.2.1.3.2. Gamma Factory at CERN A new initiative at CERN, the Gamma Factory (GF) [308], is under rapid development in the last two years. The GF project is aimed at creating, storing, and exploiting new types of relativistic atomic beams. These atomic beams of ions with all but a few electrons stripped can be stored in the Super Proton Synchrotron (SPS) or the Large Hadron Collider (LHC) storage rings at very high energies $(30<\gamma<3000$ ), at high bunch intensities $\left(10^{8}<N_{\text {bunch }}<10^{9}\right.$ ), and at high bunch repetition rate (up to $20 \mathrm{MHz}$ ). With the GF approach, a resonant atomic transition of a highly-charged relativistic ion excited by laser light results in a spontaneously emitted photon. Due to the relativistic Doppler effect, the photons emitted in the direction of the ions can reach very high energies, ranging from 1 to $400 \mathrm{MeV}$ for the atomic beams stored in the LHC rings. Because of a huge resonant photon absorption cross section compared to that of photon scattering with a point-like electron (a factor up to $10^{9}$ ), the intensity of an atomic-beam-driven light source is expected to be several orders of magnitude higher than what is possible with Compton CGSs driven by an electron beam, reaching a total $\gamma$-ray flux up to $10^{17} \gamma / \mathrm{s}$ [309].

To prove experimentally the concepts underlying the GF proposal, feasibility tests have been and will continue to be performed at the SPS and at the LHC. Since 2017 the experimental beam tests have started with partially stripped ${ }^{129} \mathrm{Xe}^{39+}$ beams, followed by dedicated SPS runs with ${ }^{208} \mathrm{~Pb}^{54+},{ }^{208} \mathrm{~Pb}^{80+},{ }^{208} \mathrm{~Pb}^{81+}$ beams. The ${ }^{208} \mathrm{~Pb}^{81+}$ beam was injected for the first time into the LHC and ramped to a proton equivalent energy of $6.5 \mathrm{TeV}$ in 2018 [310]. The majority of the operation aspects for such beams have been successfully tested. An important specific achievement was to demonstrate that bunches of $10^{8}$ hydrogen-like lead atoms per bunch can be efficiently produced and maintained at the LHC top energy with the lifetime and intensity fulfilling the GF requirements. The pivotal concept of the GF initiative-that relativistic atomic beams can be produced, accelerated and stored in the existing CERN SPS and LHC rings-has therefore already been experimentally proven.

The SPS and LHC beam tests will be followed by the GF 'proof-of-principle' SPS experiment [311]. This experiment will study collisions of a laser beam with the ${ }^{208} \mathrm{~Pb}^{79+}$ beam in a specially designed collision point in the SPS tunnel. Its results will provide a decisive proof and an experimental evaluation of the achievable intensities of the atomic-beam-based CGS.

The success of CERN's GF initiative can have a profound impact to the worldwide effort in developing next-generation Compton CGSs. 


\subsubsection{Electron accelerators.}

5.2.2.1. Electron storage rings. The development and operation of a number of storage ring based third-generation light sources since the 1980s [312-316] has significantly advanced the science and technology of the electron storage ring. In particular, advancements have been made in the following critical areas:

- Understanding and managing charged particle nonlinear dynamics to realize strong focusing magnetic optics with very small transverse beam emittance at nanometer-radians;

- Mitigating and controlling a variety of collective effects in the storage ring to enable high current operation; and

- Improving flexibility, stability and availability of storage ring light sources using advanced beam diagnostics and accelerator control systems.

In the area of nonlinear dynamics, the more recent development of storage ring based, diffraction limited light sources has further advanced the control and compensation of nonlinear effects in the storage ring lattice using multi-bend achromats (MBA), achieving an unprecedented level of tens of picometer-radian beam emittance [317-320]. The development of MBA based diffraction limited light sources has also advanced the design and manufacture of high-field and high-quality magnets and small vacuum chamber systems. Many effective technologies have been developed to mitigate several important collective effects, such as microwave instability, intra-beam scattering, and coupled-bunch instabilities using uniform and smooth vacuum chambers, harmonic rf cavities, and bunch-by-bunch feedback systems [321-325]. A variety of beam diagnostic and control techniques, such as slow/fast orbit feedback, beam based lattice calibration, fast focusing compensation, etc, have greatly improved the storage ring operation stability and consistency [326-336]. Furthermore, the development of sophisticated slow accelerator controls has expanded the storage ring capabilities by allowing a rapid change among a variety of operation modes with different bunch patterns, beam currents, and beam energies [337-343].

The storage ring is a mature accelerator technology, well-suited as the electron beam driver for the next-generation Compton CGS. In fact, the next-generation CGS requires only a modest beam emittance (a few to tens of nm rad), a reasonably high current (an average current from $100 \mathrm{~s} \mathrm{~mA}$ to $1 \mathrm{~A}$ ), and a level of beam stability achievable using relatively long bunches (tens to hundreds of picoseconds) (see the example CGSs below). Without a need to push the limits of the accelerator technology, a storage ring based CGS can be developed and constructed with a modest cost, a well-defined schedule, and a manageable level of risk. This technology is also being constantly improved and optimized with several existing Compton CGSs (see table 10). Overall, the electron storage ring is a highly recommended accelerator technology for the next-generation CGS.

5.2.2.2. Superconducting linacs. Superconducting linacs are the heart of ERLs. ERLs can provide high brightness electron beams at high average current (tens of milliamperes). This allows them to be used in applications where storage rings normally operate. They are well suited to CGS applications due to the ability to tolerate relatively high beam losses caused by the energy loss of electrons that emit high energy $\gamma$-rays.

Superconducting linacs continue to improve in terms of gradient, efficiency, and current. The LCLS II program at SLAC is producing $12 \mathrm{~m}$-long cryomodules with over $128 \mathrm{MV}$ of CW accelerating voltage and the LCLS II HE program will demand cryomodules with over $158 \mathrm{MV}$ CW accelerating voltage [344]. In both cases the cavity unloaded quality factor $Q_{0}$ must be greater than $2.7 \times 10^{10}$ at $2.0 \mathrm{~K}$. This allows high gradients without requiring excessive refrigeration. Source technology has also advanced both at Cornell and Brookhaven National 
Lab. The LEReC program at Brookhaven has recently used a $30 \mathrm{~mA}$ electron beam with excellent beam quality to cool ions in the RHIC accelerator [345]. The beam was delivered 24/7 for a user program, thus showing sufficient reliability for a gamma source as well. The Compact ERL (cERL) at KEK in Tsukuba Japan has recirculated $1 \mathrm{~mA}$ of beam current in an x-ray Compton backscattering experiment [346]. Ultimately one would like to use SRF cavities at 4.5 K rather than the less efficient $2 \mathrm{~K}$ operation. This can be achieved via the use of niobiumtin coated SRF cavities [347]. Recent experiments at Jefferson Lab have demonstrated over $14 \mathrm{MV} \mathrm{m}^{-1}$ in a five-cell $1.497 \mathrm{GHz}$ cavity. The cavity $Q_{0}$ was $2 \times 10^{10}$ at $4.5 \mathrm{~K}$. This effort aims at producing cavities with a $Q_{0}$ of $10^{11}$ at $20 \mathrm{MV} \mathrm{m}^{-1}$ at $4.5 \mathrm{~K}$.

Another area of advancement is the idea of using multiple passes through the accelerating cavities with energy recovery. For systems with a total energy less than $1 \mathrm{GeV}$ this can produce a high current, compact accelerator. Researchers at the Daresbury Laboratory have produced a design for a $1 \mathrm{GeV}$ recirculating linac called DIANA that would require only two cryomodules of $165 \mathrm{MV}$ acceleration apiece. This could be a very potent CGS [348]. Cornell is using a fourpass configuration to get to $150 \mathrm{MeV}$ with one cryomodule. The design current is $30 \mathrm{~mA}$. The machine is installed and being commissioned [349].

5.2.3. Photon beam systems. Various optical systems have been used to increase the available laser beam intensity in Compton scattering experiments. Firstly, 'recirculator' systems [350] have demonstrated effective laser beam energy gains with an enhancement factor between 8 [284] and 20 [351] (an enhancement factor of about 30 is foreseen in reference [352]). Secondly, a FP cavity (optical resonator), a well-known optical device [353-355], can provide gain in excess of $10^{3}$. We shall concentrate here on 'external cavities' which are filled by external laser beams (i.e., not FELs). These devices can also act as circular [356] or linear polarization filters $[357,358]$ by geometrical or coating designs. Optical path length inside optical resonators ranges between micrometers up to $30 \mathrm{~m}$ [359]. This latter number sets a reasonable minimum laser pulse repetition rate at about $10 \mathrm{MHz}$, making FP cavity especially useful for $\mathrm{CW}$ electron beams (i.e., in a storage ring or superconducting linac). Nevertheless, it has recently been demonstrated at KEK [360] that FP cavity operated in a 'burst mode' [360] can also be used advantageously with a warm linac. In these devices, laser oscillators must be tightly locked to the resonator round-trip length. Highly efficient feedback methods are indeed well known [361] and have been extended to mode lock regime [362-365].

FP cavities using either two mirrors or four mirrors are of particular interest to achieve high gains. Two-mirror high-finesse $(F \sim 30000)$ cavities operating with CW Nd:YAG oscillators have been used successfully for Compton polarimeters [366, 367] and Compton laser wire [368]. Moderate average power (few kW) has been routinely obtained with these cavities. However, two mirror cavities cannot provide stability and very strong focusing simultaneously. In addition, the cavity length tuning required for timing synchronization with the electron beam is not independent of the beam focus tuning. A good alternative is four-mirror cavities. They provide at the same time stability, strong focusing and an independent tuning of the cavity length and beam focusing. A main drawback with a four-mirror cavity is that the cavity modes are elliptical.

In the pulsed regime, the following state-of-the-art FP cavity performance has been obtained with table-top systems:

- High average power: about $700 \mathrm{~kW}$ (10 ps laser pulses) with a Yb-doped oscillator and a laser amplifier delivering more than $400 \mathrm{~W}$ [369].

- High finesse: a finesse of approximately 30000 (power enhancement factor of 10000 ) using a low power Ti:sapphire (3 ps laser pulses) oscillator [370]. 
Combining high average power and high finesse is presently under investigation in the context of compact Compton x-ray sources. Various optical cavities have already been implemented with an electron storage ring, a linac and ERL at KEK. For instance:

- Non-planar four-mirror cavity (178.5 MHz) filled by a 10-20 W Yb-doped, picosecond oscillator/fiber-amplifier system [371]. Up to $50 \mathrm{~kW}$ stored power was obtained routinely [372].

- Planar four-mirror cavities (357 MHz and $162.5 \mathrm{MHz}$ ) filled by a Nd-doped, picosecond oscillator/burst-amplifier systems [373, 374].

A four-mirror cavity with $70 \mathrm{~kW}$ stored power (with Nd-doped $65 \mathrm{MHz}$ oscillator) is also routinely operated in a commercial Compact x-ray source [289].

From the above results, up to $\sim 100 \mathrm{~kW}$ average power in picosecond regime can be obtained by careful choice of the mirror substrates/coatings [369] and careful mechanical design. Low noise oscillators recently available enable the use of a high-finesse cavity in order to reduce the cost of high average power laser amplifiers. Eventually, to avoid mirror damage and to optimize the laser beam shape at the Compton IP, various cavity geometry and boundary shapes have been proposed [375-379].

Compared to a compact, high-power FP cavity driven by a multiple MHz external laser, a larger-scale and more complex FEL oscillator remains an attractive option as the photon beam drive for a high energy CGS because of its demonstrated extraordinary versatility in many areas. The oscillator FEL is well-known for its wavelength flexibility (e.g., the Duke storage ring FEL can be operated from $2.1 \mu \mathrm{m}$ to $188 \mathrm{~nm}$ ). More recently, the storage ring FEL has been developed to demonstrate several new capabilities which can have an important impact for a CGS, including simultaneous two-color lasing $[380,381]$ and the use of an 'optics-free' method to rapidly control and manipulate the laser beam (and $\gamma$-ray beam) polarization with an unprecedented level of precision [269, 382].

\subsection{Next generation Compton gamma-ray sources: examples and capabilities}

In this section, we describe several possible ways to develop next-generation CGSs based upon different technology choices. These example CGSs take advantage of the most recent advancements in laser and accelerator technologies to achieve the key beam parameters required by science programs. They are practical with manageable levels of risk-without pushing multiple limits of laser and accelerator science and technology, and these CGSs can be designed and constructed in the next five to ten years. These sources are expected to be reliable, especially for those adopting mature and established technological solutions; and some are also well-suited for multi-user operation. Depending on the $\gamma$-ray beam energy range and technology choices, the cost for such a CGS varies significantly.

Beam performance requirements are specified by the research opportunities described in the previous science sections of this report. We envision two types of facilities needed to satisfy the user needs in the low-energy and medium-energy ranges. Beam requirements are organized using a few key parameters, such as the beam energy, intensity, energy resolution, polarization, and repetition rate in tables 11 and 12 .

Using two main $\gamma$-ray beam parameters, the energy range and intensity, a comparison of the example CGSs considered for the next generation CGS are provided in table 13. In this table, comments on the risk level for each source are provided and several main challenges are identified. For other beam parameters, the relevant details are provided in the individual section for each source. 
Table 11. Preliminary medium-energy $\gamma$-ray beam requirements for low energy QCD research. Note that the energy resolution requirement can be relaxed to 3\% (FWHM) for higher energies $(>150 \mathrm{MeV})$.

\begin{tabular}{lccccc}
\hline $\begin{array}{l}\text { Repetition rate } \\
(\mathrm{MHz})\end{array}$ & $\begin{array}{c}E_{\gamma} \\
(\mathrm{MeV})\end{array}$ & $\begin{array}{c}\text { Flux on target } \\
(\gamma / \mathrm{s})\end{array}$ & $\begin{array}{c}\text { Energy resolution } \\
(\text { relative, FWHM })\end{array}$ & $\begin{array}{c}\text { Total flux Est. } \\
(\gamma / \mathrm{s})\end{array}$ & Polarization \\
\hline 10 & $60-350$ & $3 \times 10^{8}$ & $1.5 \%-2 \%$ & $1-1.5 \times 10^{10}$ & $\begin{array}{c}\text { Cir., flip; } 10 \mathrm{~Hz} \\
\text { Lin., flip; } 10 \mathrm{~Hz}\end{array}$ \\
$20-30$ & $100-300$ & $1 \times 10^{9}$ & $2 \%$ & $3-4 \times 10^{10}$ & Cir; \\
Up to 200 & $100-300$ & $1 \times 10^{9}$ & $2 \%$ & $3-4 \times 10^{10}$ & Cir \\
\hline
\end{tabular}

Table 12. Preliminary low-energy $\gamma$-ray beam requirements for nuclear astrophysics and nuclear structure research. The nuclear structure research requires $\gamma$-ray beams with a repetition rate not exceeding $100 \mathrm{MHz}$.

\begin{tabular}{lcccrc}
\hline Research area & $E_{\gamma}(\mathrm{MeV})$ & $\begin{array}{c}\text { Flux on } \\
\text { target }(\gamma / \mathrm{s})\end{array}$ & $\begin{array}{c}\text { Energy resolution } \\
(\text { relative, FWHM })\end{array}$ & $\begin{array}{r}\text { Total flux Est. } \\
(\gamma / \mathrm{s})\end{array}$ & Polarization \\
\hline Nuclear astrophysics & $2-5$ & $1 \times 10^{11}$ & $1 \%$ & $0.8-1 \times 10^{13}$ & Any \\
& $5-10$ & $1 \times 10^{10}$ & $1 \%$ & $0.8-1 \times 10^{12}$ & Any \\
Nuclear structure HPV & $10-20$ & $5 \times 10^{9}$ & $1 \%-2 \%$ & $2-5 \times 10^{11}$ & Any \\
Few-nucleon nuclei & $2-5$ & $1 \times 10^{10}$ & $0.5 \%-1 \%$ & $4-8 \times 10^{10}$ & Lin. or Cir. \\
Parity doublets in nuclei & $2-10$ & $1 \times 10^{9}$ & $0.5 \%-1 \%$ & $1 \times 10^{10}$ & Cir. \\
\hline
\end{tabular}

5.3.1. Storage ring based medium energy CGS. The required $\gamma$-ray beam performance at higher energies (table 11) can be realized with a storage ring based Compton CGS by colliding a $\mathrm{GeV}$ electron beam with a high-repetition laser beam (tens of $\mathrm{MHz}$ ) inside a FP cavity with modest intracavity power. One possible design of such storage rings is shown in figure 35 with related machine parameters listed. This $200 \mathrm{~m}$-long racetrack storage ring is comprised of two multi-bend achromatic arcs with a small emittance and a reasonably large dynamic aperture for the electron beam injection using conventional kickers. The performance projection of such a Compton CGS is provided below for a cross-angle collision configuration $\left(\theta=6^{\circ}\right)$.

The storage ring should be developed to operate in a wide range of energies using specialized equipment with a large dynamic range of stability and reliability, including power supplies, magnets, kickers, beam diagnostics, etc [264, 383]. The flux performance of this medium energy CGS will be limited by the electron loss rate. To produce a medium energy $\gamma$ ray beam, a significant amount of energy is transferred from an electron to the photon, causing the electron to be lost outside the energy acceptance of the storage ring. In this electron-loss mode, the $\gamma$-ray flux will be limited by the electron injection rate into the storage ring. Instead of pushing the emittance limit, this storage ring should be optimized to store substantial beam currents and to allow flexible injection.

Energy range: $\gamma$ rays in a wide energy range can be produced from $25 \mathrm{MeV}$ to about $400 \mathrm{MeV}$ (see the table in figure 36). This can be done by operating the storage ring at energies between 1.2 and $3.5 \mathrm{GeV}$, and by using one or more FP cavities at a few selected wavelengths between $1550 \mathrm{~nm}$ and $517 \mathrm{~nm}$.

Flux performance: the flux requirements in table 11 can be realized readily. A $\gamma$-ray beam with a total flux of about $3.4 \times 10^{11} \gamma / \mathrm{s}$ (in the $4 \pi$ solid angle) can be produced in a wide energy range. For example, a $350 \mathrm{MeV} \gamma$-ray beam of this intensity with a $95.4 \mathrm{MHz}$ repetition rate can be produced using a $500 \mathrm{~mA}$ electron beam $(3.27 \mathrm{GeV})$ and a modest $20 \mathrm{~kW}$ laser power 
Table 13. Comparison of several example CGSs based upon different technology choices, in terms of their energy range, beam intensity, and risk levels. The projected total flux is made under the assumption of a high repetition rate beam production, and the flux on target is estimated for a properly collimated beam with a relative energy resolution of 2\% (FWHM) (about 3\% of the total flux). Note that the head-on collision is much more efficient in producing high $\gamma$-ray beam intensity, however, it is very challenging to simultaneously realize this collision geometry and a very small laser beam size at the collision point $\left(\sigma_{x, y}<30 \mu \mathrm{m}\right)$.

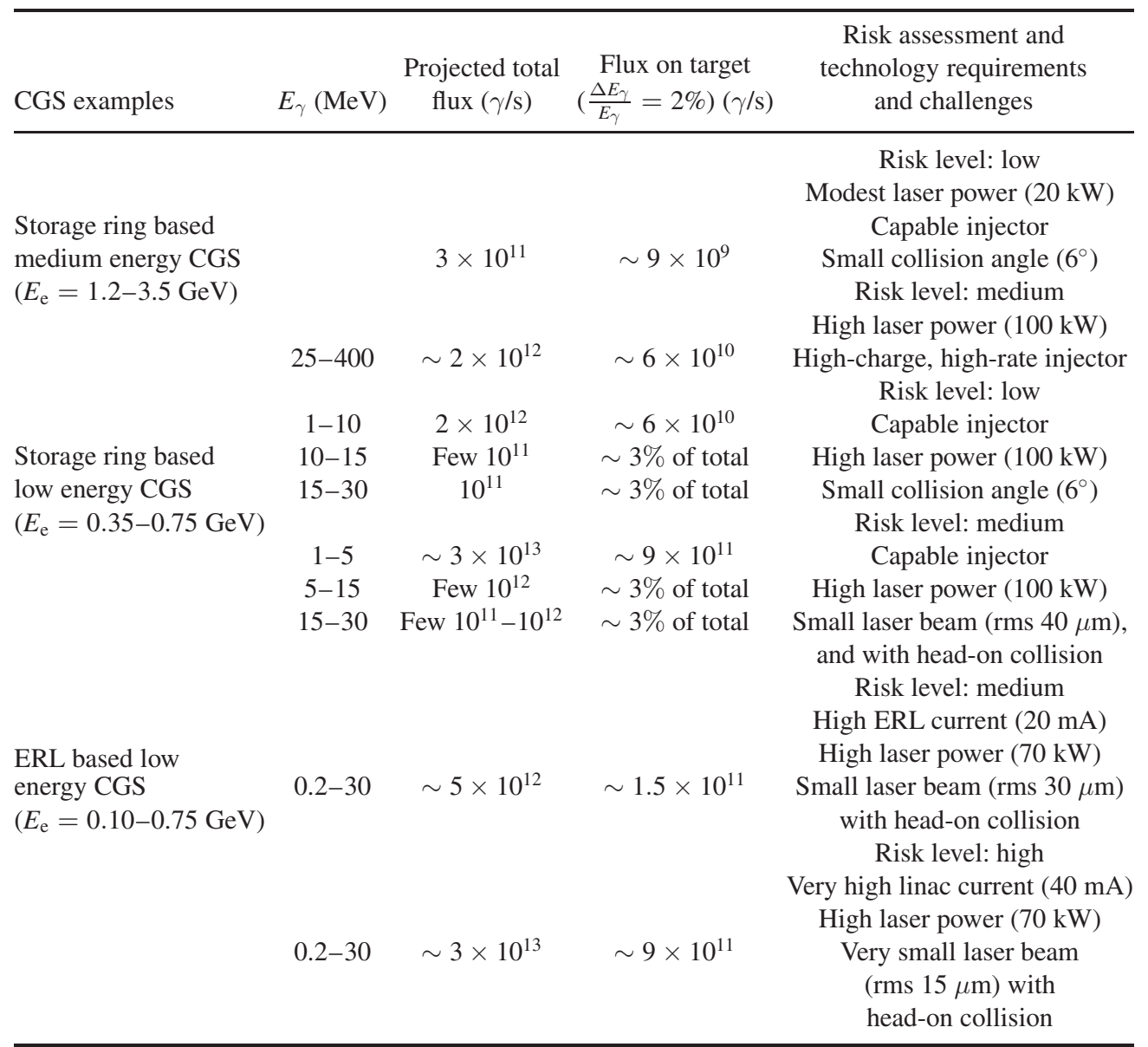

inside a $517 \mathrm{~nm}$ FP cavity (see the detailed beam parameters in the table in figure 36 ). The corresponding collimated flux for a beam with an FWHM energy resolution of $2 \%$ is about $9 \times 10^{9} \mathrm{\gamma} / \mathrm{s}$. For those experiments requiring a lower repetition rate, 6 out of $64 \mathrm{rf}$ buckets can be filled with the same bunch charge to produce a roughly $9.5 \mathrm{MHz}$ beam with a collimated flux of about $8 \times 10^{8} \mathrm{\gamma} / \mathrm{s}$, and higher flux is possible by increasing the bunch charge. Furthermore, by increasing the intracavity laser power to $100 \mathrm{~kW}$ to match the capability of a high charge, high repetition rate booster injector $(32 \mathrm{nC} /$ cycle, $10 \mathrm{~Hz}$ ), the total $\gamma$-ray flux can be increased to about $2 \times 10^{12} \gamma / \mathrm{s}$. Ultimately, the $\gamma$-ray flux for this medium energy CGS will be limited by the electron injection rate, not by the laser power in the FP cavity or the stored beam current in the storage ring. 


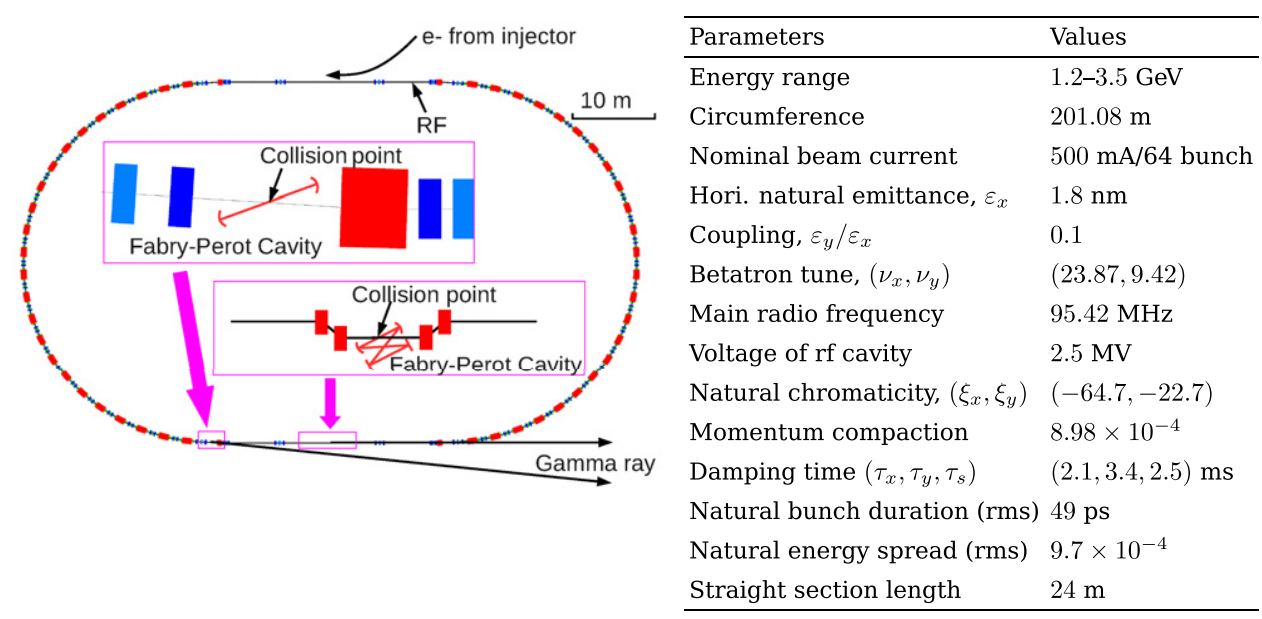

Figure 35. (Left) Layout and parameters of a medium energy electron storage ring $(1.2-3.5 \mathrm{GeV})$. The insets show two possible collision schemes using a two-mirror and a four-mirror FP cavity, respectively (the laser cavity length is not to scale). (Right) Main parameters for the storage ring, where all energy-dependent parameters are shown for $3.5 \mathrm{GeV}$ operation.

Electron beam: to achieve a high average current, the electron beam will be stored in multiple bunches. The bunch pattern should be selectable as demanded for specific user experiments for either high flux operation at a high repetition rate, or a modest flux operation at a lower rate.

Laser beam: the FP cavity will be powered by a commercial laser (fiber or solid state) operated at tens to hundreds of $\mathrm{MHz}$ repetition rate with a picosecond pulse duration. The commonly available wavelengths are around 1064 and $1550 \mathrm{~nm}$; their 2nd and/or 3rd harmonics can also be used with a lower power.

FP cavity and collision scheme: for this medium energy CGS, the required intracavity laser power is rather modest, reducing the need to push the FP cavity power limit. Both head-on collision and small cross-angle collision can be arranged using either a two-mirror or four-mirror cavity. The entire $\gamma$-ray energy range can be covered using one FP cavity specially designed to build up the laser power at both the fundamental wavelength at $1550 \mathrm{~nm}$ and its third harmonic at $517 \mathrm{~nm}$ (see the table in figure 36). To cover a wide intermediate energy range (from 30 to $200 \mathrm{MeV}$ ), a $1064 \mathrm{~nm}$ FP cavity is well suited and highly practical because the FP cavity technology at $1064 \mathrm{~nm}$ is well established and more mature than that for $1550 \mathrm{~nm}$.

Energy resolution: the required $\gamma$-ray beam energy resolution (1.5\%-2\%, FWHM) can be met by using a narrow-band laser beam, and an electron beam with a small energy spread and reasonably small transverse emittance $[384,385]$. The electron beam performance is readily achievable using the state-of-the-art storage ring magnetic optics design, taking advantage of recent advances in developing low-emittance storage ring based synchrotron radiation sources [386, 387].

Beam polarization: a well-collimated Compton $\gamma$-ray beam driven by a polarized laser beam can have a very high degree of polarization, either linear or circular. The relatively slow polarization manipulation (up to $10 \mathrm{~Hz}$ ), switching between two polarization states (the horizontal and vertical, or left and right circular), can be realized using conventional polarizing optics (including Pockels cells) outside the FP cavity where the laser power is much lower. 


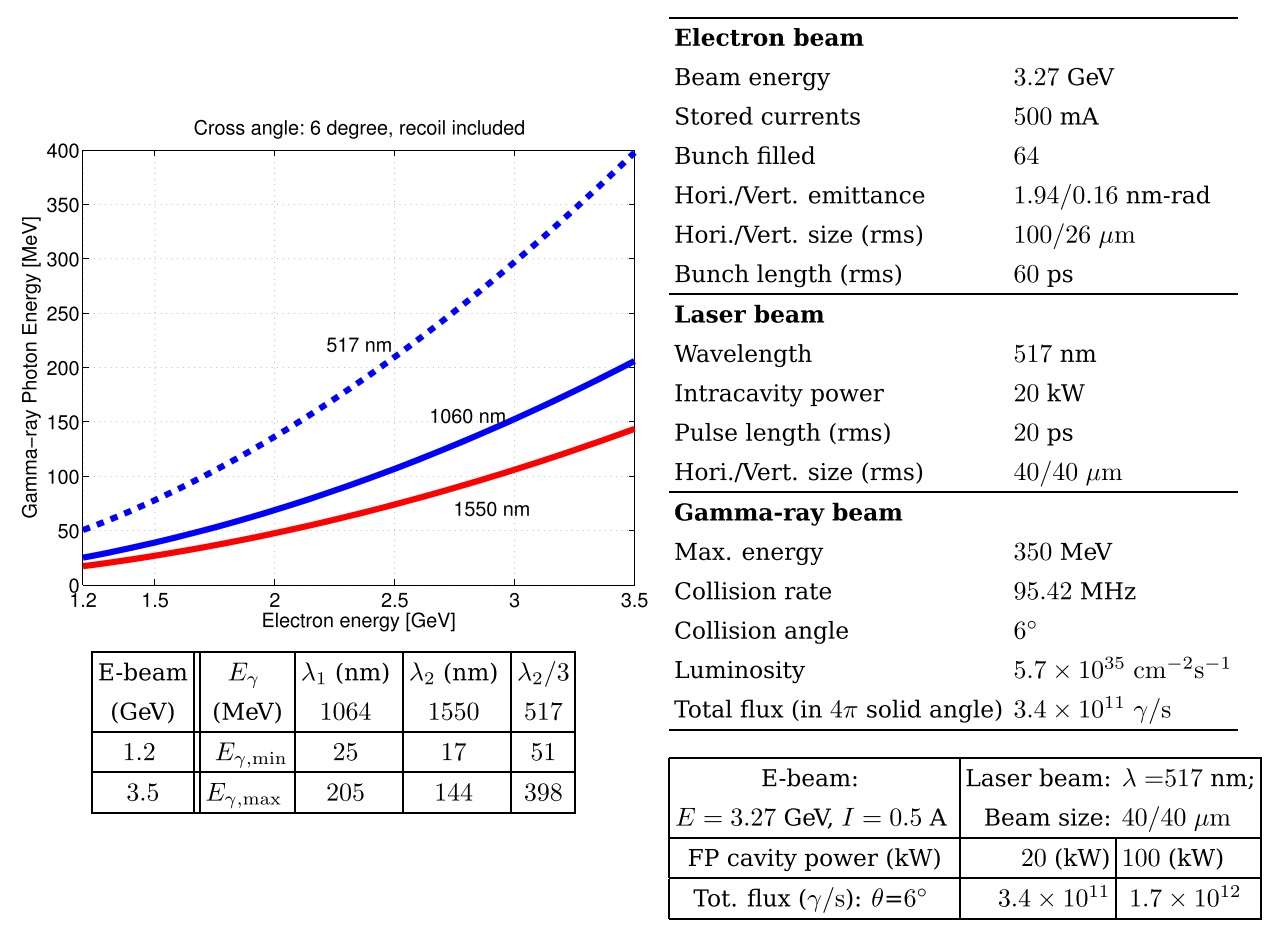

Figure 36. (Left) Energy of the $\gamma$-ray beam as a function of electron beam energy $(1.2-3.5 \mathrm{MeV})$ for lasers at $1064 \mathrm{~nm}, 1550 \mathrm{~nm}$ and its third harmonic at $517 \mathrm{~nm}$ with a $6^{\circ}$ collision angle. (Upper-right) A table listing the main parameters for producing a $350 \mathrm{MeV} \gamma$-ray beam using a $3.27 \mathrm{GeV}$ electron beam and a $517 \mathrm{~nm}$ laser beam. (Lower-right) A table showing the total flux for two levels of intracavity laser powers.

Production modes: the medium energy CGS is likely to be operated as a single- or fewuser facility. Several Compton sources can be installed in one or more straight sections as shown in figure 37. When operated simultaneously, the sum of $\gamma$-ray flux from all sources will be limited by the electron injection rate. For each CGS, a dedicated user target room with proper shielding and a beam dump should be constructed. Multiple target rooms are desirable as complex medium energy experiments will need a dedicated staging area to develop, test, and integrate experimental equipment while the other target room(s) is/are being used for production data-taking.

Several challenges and important issues for this medium energy source are summarized here:

- Electron injection rate: the cost-effective choice for the injection is a full-energy, top-off booster injector. The booster injector needs to be designed to achieve high reliability, a fast injection cycle (up to $10 \mathrm{~Hz}$ ), and a large average injection rate (few $10^{11}$ to few $10^{12} \mathrm{e}^{-} / \mathrm{s}$ ).

- Dual wavelength FP cavity: a dual wavelength FP cavity system will need to be developed to operate at both $1550 \mathrm{~nm}$ and $517 \mathrm{~nm}$ (the $3 \mathrm{rd}$ harmonic). The cavity mirrors should have very high reflectivity at both wavelengths. A new type of optical bench with additional third-harmonic generation optics, two sets of laser optics, feedbacks, and diagnostics for both wavelengths should be developed and then integrated with a shared FP cavity.

- Low beam background: to reduce the high energy radiation background, a short interaction region can be arranged to fully separate the $\gamma$-ray beam from the residual gas 


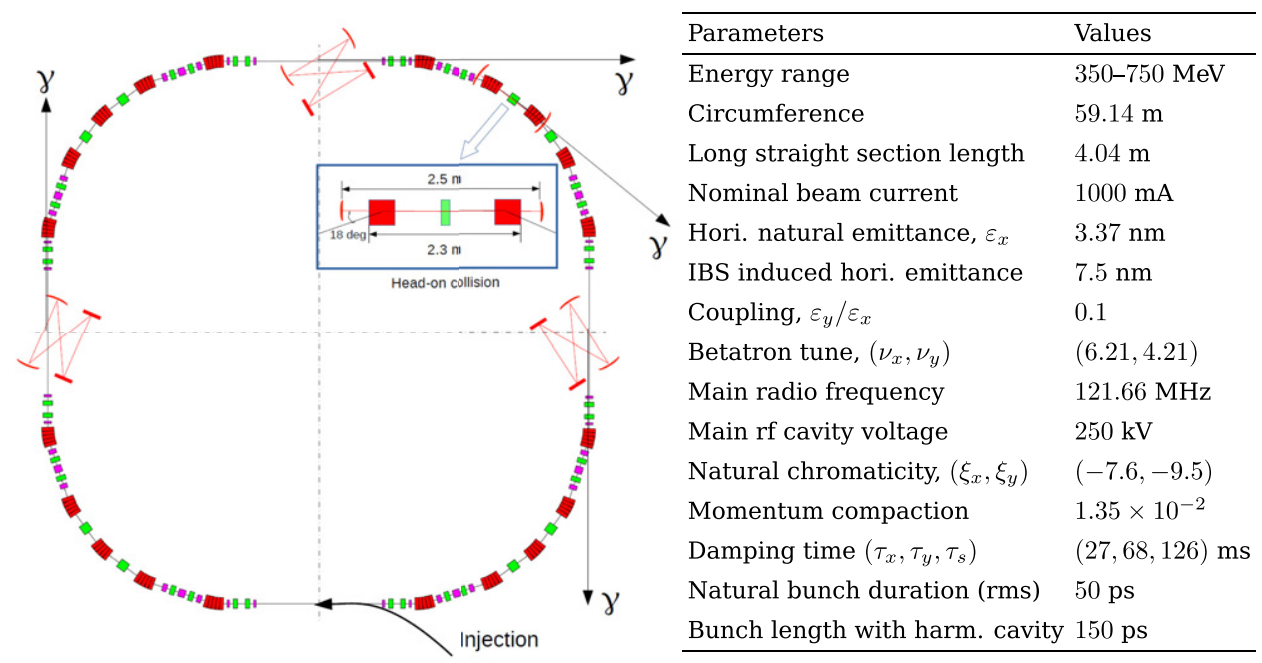

Figure 37. (Left) Layout and parameters of a low energy electron storage ring (350-750 MeV). Four CGSs are shown in two different collision configurations: one for the head-on collision using a two-mirror FP cavity in the arc and the other with a small crossing angle using a four-mirror cavity in the straight section. (Right) Main parameters for the storage ring, where all energy-dependent parameters are shown for $500 \mathrm{MeV}$ operation.

bremsstrahlung radiation, with two possible schemes illustrated in figure 35 . This, together with ultra-high vacuum realized in advanced storage rings [388], will reduce the radiation background by at least two orders of magnitude compared to the already very low background achieved at the HIGS facility.

The following is a prioritized list of important R \& D topics for such a medium-energy CGS:

\section{- High priority}

* To develop a dual-wavelength high-finesse FP-cavity for $1550 \mathrm{~nm}$ and $517 \mathrm{~nm}$;

* To design a reliable injector for single- and multi-bunch injection with high charge;

\section{- Medium priority}

* To design an energy-varying storage ring with small emittance and large dynamic aperture;

* To design a specialized interaction region with ultra-high vacuum either in an arc section or in a chicane to minimize radiation background;

* To develop laser beam optics for polarization switch (circular and linear), and for dual-wavelength operation;

* To design radiation shielding to handle high local electron loss.

5.3.2. Storage ring based low energy CGS. The required $\gamma$-ray beam performance at lower energies (table 12) can be realized with a storage ring based Compton CGS using a low energy electron beam (100s of $\mathrm{MeV}$ ) and multiple high power FP cavities. One possible design of such storage rings is shown in figure 37 with related machine design parameters listed. This $60 \mathrm{~m}$-long storage ring is comprised of four five-bend achromatic arcs with a small emittance and a reasonably large dynamic aperture. 


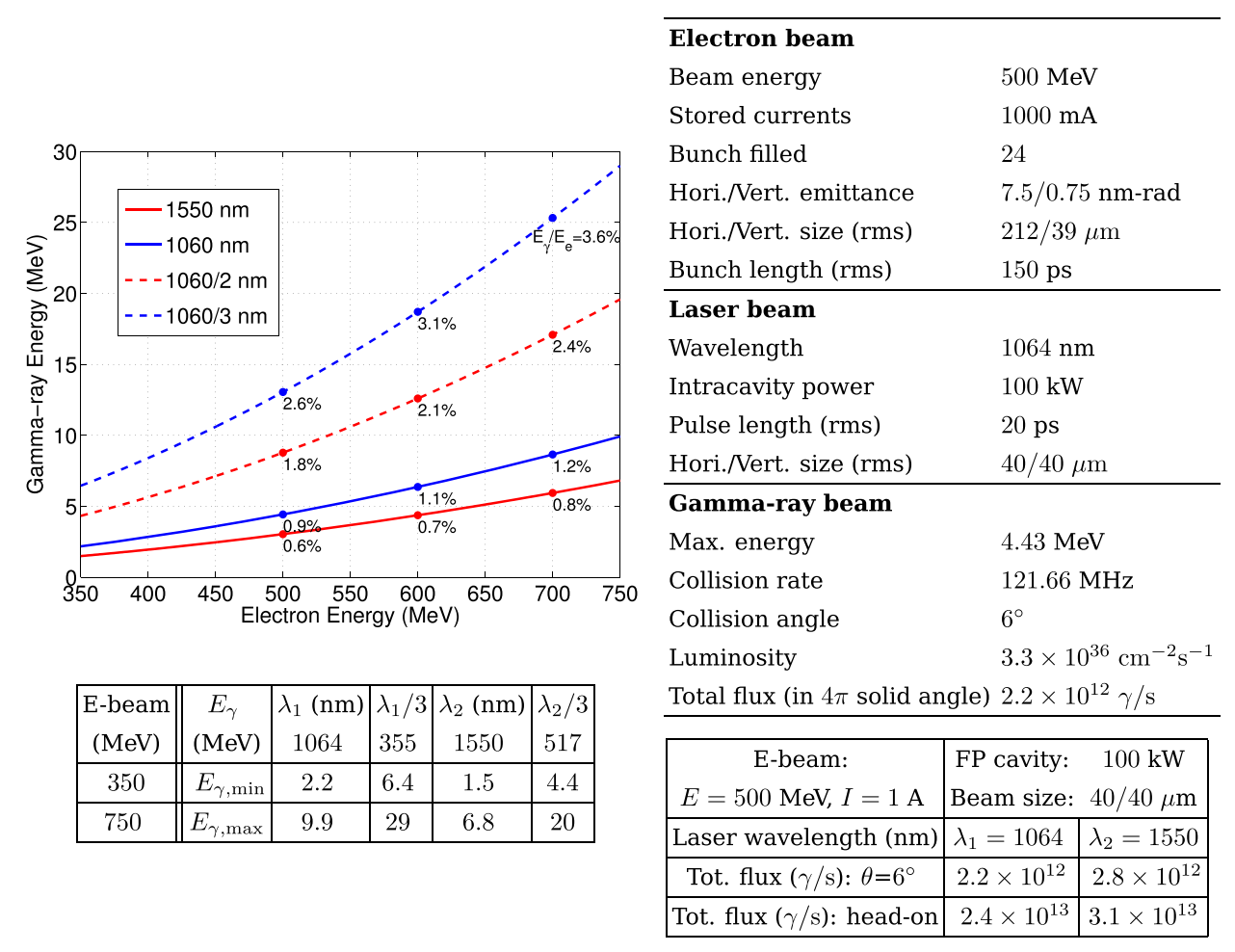

Figure 38. (Left) Energy of the $\gamma$-ray beam as a function of electron beam energy (350-750 MeV) for lasers at 1064 and $1550 \mathrm{~nm}$ and their third harmonics. (Upperright) A table listing the key operational parameters for producing a $4.4 \mathrm{MeV} \gamma$-ray beam using a $500 \mathrm{MeV}$ electron beam and a $1064 \mathrm{~nm}$ laser beam with a $6^{\circ}$ cross angle. (Lower-right) A table showing the total flux for $6^{\circ}$ cross-angle and head-on collision configurations with the same electron and laser beam parameters.

For this low energy storage ring design, the intrabeam scattering (IBS) effect needs to be properly controlled/mitigated. IBS is a process in which lossless collisions between electrons in the same bunch lead to an increase of the electron beam distribution in all three dimensions, which will result in the transverse beam size growth, especially for low energy and small-emittance storage rings. Because of a strong IBS effect, there is no need to push the low emittance limit. One such an example storage ring design is shown in figure 37 . At $500 \mathrm{MeV}$, this ring has a horizontal natural emittance about $3.4 \mathrm{~nm}$ rad and the IBS effect increases the beam emittance to about $7.5 \mathrm{~nm}$ rad with $1000 \mathrm{~mA}$ beam current in 24 bunches, and with the help of a third harmonic rf cavity. The third-order harmonic cavity is used to lengthen the electron bunch by a factor of three in order to increase the beam lifetime which is mainly limited by large angle electron-electron collisions (the so-called Touschek effect). A more complete design of a low energy storage ring based CGS with a modest intracavity power $(20 \mathrm{~kW})$ can be found in [389].

Energy range: this storage ring based Compton source is a versatile gamma-ray factory. It can produce $\gamma$ rays in a wide range of energies from 1.5 to about $30 \mathrm{MeV}$ (see the table in figure 38). This is done by changing the electron beam energy from 350 to $750 \mathrm{MeV}$, and by using FP cavities driven by commercial lasers at multiple wavelengths. 
Flux performance: at the low energy end, the $\gamma$-ray flux is determined by the electron beam current stored in the storage ring, intracavity laser power, and collision configuration. Below 5 to $8 \mathrm{MeV}$, very high flux performance is available using a high power FP cavity driven by lasers operating at their fundamental wavelengths (e.g. 1064 or $1550 \mathrm{~nm}$ ). For example, for production of $4.4 \mathrm{MeV} \gamma$ rays in a small cross-angle collision configuration $\left(\theta=6^{\circ}\right)$ using a $1000 \mathrm{~mA}, 500 \mathrm{MeV}$ electron beam and a $100 \mathrm{~kW}$ laser beam inside a $1064 \mathrm{~nm}$ FP cavity, the total $\gamma$-ray flux is estimated to be $2.2 \times 10^{12} \gamma / \mathrm{s}$, and the corresponding flux on target with $2 \%$ energy resolution (FWHM) is about $6.5 \times 10^{10} \gamma / \mathrm{s}$ (see a list of consistent operational parameters in the table in figure 38). Using the same electron and laser beam parameters, the total flux can be increased to $2-3 \times 10^{13} \gamma / \mathrm{s}$, assuming head-on collision can be realized. Between 15 and $30 \mathrm{MeV}$ using an FP cavity driven by either a 2nd or 3rd harmonic laser beam, the CGS will be operated in the electron-loss mode, producing a lower total flux, on the order of few $10^{11} \mathrm{\gamma} / \mathrm{s}$ as limited by the electron injection rate. Between 5 and $15 \mathrm{MeV}$, the total $\gamma$-ray flux will range from few $10^{11}$ to few $10^{12} \gamma / \mathrm{s}$ (or higher), determined by specific choices of operational parameters.

Electron beam: to achieve a high average current, the electron beam will be stored in multiple bunches, typically with a vacuum clearing gap. This gap in the $\gamma$-ray beam can be used as the start of the time trigger for many experiments. In the example storage ring (figure 37), when fully filled, the electron bunch rate is $122 \mathrm{MHz}$.

Laser beam: the FP cavities will be powered by commercial MHz lasers (fiber or solid state) with commonly available wavelengths around 1064 and $1550 \mathrm{~nm}$. The 2nd and/or 3rd harmonic laser beam can also be used. The lasers with high average power and excellent beam quality will be essential for achieving high flux.

FP cavity and collision scheme: both head-on collision and small cross-angle collision can be arranged. For the head-on collision, a simple and compact two-mirror cavity can be used; it may be possible to install such a cavity in an arc section between two adjacent dipole magnets as shown in figure 37. For the cross-angle collision, a four-mirror cavity can be implemented in a dedicated straight section.

Energy resolution: a beam energy resolution of about 2\% (FWHM) can be readily realized by properly collimating the $\gamma$-ray beam. To achieve higher resolution $(\leqslant 1 \%)$, a narrow-band drive laser will be required and the resolution impact due to the electron beam energy spread and angular spread should be minimized.

Beam polarization: highly polarized, well-collimated Compton $\gamma$-ray beams can be produced using a polarized drive laser beam. For certain experiments requiring helicity switch (as fast as $50 \mathrm{~Hz}$ ), polarizing optics (including Pockels cells) can be utilized outside the FP cavity.

Production modes: like a storage ring based synchrotron radiation user facility, this low energy gamma-ray factory can be operated as a multiple user facility with several $\gamma$-ray beamlines. As shown in figure 37, several Compton sources can be installed in straight sections and arc sections. For each source, a dedicated user target room with proper shielding and a beam dump will be constructed. The gamma-ray facility can be operated in the single- or multi-user mode. In the single-user mode, the experiment on the floor can take full control of the $\gamma$-ray beam production, including having the ability to change both electron beam current and energy. In the multi-user mode, all CGSs share the electron beam, therefore, individual beamlines do not have the control of the electron beam current and energy. Beamlines can be developed with the independent energy tuning capability - this can be realized by constructing a special FP cavity which allows variation of the collision angle. It is worth pointing out that the flux will be reduced as the collision angle is increased. This gamma-ray factory provides a full energy coverage (1.5-30 MeV) by operating multiple beamlines using lasers of several different wavelengths and by running the storage ring at a few pre-determined energies. 
Several challenges and important issues for this low energy source are summarized here:

- High power FP cavity design: the main challenge for this low energy source is to develop a very high power FP cavity ( $\geqslant 100 \mathrm{~kW}$ ) operating at 50 to $200 \mathrm{MHz}$ with a very small beam size at the collision point (radius $\sim 40 \mu \mathrm{m}$ or smaller). The smallest beam size practically realizable will be limited by the high intracavity power.

- Dual wavelength FP cavity: any CGS on this storage ring can greatly expand its energy coverage by using a dual-wavelength FP cavity (e.g. at both $1550 \mathrm{~nm}$ and $517 \mathrm{~nm}$ ).

- Electron injection rate: there are two viable choices for the injector: (1) a low-cost, fullenergy, top-off booster injector; and (2) a full-energy room-temperature linac injector at a higher cost. At low energies, the $\gamma$-ray flux is not limited by the injection rate. At higher energies in the electron-loss mode, the $\gamma$-ray flux will be limited by the electron injection rate.

The following is a prioritized list of important R \& D topics for such a low energy CGS:

\section{- High priority}

* To develop a very high power FP cavity $(\geqslant 100 \mathrm{~kW})$ with a small beam size $(\leqslant 40 \mu \mathrm{m})$

* To develop a dual-wavelength high-finesse FP-cavity (e.g. 1550/517 nm);

\section{- Medium priority}

* To design an energy-varying storage ring with small emittance and large dynamic aperture;

* To design a specialized interaction region with ultra-high vacuum either in an arc section or in a chicane to minimize radiation background;

* To develop laser beam optics for fast polarization switch (circular and linear), and for dual-wavelength operation;

* To design radiation shielding to handle large local electron loss.

5.3.3. Energy recovery linac based low-energy CGS. The required $\gamma$-ray beam performance at lower energies (table 12) can also be realized with a Compton CGS based on an ERL and one or more FP laser cavities. One possible design of such gamma source is shown in figure 39 with related machine parameters listed. This ERL is comprised of a double-sided linac with two-pass recirculation loop and an additional loop for $\gamma$-ray beam generation.

The key components of ERL are similar to the compact ERL [288], in which generation of a narrow-band Compton scattered beam was recently demonstrated. The main linac and the injector linac are both L-band $(1.3 \mathrm{GHz})$ superconducting structures. The electron gun is a high-voltage $(500 \mathrm{kV})$ DC gun with a multi-alkali photocathode, which can generate a small-emittance beam, $\varepsilon_{n} \leqslant 1 \mathrm{~mm}$ mrad, at a bunch charge of 200-300 pC [390].

Flux performance: the performance of an ERL with an electron beam energy of $500 \mathrm{MeV}$ and a laser wavelength of $1064 \mathrm{~nm}$ is shown in a table (see figure 40).

Energy range: a wide $\gamma$-ray energy range can be achieved by changing the electron energy and laser wavelength (1064 $\mathrm{nm}$ and $532 \mathrm{~nm}$ ). In this design, the electron beam energy is varied from 100 to $750 \mathrm{MeV}$ to cover $\gamma$-ray energies from 0.18 to $20 \mathrm{MeV}$. An electron beam below $370 \mathrm{MeV}$ can be delivered by bending the beam out after a single turn or single linac to simplify operation. 


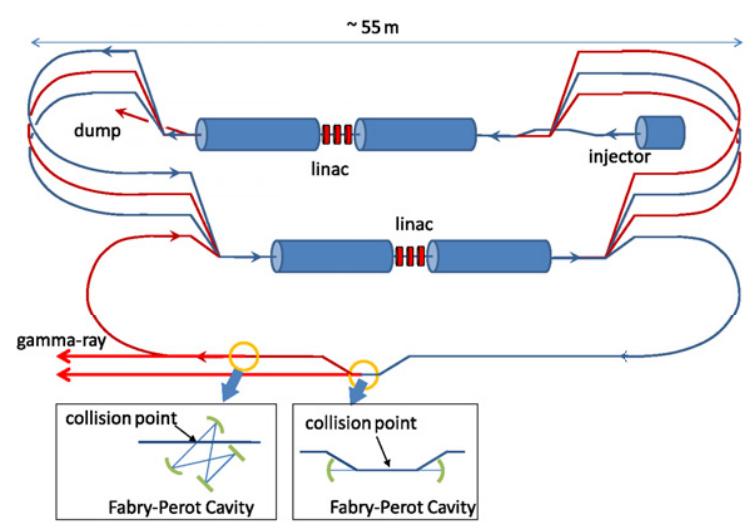

\begin{tabular}{ll}
\hline Parameters & Values \\
\hline Energy range & $100-750 \mathrm{MeV}$ \\
Bunch charge & $246 \mathrm{pC}$ \\
Bunch repetition & $81.25 \mathrm{MHz}$ \\
Ave. current & $20 \mathrm{~mA}$ \\
Norm. emittance $1 \mathrm{~mm}-\mathrm{mrad}$ \\
Bunch length & $10 \mathrm{ps}(\mathrm{rms})$ \\
Energy spread & $25 \mathrm{keV}(\mathrm{rms})$ \\
Collision spot & $15 \mu \mathrm{m}(\mathrm{rms})$ \\
\hline
\end{tabular}

Figure 39. (Left) Schematic layout and parameters of an ERL-based gamma source. The insets show two possible collision schemes. (Right) Electron beam parameters for the ERL.

Laser beam: because the energy range of the ERL is large, the laser system does not need to adjust to wavelengths longer than $1064 \mathrm{~nm}$. To get to higher $\gamma$-ray energies it will be necessary to frequency-double the laser light to $532 \mathrm{~nm}$ using an LBO crystal.

FP cavity and collision scheme: two types of FP cavities can be used for the $\gamma$-ray generation with a head-on or small cross-angle configuration as shown in figure 39. In order to stack laser pulses synchronizing with the electron bunch, the cavity frequency should be equal to the bunch repetition rate $(81.25 \mathrm{MHz})$ or its sub-harmonic. Parameters of laser beam and expected $\gamma$-ray flux are summarized in a table (part of figure 40 ). The flux requirement, $10^{13} \gamma / \mathrm{s}$, can be obtained with head-on collision, and an intra-cavity laser power of $70 \mathrm{~kW}$ with a tight laser focus at IP (beam size $\sim 15 \mu \mathrm{m}$ ). Separate cavities are needed for $1064 \mathrm{~nm}$ and $532 \mathrm{~nm}$ operation due to the need for very high reflectivity in the cavity.

Energy resolution: the ERL provides excellent beam quality so that the energy resolution is determined by the angular acceptance of the $\gamma$-ray beam collimation aperture. The high brightness of the electron beam allows a very small electron beam size even for a small beta function of $5-10 \mathrm{~cm}$ while still providing a small angular spread. The rms energy spread is typically much less than $10^{-3}$. The laser must be as narrow band as possible. A $\gamma$-ray beam energy spread of 1\%-2\% (FWHM) should be easily attainable.

Beam polarization: the $\gamma$-ray beam polarization is determined by the laser system. As with the storage ring design, polarization changes at a $50 \mathrm{~Hz}$ rate are attainable.

Production modes: an operation of low repetition rate, $10 \mathrm{MHz}$ or less, is possible by simply reducing electron bunch repetition or reducing the collision rate with a kicker magnet at the expense of total flux. Multiple collision points can be placed in the recirculation loop. Since the collision rate is small enough $\left(10^{-4}\right.$ for this design), electron beam degradation due to multiple collisions is negligible. As in the case of storage ring sources, simultaneous experiments will be possible at some fixed $\gamma$-ray energies unless a tunable laser or variable angle system is developed.

The proposed CGS can be realized with existing technologies in principle. Technical risks remaining to be resolved are summarized as follows:

Electron source performance: a photocathode used for the generation of small-emittance electron beams has a limited life time. The Cornell injector demonstrated extraction of a $40 \mathrm{~mA}$ beam for $4 \mathrm{~h}$ without significant reduction of quantum efficiency [391] and, using the same gun 


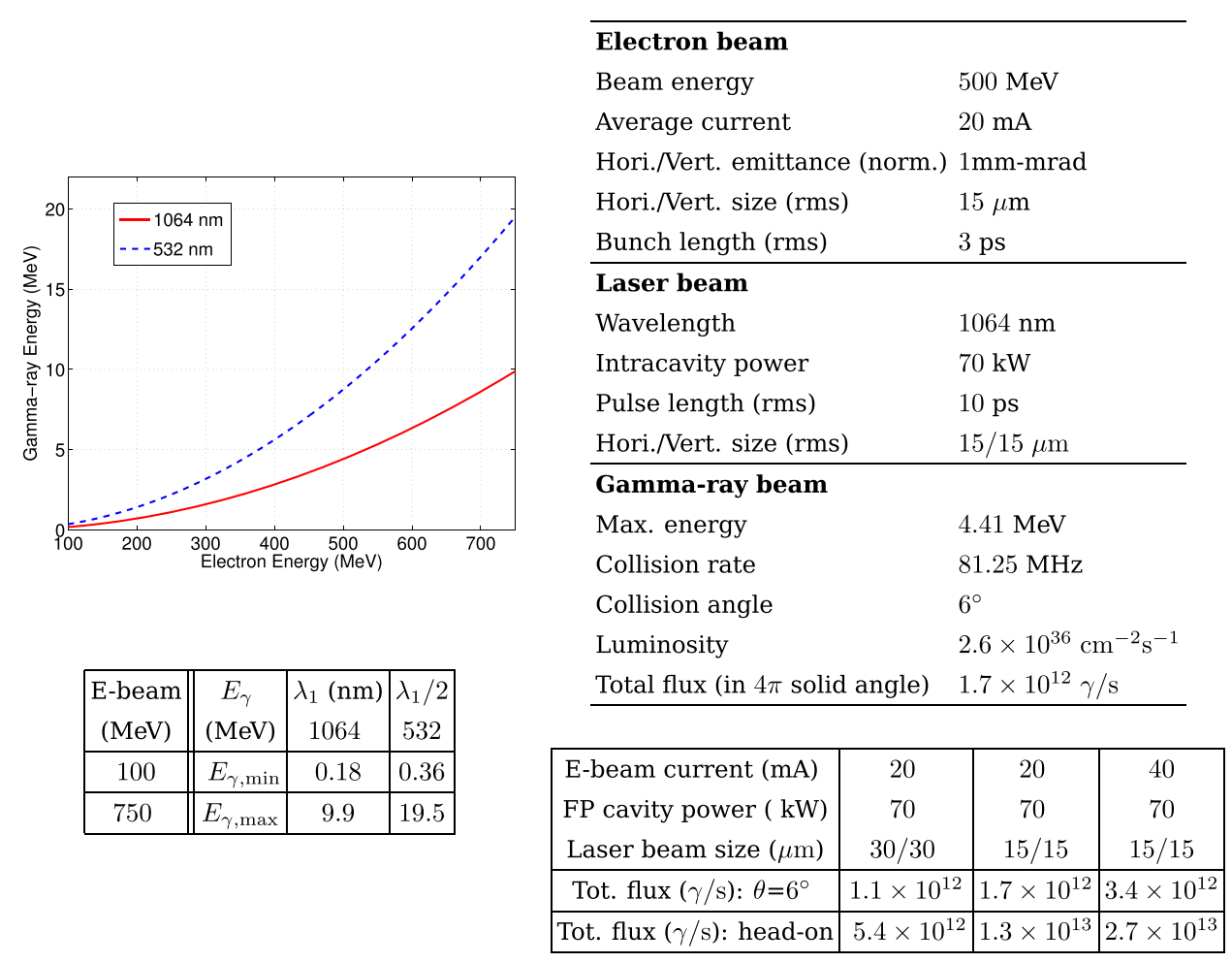

Figure 40. (Left) Energy of the $\gamma$-ray beam as a function of electron beam energy $(100-750 \mathrm{MeV})$ for lasers at 1064 and $532 \mathrm{~nm}$. (Upper-right) A table listing the key operational parameters for producing a $4.41 \mathrm{MeV} \gamma$-ray beam using a $500 \mathrm{MeV}$ electron beam and a $1064 \mathrm{~nm}$ laser beam with a $6^{\circ}$ cross angle. (Lower-right) A table showing the total flux for $6^{\circ}$ cross-angle and head-on collision configurations for two sets of values for the electron beam current and laser beam size at IP.

design, the LEReC project [345] at Brookhaven National Laboratory demonstrated multi-day operation at $30 \mathrm{~mA}$. The required emittance from the injector has been demonstrated [390]. Operation of an $8 \mathrm{~mA}, 115 \mathrm{MeV}$ ERL was demonstrated at JLab [392] and a proof-of-principle experiment of ERL-based Compton source was conducted at the compact ERL [288].

High current linac operation: the accelerator modules for this design will see up to $80 \mathrm{~mA}$ in total with accelerating and decelerating beams. This much current will produce copious higher order modes (HOMs) that must be heavily damped. L-band cryomodules for high-current beams have been designed and fabricated at Cornell and KEK [393, 394].

FP cavity performance: a FP cavity was developed at Lyncean Technologies to store laser power of $70 \mathrm{~kW}$ with a beam waist of $40 \mu \mathrm{m}$ [289] and a cavity at the cERL was $10 \mathrm{~kW}$ with $24 \mu \mathrm{m}$ (horizontal) and $32 \mu \mathrm{m}$ (vertical) spot sizes [288]. In addition, a cavity was developed for non-accelerator environment at Max-Planck Institute to store laser power of $670 \mathrm{~kW}$ with a reported $\mathrm{rms}$ beam size of $13 \mu \mathrm{m} \times 17 \mu \mathrm{m}$ at focus [369]. A FP cavity to store intra-cavity laser power of $70 \mathrm{~kW}$ with a beam waist of $15 \mu \mathrm{m}$ must be proven. Such a cavity requires state-of-the-art low-loss mirror coatings and thermally stable mirror substrates.

Preservation of injector beam quality: though the Cornell injector has demonstrated excellent performance, it is necessary to preserve this beam quality through a large number of long beam transport arcs. Each of these is subject to wakefields, longitudinal space charge 
(LSC) forces, and coherent synchrotron radiation (CSR) induced emittance growth. Designs have been developed to minimize any increase in the transverse emittance in an isochronous arc. The emittance growth can be kept to very small levels. A more serious problem is the longitudinal phase space distortion leading to a growth in the projected energy spread. Some of this may be compensated by rf cavities. A harmonic rf cavity might be required to take out higher-order curvature.

Operational issues: some of the scattered electrons may be lost when operating at very high photon energy. These losses must be controlled and understood. The levels of loss look tolerable and many electrons will still be energy-recovered but it is important to control where the electrons are lost. Continuous energy tuning in ERLs has not been demonstrated, yet. Sophisticated algorithms must be developed to tune up the beam transport at a new energy as the energy is changed.

We recommend to conduct the following $\mathrm{R} \& \mathrm{D}$ items:

\section{- High priority}

* Laser stacker at $1064 \mathrm{~nm}$ and $532 \mathrm{~nm}$ to verify the power, beam size, and intensity at the IP;

* Endurance runs with CsKSb cathodes at $20 \mathrm{~mA}$;

* Lattice designs for ERL with CGS with CSR and LSC compensation.

\section{- Medium priority}

* Beam breakup and HOM loading simulations for ERL with CGS (e.g. Cornell and KEK modules);

* Diagnostic development for high current beams and backscattering setup.

\section{Disclaimer}

This report was prepared as an account of work sponsored by an agency of the United States Government. Neither the United States Government nor any agency thereof, nor any of their employees, makes any warranty, express or implied, or assumes any legal liability or responsibility for the accuracy, completeness, or usefulness of any information, apparatus, product, or process disclosed, or represents that its use would not infringe privately owned rights. Reference herein to any specific commercial product, process, or service by trade name, trademark, manufacturer, or otherwise, does not necessarily constitute or imply its endorsement, recommendation, or favoring by the United States Government or any agency thereof. The views and opinions of authors expressed herein do not necessarily state or reflect those of the United States Government or any agency thereof. This workshop and the resulting whitepaper are supported by the US. Department of Energy Office of Nuclear Physics under Grant Number DE-SC0014616 and by the Triangle Universities Nuclear Laboratory (TUNL).

\section{Data availability statement}

All data that support the findings of this study are included within the article (and any supplementary files). 


\section{Appendix A}

\section{A.1. Workshop agenda}

Thursday, November 17, 2016|Overview Sessions, Grand Ballroom A

\begin{tabular}{|c|c|c|c|}
\hline Time & Title & Speaker & Affiliation \\
\hline $8: 45$ & Welcome and remarks & Calvin Howell & Duke U \& TUNL \\
\hline \multicolumn{4}{|c|}{ Compton gamma-ray sources } \\
\hline \multirow[t]{4}{*}{ 9:00 } & $\begin{array}{l}\text { (a) Global review of Compton } \gamma \text {-ray sources } \\
\text { (b) Facility talks }\end{array}$ & Ying Wu & Duke U \& TUNL \\
\hline & (b.1) HIGS and CGS projects in the US & Ying Wu & Duke U \& TUNL \\
\hline & (b.2) ELI-NP and CGS projects in Europe & Calin Ur & ELI-NP \\
\hline & (b.3) Compton $\gamma$-ray sources in Japan & Ryoichi Hajima & NIRS, Japan \\
\hline \multirow[t]{2}{*}{ 9:06 } & $\begin{array}{l}\text { (b.4) Compton } \gamma \text {-ray sources in China } \\
\text { (c) Possible technologies for } \\
\text { next-generation sources }\end{array}$ & Chuanxiang Tang & Tsinghua U, China \\
\hline & (c.1) High power lasers and optics & Fabian Zomer & LAL, France \\
\hline \multirow[t]{2}{*}{$9: 54$} & (c.2) Accelerator technologies & John Byrd & LBNL \\
\hline & (c.3) Compton source configuration strategies & Ying Wu & Duke U \& TUNL \\
\hline \multirow[t]{2}{*}{ 10:30 } & Break, Salons A \& B Foyer & & \\
\hline & \multicolumn{3}{|c|}{ Low-energy QCD } \\
\hline 10:50 & What to learn from nucleon polarizabilities & Harald Griesshammer & GWU \\
\hline 11:15 & Compton experiments I & Phil Martel & U Mainz \& Regina \\
\hline $11: 30$ & Compton experiments II & Gerald Feldman & GWU \\
\hline 11:45 & $\begin{array}{l}\text { Testing confinement scale QCD wWith } \\
\text { low-energy electromagnetic pion production }\end{array}$ & Aron Berstein & MIT \\
\hline $12: 35$ & Lunch, Salons A \& B Foyer & & \\
\hline 14:00 & Hadronic parity violation & W Michael Snow & Indiana $\mathrm{U}$ \\
\hline $14: 25$ & Nuclear structure & Deniz Savran & GSI, Germany \\
\hline \multirow[t]{2}{*}{ 14:55 } & Nuclear astrophysics & Carl Brune & Ohio U \\
\hline & \multicolumn{3}{|c|}{ Applications } \\
\hline $15: 20$ & Homeland security & Calvin Howell & Duke U \& TUNL \\
\hline $15: 35$ & National nuclear security & Matthew Durham & LANL \\
\hline 15:50 & Medical diagnosis & Anuj Kapadia & Duke U \\
\hline 16:05 & Charge to working groups & Calvin Howell & Duke U \& TUNL \\
\hline $16: 15$ & Break, Grand Ballroom Salons A \& B Foyer & & \\
\hline
\end{tabular}




Group

(1) Gamma-ray source

(2.a) LE QCD: nucleon polarizabilities

(2.b) LE QCD: photopion physics

(2.c) LE QCD: hadronic partity violation

(3) Nuclear structure and astrophysics

(4) Applications

Banquet
Location

Grand Ballroom B

Grand Ballroom A

Grand Ballroom A

Bethesda

Chevy Chase

Rockville

Congressional Ballroom

Friday, November 18, 2016|working group sessions

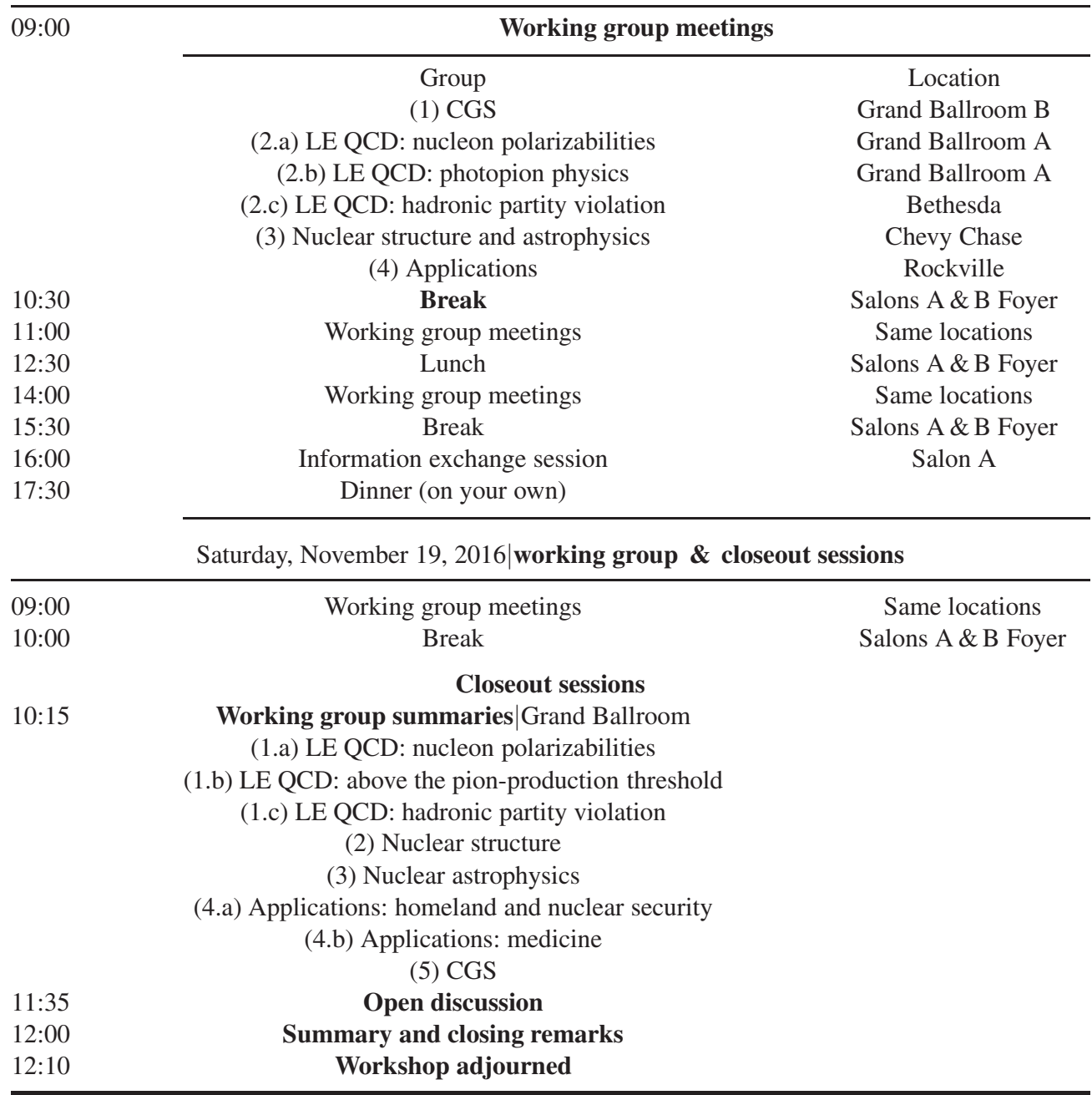




\section{A.2. Working group conveners}

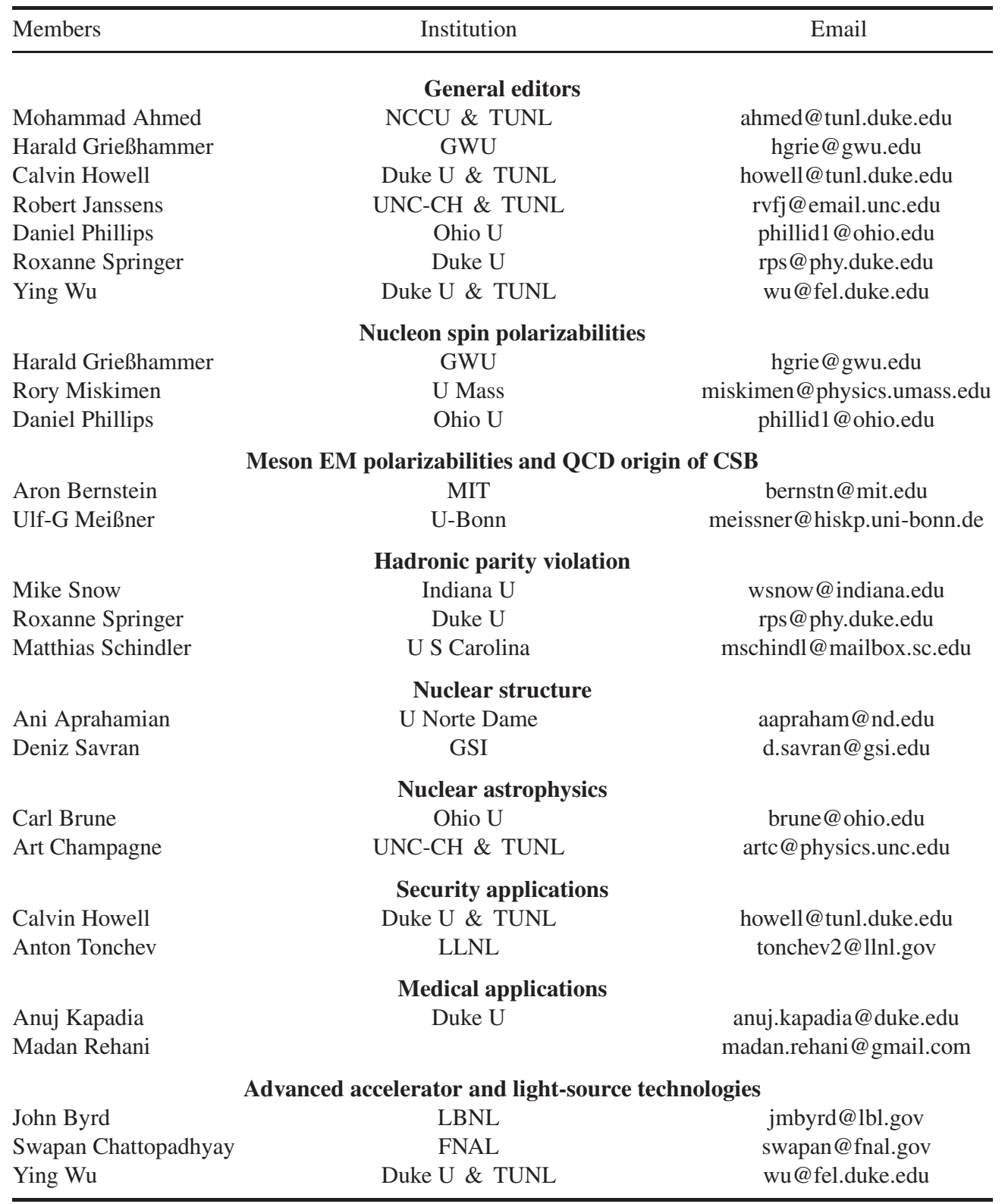




\section{A.3. Workshop participants}

\begin{tabular}{|c|c|c|c|}
\hline Last name & First name & Email & Working group \\
\hline Afanasev & Andrei & afanas@gwu.edu & Nuclear structure \\
\hline Ahmed & Mohammad & ahmed@tunl.duke.edu & QCD origin of CSB \\
\hline Alesini & David & david.alesini@lnf.infn.it & Accelerator \& light source \\
\hline Annand & John & john.annand@glasgow.ac.uk & Nucleon polarizabilities \\
\hline Aprahamian & Ani & aapraham@nd.edu & Nuclear structure \\
\hline Balabanski & Dimiter & dimiter.balabanski@eli-np.ro & Nuclear structure \\
\hline Barty & Christopher & barty1@1lnl.gov & Accelerator \& light source \\
\hline Benson & Stephen & felman@jlab.org & Accelerator \& light source \\
\hline Bernstein & Aron & bernstn@mit.edu & QCD origin of CSB \\
\hline Brune & Carl & brune@ohio.edu & Nuclear astrophysics \\
\hline Byrd & John & jmbyrd@lbl.gov & Accelerator \& light source \\
\hline Carlsten & Bruce & bcarlsten@lanl.gov & Accelerator \& light source \\
\hline Champagne & Art & artc@physics.unc.edu & Nuclear astrophysics \\
\hline Chattopadhyay & Swapan & swapan@fnal.gov & Accelerator \& light source \\
\hline Davis & David & eddaviddavis@gmail.com & HPV \\
\hline Downie & Evie & edownie@gwu.edu & Nucleon polarizabilities \\
\hline Durham & J Matthew & durham@lanl.gov & Applications \\
\hline Feldman & Gerald & feldman@gwu.edu & Nucleon polarizabilities \\
\hline Friesen & Forrest & fqf@phy.duke.edu & Nuclear structure \\
\hline Gao & Haiyan & gao@tunl.duke.edu & Nucleon polarizabilities \\
\hline Geddes & Cameron & cgrgeddes@lbl.gov & Accelerator \& light source \\
\hline Griesshammer & Harald & hgrie@gwu.edu & Nucleon polarizabilities \\
\hline Hajima & Ryoichi & hajima.ryoichi@qst.go.jp & Accelerator \& light source \\
\hline Haо & Hao & haohao@fel.duke.edu & Accelerator \& light source \\
\hline Holstein & Barry & holstein@physics.umass.edu & HPV \\
\hline Hornidge & David & dhornidg@mta.ca & Nucleon polarizabilities \\
\hline Howell & Calvin & howell@tunl.duke.edu & Applications \\
\hline Huffman & Paul & paul_huffman@ncsu.edu & HPV \\
\hline Iliadis & Christian & iliadis@physics.unc.edu & Nuclear astrophysics \\
\hline Isaak & Johann & jisaak@rcnp.osaka-u.ac.jp & Nuclear structure \\
\hline Kapadia & Anuj & anuj.kapadia@duke.edu & Applications \\
\hline Kendellen & David & dpkendel@tunl.duke.edu & Nucleon polarizabilities \\
\hline Krasznahorkay & Attila & kraszna@atomki.hu & Nuclear structure \\
\hline Kovash & Micheal & kovash@pa.uky.edu & Nucleon polarizabilities \\
\hline Lee & Dean & djlee3@unity.ncsu.edu & Nuclear structure \\
\hline Lorant & Csige & csige.lorant@atomki.mta.hu & Nuclear structure \\
\hline Martel & Phil & martel@kph.uni-mainz.de & Nucleon polarizabilities \\
\hline Meissner & Ulf & meissner@hiskp.uni-bonn.de & QCD origin of CSB \\
\hline Miskimen & Rory & miskimen@physics.umass.edu & Nucleon polarizabilities \\
\hline Moon & Namdoo & namdoo.moon@HQ.DHS.GOV & Government agency \\
\hline Mueller & Jonathan & jmmuell3@ncsu.edu & Applications \\
\hline
\end{tabular}




\begin{tabular}{|c|c|c|c|}
\hline Opper & Allena & aopper@nsf.gov & Government agency \\
\hline Pasquini & Barbara & pasquini@pv.infn.it & Nucleon polarizabilities \\
\hline Phillips & Daniel & phillid1@ohio.edu & Nucleon polarizabilities \\
\hline Pietralla & Norbert & pietralla@ikp.tu-darmstadt.de & Nuclear structure \\
\hline Rai & Gulshan & Gulshan.Rai@science.doe.gov & Government agency \\
\hline Rehani & Madan & madan.rehani@gmail.com & Applications \\
\hline Rhodes & William & William.Rhodes@nnsa.doe.gov & Government agency \\
\hline Savran & Deniz & d.savran@gsi.de & Accelerator \& light source \\
\hline Schindler & Matthias & MSCHINDL@mailbox.sc.edu & HPV \\
\hline Sikora & Mark & msikora@tunl.duke.edu & Nucleon polarizabilities \\
\hline Snow & Mike & wsnow@indiana.edu & HPV \\
\hline Springer & Roxanne & rps@phy.duke.edu & HPV \\
\hline Stone & Terri & Terri.Stone@nnsa.doe.gov & Government agency \\
\hline Sun & Changchun & ccsun@lbl.gov & Accelerator \& light source \\
\hline Tang & Chuanxiang & Tang.xuh@tsinghua.edu.cn & Accelerator \& light source \\
\hline Tiburzi & Brian & btiburzi@ccny.cuny.edu & Nucleon polarizabilities \\
\hline Tonchev & Anton & tonchev2@1lnl.gov & Applications \\
\hline Tornow & Werner & tornow@tunl.duke.edu & Nuclear structure \\
\hline Ur & Calin & calin.ur@eli-np.ro & Accelerator \& light source \\
\hline Wang & Dong & wangdong@sinap.ac.cn & Accelerator \& light source \\
\hline Weller & Henry & weller@tunl.duke.edu & QCD origin of CSB \\
\hline Werner & Volker & vw@ikp.tu-darmstadt.de & Nuclear structure \\
\hline $\mathrm{Wu}$ & Ying & wu@fel.duke.edu & Accelerator \& light source \\
\hline Yan & Jun & junyan@fel.duke.edu & Accelerator \& light source \\
\hline Zilges & Andreas & zilges@ikp.uni-koeln.de & Nuclear structure \\
\hline Zomer & Fabian & zomer@lal.in2p3.fr & Accelerator \& light source \\
\hline
\end{tabular}

\section{ORCID iDs}

M W Ahmed (1) https://orcid.org/0000-0002-3246-5027

A Afanasev (10 https://orcid.org/0000-0003-0679-3307

R Hajima (ㄴ) https://orcid.org/0000-0002-0383-9851

U-G Meißner (1) https://orcid.org/0000-0003-1254-442X

D R Phillips (1) https://orcid.org/0000-0003-1596-9087

W M Snow (1) https://orcid.org/0000-0002-8931-5513

B Tiburzi (이 https://orcid.org/0000-0001-8696-2902

\section{References}

[1] Pieper S C and Wiringa R B 2001 Annu. Rev. Nucl. Part. Sci. 5153

[2] Navrátil P et al 2007 Phys. Rev. Lett. 99042501

[3] Navratil P et al 2008 Few-Body Syst. 43129

[4] Nogga A, Navratil P, Barrett B R and Vary J P 2006 Phys. Rev. C 73064002

[5] Glöckle W et al 1996 Phys. Rep. 274107

[6] Machleidt R 2001 Phys. Rev. C 63024001

[7] Wiringa R B, Stoks V G J and Schiavilla R 1995 Phys. Rev. C 5138

[8] Stoks V G J, Klomp R A M, Terheggen C P F and de Swart J J 1994 Phys. Rev. C 492950

[9] Coon S A, Scadron M D and Barrett B R 1975 Nucl. Phys. A 242467

[10] Pieper S C, Pandharipande V R, Wiringa R B and Carlson J 2001 Phys. Rev. C 64014001

[11] Hammer H-W, König S and van Kolck U 2020 Rev. Mod. Phys. 92025004

[12] Epelbaum E, Krebs H and Reinert P 2020 Front. Phys. 898 
[13] Machleidt R and Entem D R 2011 Phys. Rep. 5031

[14] Epelbaum E, Hammer H-W and Meissner U-G 2009 Rev. Mod. Phys. 811773

[15] Beane S R, Chang E, Detmold W, Orginos K, Parreño A, Savage M J and Tiburzi B C 2015 Phys. Rev. Lett. 115132001

[16] Grießhammer H W, McGovern J A, Phillips D R and Feldman G 2012 Prog. Part. Nucl. Phys. 67 841

[17] Beane S R, Chang E, Cohen S, Detmold W, Lin H W, Orginos K, Parreño A, Savage M J and Tiburzi B C 2014 Phys. Rev. Lett. 113252001

[18] Howell C R, Tornow W and Witała H 2016 EPJ Web Conf. 11304008

[19] Savran D, Aumann T and Zilges A 2013 Prog. Part. Nucl. Phys. 70210

[20] Reinhard P-G and Nazarewicz W 2010 Phys. Rev. C 81051303

[21] Tamii A et al 2011 Phys. Rev. Lett. 107062502

[22] Piekarewicz J et al 2011 Phys. Rev. C 83034319

[23] Rossi D M et al 2013 Phys. Rev. Lett. 111242503

[24] Roca-Maza X et al 2015 Phys. Rev. C 92064304

[25] Baldo M and Burgio G F 2016 Prog. Part. Nucl. Phys. 91203

[26] Goriely S, Khan E and Samyn M 2004 Nucl. Phys. A 739331

[27] Litvinova E et al 2009 Phys. Rev. C 79054312

[28] Daoutidis I et al 2012 Phys. Rev. C 86034328

[29] Tonchev A et al 2017 Phys. Lett. B 77320

[30] Löher B et al 2013 Nucl. Instrum. Methods A 723136

[31] Löher B et al 2016 Phys. Lett. B 75672

[32] Copper N 2015 Doctoral Dissertation Yale University

[33] Isaak J 2016 Doctoral Dissertation Johannes Gutenberg-Universität Mainz

[34] Isaak J et al 2013 Pediatrics 131361

[35] Beck T et al 2017 Phys. Rev. Lett. 118212502

[36] Otsuka T, Suzuki T, Fujimoto R, Grawe H and Akaishi Y 2005 Phys. Rev. Lett. 95232502

[37] Otsuka T and Tsunoda Y 2016 J. Phys. G: Nucl. Part. Phys. 43024009

[38] Leoni S et al 2017 Phys. Rev. Lett. 118162502 Crider B P et al 2016 Phy. Lett. B 763108 Suchyta S et al 2014 Phys. Rev. C $89021301(\mathrm{R})$

[39] Pietralla N, von Brentano P and Lisetskiy A F 2008 Prog. Part. Nucl. Phys. 60225

[40] Beller J et al 2013 Phys. Rev. Lett. 111172501

[41] Goriely S 1998 Phys. Lett. B 43610

[42] Iachello F 1985 Phys. Lett. B 1601

[43] Savran D et al 2006 Phys. Rev. Lett. 97172502

[44] Endres J et al 2010 Phys. Rev. Lett. 105212503

[45] Savran D et al 2018 Phys. Lett. B 78616

[46] Krumbholz A et al 2015 Phys. Lett. B 7447

[47] Isaak J et al 2019 Phys. Lett. B 788225

[48] Krugmann A 2014 Doctoral Dissertation Technische Universität Darmstadt

[49] Butler P A and Nazarewicz W 1996 Rev. Mod. Phys. 68349

[50] Kneissel U, Pietralla N and Zilges A 2006 J. Phys. G: Nucl. Part. Phys. 32 R217

[51] Vonoertzen W, Freer M and Kanadaenyo Y 2006 Phys. Rep. 43243

[52] Astier A et al 2010 Phys. Rev. Lett. 104042701

[53] Röpke G et al 2014 Phys. Rev. C 90034304

[54] Pitz H H et al 1990 Nucl. Phys. A 509587

[55] Friedrichs H et al 1992 Phys. Rev. C 45 R892

[56] Eckert T et al 1997 Phys. Rev. C 561256

[57] Spieker M, Pascu S, Zilges A and Iachello F 2015 Phys. Rev. Lett. 114192504

[58] Ejiri H et al 2008 Eur. Phys. J. Spec. Top. 162239

[59] Ydrefors E, Balasi K G, Kosmas T S and Suhonen J 2012 Nucl. Phys. A 8781

[60] Thirolf P G and Habs D 2002 Prog. Part. Nucl. Phys. 49325

[61] Krasznahorkay A 2011 Handbook of Nuclear Chemistry (New York: Springer)

[62] McDonnell J, Nazarewicz W and Sheikh J 2013 Phys. Rev. C 87054327

[63] Jachimowicz P et al 2013 Phys. Rev. C 87044308

[64] Csige L et al 2009 Phys. Rev. C 80011301

[65] Berman B L and Fultz S C 1975 Rev. Mod. Phys. 47713 
[66] Vogt K et al 2001 Phys. Rev. C 63055802

[67] Sonnabend K, Vogt K, Galaviz D, Müller S and Zilges A 2004 Phys. Rev. C 70035802

[68] Erhard M et al 2006 Eur. Phys. J. A 27135

[69] Utsunomiya H et al 2003 Phys. Rev. C 67015807

[70] Sauter T and Käppeler F 1997 Phys. Rev. C 553127

[71] Bork J, Schatz H, Käppeler F and Rauscher T 1998 Phys. Rev. C 58524

[72] Özkan N et al 2002 Nucl. Phys. A 710469

[73] Rapp W et al 2002 Phys. Rev. C 66015803

[74] Gyürky G et al 2005 Nucl. Phys. A 758517

[75] Fülöp Z et al 2001 Phys. Rev. C 64065805

[76] Galaviz D et al 2005 Phys. Rev. C 71065802

[77] Lederer C et al 2013 Phys. Rev. Lett. 110022501

[78] Kratz K-L, Bitouzet J-P, Thielemann F-K, Moeller P and Pfeiffer B 1993 Astrophys. J. 403216

[79] Wallerstein G et al 1997 Rev. Mod. Phys. 69995

[80] Abbott B P et al (LIGO and Virgo Collaborations) 2017 Phys. Rev. Lett. 119161101

[81] Käppeler F 1999 Prog. Part. Nucl. Phys. 43419

[82] Käppeler F and Mengoni A 2006 Nucl. Phys. A 777291

[83] Dillmann I, Pardo C D, Käppeler F, Mengoni A and Sonnabend K 2008 Publ. Astron. Soc. Aust. 2518

[84] Takahashi K and Yokoi K 1983 Nucl. Phys. A 404578

[85] Bao Z Y, Beer H, Käppeler F, Voss F, Wisshak K and Rauscher T 2000 At. Data Nucl. Data Tables 7670

[86] Käppeler F, Gallino R, Bisterzo S and Aoki W 2011 Rev. Mod. Phys. 83157

[87] Lederer C et al 2014 Phys. Rev. C 89025810

[88] Rauscher T 2012 Astrophys. J. Lett. 755 L10

[89] Hauser W and Feshbach H 1952 Phys. Rev. 87366

[90] Tonchev A P et al 2010 Phys. Rev. Lett. 104072501

[91] Romig C et al 2013 Phys. Rev. C 88044331

[92] Fowler W A 1984 Rev. Mod. Phys. 56149

[93] deBoer R J et al 2017 Rev. Mod. Phys. 89035007

[94] Schürmann D et al 2005 Eur. Phys. J. A 26301

[95] Assunção M et al 2006 Phys. Rev. C 73055801

Fey M 2004 Doctoral Dissertation Universität Stuttgart, Germany

[96] Matei C et al 2006 Phys. Rev. Lett. 97242503

[97] Kunz R, Fey M, Jaeger M, Mayer A, Hammer J W, Staudt G, Harissopulos S and Paradellis T 2002 Astrophys. J. 567643

[98] Strieder F 2008 J. Phys. G: Nucl. Part. Phys. 35014009

[99] Gai M et al 2010 JINST 5 P12004

[100] Zimmerman W R et al 2013 Phys. Rev. Lett. 110152502

[101] DiGiovine B et al 2015 Nucl. Instrum. Methods Phys. Res. A 78196

[102] Haensel P, Potekhin A Y and Yakovlev D G 2007 Neutron Stars 1: Equation of State and Structure (New York: Springer)

[103] Lattimer J M and Prakash M 2000 Phys. Rep. 121333

[104] Henshaw S S et al 2011 Phys. Rev. Lett. 107222501

[105] Paar N, Vretenar D, Khan E and Colò G 2007 Rep. Prog. Phys. 70691

[106] Litvinova E, Ring P and Tselyaev V 2008 Phys. Rev. C 78014312

[107] Klimkiewicz A et al 2007 Phys. Rev. C 76 051603(R)

[108] Piekarewicz J 2006 Phys. Rev. C 73044325

[109] Krastev P G and Li B A 2010 arXiv:1001.0353

[110] Tamii A et al 2011 Phys. Rev. Lett. 107062502

[111] Wieland O et al 2009 Phys. Rev. Lett. 102092502

[112] Paar N et al 2014 Phys. Rev. C 90 011304(R)

[113] Beck D H and Holstein B R 2001 Int. J. Mod. Phys. E 101

[114] Beck D H and McKeown R D 2001 Annu. Rev. Nucl. Part. Sci. 51189

[115] Beck D H 2003 Prog. Part. Nucl. Phys. 50429

[116] Beise E J, Pitt M L and Spayde D T 2005 Prog. Part. Nucl. Phys. 54289

[117] Adelberger E G and Haxion W C 1985 Annu. Rev. Nucl. Part. Sci. 35501

[118] Bowman J D, Garvey G T, Johnson M B and Mitchell G E 1993 Annu. Rev. Nucl. Part. Sci. 43829 
[119] Tomsovic S, Johnson M B, Hayes A and Bowman J D 2000 Phys. Rev. C 62054607

[120] Zeldovich Y B 1957 Sov. Phys. - JETP 21184

[121] Flambaum V V and Khriplovich I B 1980 Sov. Phys. - JETP 52835

[122] Wood C S et al 1997 Science 2751759

[123] Tsigutkin K et al 2009 Phys. Rev. Lett. 103071601

[124] Bowman J D and Gudkov V 2014 Phys. Rev. C 90065503

[125] Wasem J 2012 Phys. Rev. C 85 022501(R)

[126] Holstein B, Haxton W and Savage M 2016 Frontiers in Nuclear Physics, KITP Workshop (22 August-4 November)

[127] Danilov G S 1965 Phys. Lett. 1840

[128] Zhu S-L, Maekawa C M, Holstein B R, Ramsey-Musolf M J and van Kolck U 2005 Nucl. Phys. A $\mathbf{7 4 8} 435$

[129] Girlanda L 2008 Phys. Rev. C 77067001

[130] de Vries J, Meißner U-G, Epelbaum E and Kaiser N 2013 Eur. Phys. J. A 49149

[131] Viviani M, Baroni A, Girlanda L, Kievsky A, Marcucci L E and Schiavilla R 2014 Phys. Rev. C 89064004

[132] de Vries J, Li N, Meißner U-G, Kaiser N, Liu X-H and Zhu S-L 2014 Eur. Phys. J. A 50108

[133] de Vries J, Li N, Meißner U-G, Nogga A, Epelbaum E and Kaiser N 2015 Phys. Lett. B 747299

[134] Phillips D R, Samart D and Schat C 2015 Phys. Rev. Lett. 114062301

[135] Schindler M R, Springer R P and Vanasse J 2016 Phys. Rev. C 93025502

[136] Blyth D et al 2018 Phys. Rev. Lett. 121242002

[137] Snow W M et al 2015 Rev. Sci. Instrum. 86055101

[138] Walker-Loud A, Berkowitz E, Haxton W, Kurth T, Nicholson A and Wasem J 2016 Incite Award, First Lattice QCD Calculation of the I $=2$ Two-Nucleon Parity Violating Amplitude

[139] Kurth T, Walker-Loud A, Nicholson A, Berkowitz E, Vranas P, Strother M and Rinaldi E 2016 PoS LATTICE2015 329

[140] Earle E D, McDonald A B, Kidner S H, Clifford E T H, Hill J J, Keech G H, Chupp T E and Schneider M B 1988 Can. J. Phys. 66534

[141] Knyaz'kov V A et al 1984 Nucl. Phys. A 417209

[142] Sinclair C et al 2000 Letter-of-Intent 00-002 for PAC 17: Study of the Parity Nonconserving Force between Nucleons through Deuteron Photodisintegration

[143] Wojtsekhowski B and van Oers W T H 2000 Summary of the Working Group Meeting on Parity Violation in Deuteron Photodisintegration with Circularly Polarized Photons (13-14 April) (Jefferson Lab)

[144] Schindler M R and Springer R P 2010 Nucl. Phys. A 84651

[145] Vanasse J and Schindler M R 2014 Phys. Rev. C 90044001

[146] Desplanques B, Donoghue J F and Holstein B R 1980 Ann. Phys. 124449

[147] Schiavilla R, Carlson J and Paris M 2004 Phys. Rev. C 70044007

[148] Bowman J 2007 INT program 'fundamental neutron physics' http://int.washington.edu/talks/ WorkShops/int_07_1/

[149] Titov A I, Fujiwara M and Kawase K 2006 J. Phys. G: Nucl. Part. Phys. 321097

[150] Bertozzi W and Ledoux R J 2005 Nucl. Instrum. Methods Phys. Res. B 241820

[151] Pruet J, McNabb D P, Hagmann C A, Hartemann F V and Barty C P J 2006 J. Appl. Phys. 99 123102

[152] Hagmann C A, Hall J M, Johnson M S, McNabb D P, Kelley J H, Huibregtse C, Kwan E, Rusev $\mathrm{G}$ and Tonchev A P 2009 J. Appl. Phys. 106084901

[153] Kolokytha S et al 2017 Adv. Sci. Technol. Eng. Syst. J. 260

[154] Hagelstein F, Miskimen R and Pascalutsa V 2016 Prog. Part. Nucl. Phys. 8829

[155] Wissmann F 2004 Compton Scattering: Investigating the Structure of the Nucleon with Real Photons (Springer Tracts in Modern Physics vol 200) (Cham: Springer)

[156] Schumacher M 2005 Prog. Part. Nucl. Phys. 55567

[157] Drechsel D, Pasquini B and Vanderhaeghen M 2003 Phys. Rep. 37899

[158] Pasquini B and Vanderhaeghen M 2018 Annu. Rev. Nucl. Part. Sci. 6875

[159] Gasser J, Hoferichter M, Leutwyler H and Rusetsky A 2015 Eur. Phys. J. C 75375

[160] Walker-Loud A, Carlson C E and Miller G A 2012 Phys. Rev. Lett. 108232301

[161] Pohl R, Gilman R, Miller G A and Pachucki K 2013 Annu. Rev. Nucl. Part. Sci. 63175

[162] Birse M C and McGovern J A 2012 Eur. Phys. J. A 48120 
[163] Aprahamian A et al 2015 Reaching for the Horizon: The 2015 Long Range Plan for Nuclear Science

[164] Committee on the Assessment of and Outlook for Nuclear Physics, National Research Council of the National Academies 2012 Nuclear Physics: Exploring the Heart of Matter (Washington, DC, USA)

[165] European Science Foundation 2017 NuPECC Long Range Plan 2017-Perspectives in Nuclear Physics

[166] Martel P (The Mainz A2 Collaboration) 2015 Phys. Rev. Lett. 114112501

[167] Paudyal D et al (A2 Collaboration) 2019 arXiv:1909.02032 [nucl-ex]

[168] Lujan M, Alexandru A, Freeman W and Lee F X 2014 Phys. Rev. D 89074506

[169] Freeman W, Alexandru A, Lujan M and Lee F X 2014 Phys. Rev. D 90054507

[170] Lujan M, Alexandru A, Freeman W and Lee F X 2016 Phys. Rev. D 94074506

[171] Primer T, Kamleh W, Leinweber D and Burkardt M 2014 Phys. Rev. D 89034508

[172] Beane S R et al 2014 Phys. Rev. Lett. 113252001

[173] Beane S R, Chang E, Detmold W, Orginos K, Parreño A, Savage M J and Tiburzi B C (NPLQCD Collaboration) 2015 Phys. Rev. Lett. 115132001

[174] Chang E et al 2015 Phys. Rev. D 92114502

[175] Detmold W, Orginos K, Parreño A, Savage M J, Tiburzi B C, Beane S R and Chang E 2016 Phys. Rev. Lett. 116112301

[176] Detmold W, Edwards R G, Dudek J J, Engelhardt M, Lin H-W, Meinel S, Orginos K and Shanahan P (USQCD Collaboration) 2019 Eur. Phys. J. A 55193

[177] Engelhardt M 2011 arXiv:1111.3686v1 [hep-lat]

[178] Griesshammer H W, McGovern J A and Phillips D R 2016 Eur. Phys. J. A 52139

[179] Bernstein A 2016 private communication

[180] Griesshammer H W, L'vov A I, McGovern J A, Pascalutsa V, Pasquini B and Phillips D R 2014 Compton Scattering off Protons and Light Nuclei (Trento, Italy 29 July-2 August 2013)

[181] Babusci D, Giordano G, L'vov A I, Matone G and Nathan A M 1998 Phys. Rev. C 581013

[182] Grießhammer H W, McGovern J A and Phillips D R 2018 Eur. Phys. J. A 5437

[183] Pasquini B, Drechsel D and Vanderhaeghen M 2007 Phys. Rev. C 76015203

[184] Grießhammer H W 2013 Eur. Phys. J. A 49100

Grießhammer H W 2017 Eur. Phys. J. A 53113 (erratum)

Grießhammer H W 2018 Eur. Phys. J. A 5457

[185] Margaryan A, Strandberg B, Griesshammer H W, Mcgovern J A, Phillips D R and Shukla D 2018 Eur. Phys. J. A 54125

[186] Demissie B T 2018 Inelastic Compton scattering on the deuteron and extraction of neutron polarizabilities $P h D$ Thesis George Washington University

[187] Olmos de León V et al 2001 Eur. Phys. J. A 10207

[188] Levchuk M I and L'vov A I 2000 Nucl. Phys. A 674449

[189] Gryniuk O, Hagelstein F and Pascalutsa V 2015 Phys. Rev. D 92074031

[190] McGovern J A, Phillips D R and Grießhammer H W 2013 Eur. Phys. J. A 4912

[191] Pasquini B, Pedroni P and Sconfietti S 2018 Phys. Rev. C 98015204

[192] Krupina N, Lensky V and Pascalutsa V 2018 Phys. Lett. B 78234

[193] Pasquini B, Pedroni P and Sconfietti S 2019 J. Phys. G: Nucl. Part. Phys. 46104001

[194] Myers L S et al 2014 Phys. Rev. Lett. 113262506

[195] Myers L S et al 2015 Phys. Rev. C 92025203

[196] Sokhoyan V et al (A2 Collaboration) 2017 Eur. Phys. J. A 5314

[197] Martel P, Biroth M, Collicott C, Paudyal D and Rajabi A (A2 Collaboration) 2017 EPJ Web Conf. 14201021

[198] Collicott C 2015 PhD Thesis Dalhousie University

[199] Kramer K et al 2007 Nucl. Instrum. Methods A 582318

[200] Ye Q, Laskaris G, Chen W, Gao H, Zheng W, Zong X, Averett T, Cates G D and Tobias W A 2010 Eur. Phys. J. A 4455

[201] Zhao Z 2020 private communication Duke University

[202] Sikora M H et al 2017 Phys. Rev. C 96055209

[203] Wright D H, Debevec P T, Morford L J and Nathan A M 1985 Phys. Rev. C 321174

[204] Feldman G et al 2016 Proposal for HIGS Experiment E-08-16: Compton Scattering from ${ }^{3} \mathrm{He}$

[205] Kossert K et al 2002 Phys. Rev. Lett. 88162301 
[206] Kendellen D P, Ahmed M W, Baird E, Feldman G, Perreau N, Wallace P W and Weller H R 2016 Nucl. Instrum. Methods Phys. Res. A 840174

[207] Thomas A 2011 Eur. Phys. J. Spec. Top. 198171

[208] Biroth M, Achenbach P, Downie E and Thomas A 2016 PoS PSTP2015 005

[209] Biroth M, Achenbach P, Downie E and Thomas A 2015 Nucl. Instrum. Methods Phys. Res. A 787 68

[210] Biroth M personal communication

[211] Chen J-P et al 2016 Jlab Polarized ${ }^{3}$ He Target System (Virginia, USA: Newport News)

[212] Kramer K et al 2007 Nucl. Instrum. Methods A 582318

[213] Laskaris G et al 2015 Phys. Lett. B 750547

[214] Patrignani C et al 2016 Chin. Phys. C 40100001

[215] Bernard V, Kaiser N and Meißner U-G 1996 Chiral corrections to the Kroll-Rudermann theorem Phys. Lett. B 383116

[216] Meißner U-G 1995 private communication

[217] Gibbs W R, Ai L and Kaufmann W B 1995 Phys. Rev. Lett. 743740

[218] Bernstein A M 1993 Infect. Dis. Newsl. 1255

[219] Weinberg S 1966 Phys. Rev. Lett. 17616

[220] Compton A H 1923 A quantum theory of the scattering of x-rays by light elements Phys. Rev. 21 483

[221] Compton A H 1922 Secondary radiations produced by x-rays Bull. Natl Res. Counc. 420

[222] Milburn R H 1963 Electron scattering by an intense polarized photon field Phys. Rev. Lett. 1075

[223] Arutyunian F R and Tumanian V A 1963 The Compton effect on relativistic electrons and the possibility of obtaining high energy beams Phys. Lett. 4176

[224] Kulikov O F, Telnov Y Y, Filippov E I and Yakimenko M N 1964 Compton effect on moving electrons Phys. Lett. 13344

[225] Bemporad C et al 1965 High-energy photons from Compton scattering of light on $6.0 \mathrm{GeV}$ electrons Phys. Rev. B 1381546

[226] Murray J J and Klein P R 1967 A Compton scattered laser beam for the 82 bubble chamber SLAC Report No. SLAC-TN-67-19 (unpublished)

[227] Ballam J et al 1969 Total and partial photoproduction cross sections at 1.44, 2.8, and $4.7 \mathrm{GeV}$ Phys. Rev. Lett. 23498

[228] Casano L et al 1975 Frascati report LNF-74/60 (1974) Laser and Unconv. Opt. J. 553

[229] Matone G et al 1977 Lect. Notes Phys. 623

[230] Federici L et al 1980 The LADON photon beam with the ESRF $5 \mathrm{GeV}$ machine Lett. Nuovo Cimento 27339

[231] Federici L et al 1980 Backward Compton scattering of laser light against high-energy electrons: the LADON photon beam at Frascati Nuovo Cimento B 59247

[232] Babusci D et al 1991 Taladon: a polarized and tagged gamma ray beam Nucl. Instrum. Methods Phys. Res. A 30519

[233] Sandorfi A M, LeVine M J, Thorn C E, Giordano G, Matone G and Schaerf C 1983 High energy gamma ray beams from Compton backscattered laser light IEEE Trans. Nucl. Sci. 303083

[234] Litvinenko V N et al 1997 Gamma-ray production in a storage ring free-electron laser Phys. Rev. Lett. 784569

[235] Babusci D, Casano L, D’Angelo A, Picozza P, Schaerf C and Girolami B 1990 Project GRAAL: the scientific case Nuovo Cimento A 1031555

[236] Kazakov A A et al 1984 Fisson of the ${ }^{238} \mathrm{U}$ and ${ }^{237} \mathrm{~Np}$ nuclei by $\gamma$-quanta of intermediate energies Physics and Mathematics (JPRS-UPM-86-001), p 24 (SEE N86-26108 16-70)

Kazakov A A et al 1984 Pisma V Zhurnal Eksperimentalnoy I Teoreticheskoy Fiziki (Moscow, USSR) 40 445-7 (Engl. transl.)

[237] Kezerashvili G Y, Milov A M and Wojtsekhowski B B 1993 The gamma ray energy tagging spectrometer of the ROKK-2 facility at the VEPP-3 storage ring Nucl. Instrum. Methods Phys. Res. A 328506

[238] Kezerashvili G Y et al 1995 ROKK-1M is the Compton source of the high intensity polarized and tagged gamma beam at the VEPP-4M collider AIP Conf. Proc. 343260

[239] Kezerashvili G Y, Milov A M, Muchnoi N Y and Usov A P 1998 A Compton source of high energy polarized tagged $\gamma$-ray beams. The ROKK-1M facility Nucl. Instrum. Methods Phys. Res. B 145 40 
[240] Nakano T et al 1998 New projects at SPring-8 with multi-GeV polarized photons Nucl. Phys. A $629559 \mathrm{c}$

[241] Nakano T et al 2001 Multi-GeV laser-electron photon project at SPring-8 Nucl. Phys. A $68471 \mathrm{c}$

[242] Yamazaki T et al 1998 SRFEL, FEL-X project, and applications at the ETL Nucl. Instrum. Methods Phys. Res. B 14483

[243] Hosaka M, Hama H, Kimura K, Yamazaki J and Kinoshita T 1997 Observation of intracavity Compton backscattering of the UVSOR free electron laser Nucl. Instrum. Methods Phys. Res. A 393525

[244] Taira Y, Adachi M, Zen H, Tanikawa T, Yamamoto N, Hosaka M, Takashima Y, Soda K and Katoh M 2011 Generation of energy-tunable and ultra-short-pulse gamma rays via inverse Compton scattering in an electron storage ring Nucl. Instrum. Methods Phys. Res. A 652696

[245] Zen $\mathrm{H}$ et al 2016 Generation of high energy gamma-ray by laser Compton scattering of $1.94 \mu \mathrm{m}$ fiber laser in UVSOR-III electron storage ring Energy Proc. 89335

[246] Sei N, Ogawa H and Yamada K 2011 Lasing of middle-infrared free-election lasers using the storage ring NIJI-IV Opt. Lett. 363645

[247] Ogawa H, Sei N and Yamada K 2012 Asymmetric two-bunch operation of free-electron laser and generation of inverse Compton photons Phys. Lett. A 3761171

[248] Kaneyasu T, Takabayashi Y, Iwasaki Y and Koda S 2011 Generation of laser Compton gamma-rays in the SAGA light source storage ring Nucl. Instrum. Methods Phys. Res. A $\mathbf{6 5 9} 30$

[249] Horikawa K, Miyamoto S, Amano S and Mochizuki T 2010 Measurements for the energy and flux of laser Compton scattering $\gamma$-ray photons generated in an electron storage ring: NewSUBARU Nucl. Instrum. Methods Phys. Res. A 618209

[250] Nutarelli D, Coupriea M E, Nahon L, Bakker R, Delboulbe A, Roux R, Visentin B and Billardon M 1998 Gamma rays production by intra-cavity Compton back scattering with super-ACO storage ring free electron laser Nucl. Instrum. Methods A $\mathbf{4 0 7} 459$

[251] De Ninno G, Nutarelli D, Garzella D, Nahon L, Hirsch M, Renault E, Visentin B and Couprie M E 2001 Gamma rays produced by inverse Compton scattering in the super-ACO storage ring free electron laser Radiat. Phys. Chem. 61351

[252] Barty C P J 2015 Advanced laser-Compton gamma-ray sources for nuclear materials detection, assay and imaging Nuclear Physics and Gamma-Ray Sources for Nuclear Security and Nonproliferation: Proc. of the Int. Symp. (World Scientific) p 85

[253] Albert F et al 2010 Isotope-specific detection of low-density materials with laser-based monoenergetic gamma-rays Opt. Lett. 35354

[254] Albert F et al 2011 Design of narrow-band Compton scattering sources for nuclear resonance fluorescence Phys. Rev. ST Accel. Beams 14050703

[255] Albert F, Anderson S G, Gibson D J, Marsh R A, Siders C W, Barty C P J and Hartemann F V 2011 Three-dimensional theory of weakly nonlinear Compton scattering Phys. Plasmas 18013108

[256] Gibson D J et al 2010 Design and operation of a tunable MeV-level Compton-scattering-based $\gamma$-ray source Phys. Rev. ST Accel. Beams 13070703

[257] Barty C P J et al 2011 Overview of MEGa-ray-based nuclear materials management activities at the Lawrence Livermore National Laboratory Proc. of the 52nd Annual Meeting of the Institute for Nuclear Materials Management vol 3p 2645

[258] Hall J et al 2011 Numerical simulation of nuclear materials detection, imaging and assay with MEGa-rays Proc. of the 52nd Annual Meeting of the Institute for Nuclear Materials Management vol 3 p 2673

[259] Anderson S G et al 2011 VELOCIRAPTOR: an X-band photoinjector and linear accelerator for the production of mono-energetic Nucl. Instrum. Methods Phys. Res. A 657140

[260] Albert F et al 2012 Precision linac and laser technologies for nuclear photonics gamma-ray sources Phys. Plasmas 19056701

[261] Babusci D et al 1996 Polarised and tagged gamma-ray ladon beams Riv. Nuovo Cimento 191

[262] D'Angelo A et al 2000 Generation of Compton backscattering $\gamma$-ray beams Nucl. Instrum. Methods A 4551

[263] Schaerf C 2005 Polarized gamma-ray beams Phys. Today 5844

[264] Weller H R, Ahmed M W, Gao H, Tornow W, Wu Y K, Gai M and Miskimen R 2009 Research opportunities at the upgraded HI $\gamma$ S facility Prog. Part. Nucl. Phys. 62257

[265] Krafft G A and Priebe G 2010 Compton sources of electromagnetic radiation Rev. Accel. Sci. Technol. 03147 
[266] Litvinenko V N et al 1998 First UV/visible lasing with the OK-4/Duke storage ring FEL Nucl. Instrum. Methods A 4078

[267] Litvinenko V N et al 1999 OK-4/Duke storage ring FEL lasing in the deep-UV Nucl. Instrum. Methods Phys. Res. A 429151

[268] Litvinenko V N, Park S H, Pinayev I V and Wu Y 2001 Performance of the OK-4/Duke storage ring FEL Nucl. Instrum. Methods Phys. Res. A 47066

[269] Wu Y K, Vinokurov N A, Mikhailov S, Li J and Popov V 2006 High-gain lasing and polarization switch with a distributed optical-klystron free-electron laser Phys. Rev. Lett. 96224801

[270] Jia B et al 2010 Electron beam energy spread measurements using optical klystron radiation Phys. Rev. ST Accel. Beams 13080702

[271] Sun C et al 2009 Energy and energy spread measurements of an electron beam by Compton scattering method Phys. Rev. ST Accel. Beams 12062801

[272] Sun C, Wu Y K, Rusev G and Tonchev A P 2009 End-to-end spectrum reconstruction method for analyzing Compton gamma-ray beams Nucl. Instrum. Methods Phys. Res. A 605312

[273] Sun C and Wu Y K 2011 Theoretical and simulation studies of characteristics of a Compton light source Phys. Rev. ST Accel. Beams 14044701

[274] Weller H R, Ahmed M W and Wu Y K 2015 Nuclear physics research at the high intensity gammaray source $(\mathrm{HI} \gamma \mathrm{S})$ Nucl. Phys. News 2519

[275] Muramatsu N et al 2014 Development of high intensity laser-electron photon beams up to $2.9 \mathrm{GeV}$ at the SPring-8 LEPS beamline Nucl. Instrum. Methods Phys. Res. A 737184

[276] Luo W et al 2011 A 4D Monte Carlo laser-Compton scattering simulation code for the characterization of the future energy-tunable SLEGS Nucl. Instrum. Methods Phys. Res. A 660 108

[277] Xu H, Fan G, Wu H, Chen J, Xu B, Xu W and Wang D 2016 Interaction chamber design for an energy continuously tunable sub-MeV laser-Compton gamma-ray source IEEE Trans. Nucl. Sci. 63906

[278] Chen H et al 2019 Commissioning the photoinjector of a gamma-ray light source Phys. Rev. Accel. Beams 22053403

[279] Barletta W A and Borland M 2010 Report of the basic energy sciences workshop on compact light sources Technical Report United StatesUSDOE Office of Science (SC)

[280] Leemans W et al 1995 Femtosecond x-ray generation through $90^{\circ}$ Thomson scattering: status of the LBL experiment AIP Conf. Proc. 335209

[281] Carroll F E, Mendenhall M H, Traeger R H, Brau C and Waters J W 2003 Pulsed tunable monochromatic x-ray beams from a compact source:new opportunities Am. J. Roentgenol. 181 1197

[282] Du Y et al 2013 Generation of first hard x-ray pulse at tsinghua thomson scattering x-ray source Rev. Sci. Instrum. 84053301

[283] Zhang H 2017 Experimental polarization control of thomson scattering x-ray source 13th Symp. on Accelerator Physics p 176

[284] Chaleil A et al 2016 Inverse Compton scattering x-ray source yield optimization with a laser path folding system inserted in a pre-existent RF linac Nucl. Instrum. Methods Phys. Res. A 840113

[285] Glotin F, Ortega J-M, Prazeres R, Devanz G and Marcouillé O 1996 Tunable x-ray generation in a free-electron laser by intracavity Compton backscattering Phys. Rev. Lett. 773130

[286] Boyce J R et al 2003 The Jefferson lab sub-picosecond x-ray program AIP Conf. Proc. 680325

[287] Shimizu H et al 2015 X-ray generation by inverse Compton scattering at the superconducting RF test facility Nucl. Instrum. Methods Phys. Res. A 77226

[288] Akagi T et al 2016 Narrow-band photon beam via laser Compton scattering in an energy recovery linac Phys. Rev. Accel. Beams 19114701

[289] Eggl E, Dierolf M, Achterhold K, Jud C, Günther B, Braig E, Gleich B and Pfeiffer F 2016 The munich compact light source: initial performance measures J. Synchrotron Radiat. 231137

[290] Variola A et al 2014 ThomX technical design report Technical Report, HAL Id: In2p3-00971281

[291] Variola A et al 2014 The ThomX project status 5th Int. Particle Accel. Conf. IPAC'14Joint Accelerator Conferences Website p 2062

[292] Leemans W and Esarey E 2009 Laser-driven plasma-wave electron accelerators Phys. Today 62 44

[293] Esarey E, Schroeder C B and Leemans W P 2009 Physics of laser-driven plasma-based electron accelerators Rev. Mod. Phys. 811229 
[294] Schwoerer H et al 2006 Thomson-backscattered x rays from laser-accelerated electrons Phys. Rev. Lett. 96014802

[295] Ta Phuoc K, Corde S, Thaury C, Malka V, Tafzi A, Goddet J P, Shah R C, Sebban S and Rousse A 2012 All-optical Compton gamma-ray source Nat. Photon. 6308

[296] Chen S et al $2013 \mathrm{MeV}$-energy x rays from inverse Compton scattering with laser-wakefield accelerated electrons Phys. Rev. Lett. 110155003

[297] Powers N D, Ghebregziabher I, Golovin G, Liu C, Chen S, Banerjee S, Zhang J and Umstadter D P 2014 Quasi-monoenergetic and tunable x-rays from a laser-driven Compton light source Nat. Photon. 828

[298] Sarri G et al 2014 Ultrahigh brilliance multi-MeV $\gamma$-ray beams from nonlinear relativistic thomson scattering Phys. Rev. Lett. 113224801

[299] Rykovanov S G, Geddes C G R, Vay J-L, Schroeder C B, Esarey E and Leemans W P 2014 Quasi-monoenergetic femtosecond photon sources from Thomson scattering using laser plasma accelerators and plasma channels J. Phys. B: At. Mol. Opt. Phys. 47234013

[300] Khrennikov K, Wenz J, Buck A, Xu J, Heigoldt M, Veisz L and Karsch S 2015 Tunable all-optical quasimonochromatic thomson x-ray source in the nonlinear regime Phys. Rev. Lett. 114195003

[301] Yan W et al 2017 High-order multiphoton Thomson scattering Nat. Photon. 11514

[302] Cole J M et al 2018 Experimental evidence of radiation reaction in the collision of a high-intensity laser pulse with a laser-wakefield accelerated electron beam Phys. Rev. X 8011020

[303] Hooker S M 2013 Developments in laser-driven plasma accelerators Nat. Photon. 7775

[304] Corde S, Ta Phuoc K, Lambert G, Fitour R, Malka V, Rousse A, Beck A and Lefebvre E 2013 Femtosecond x rays from laser-plasma accelerators Rev. Mod. Phys. 851

[305] Albert F and Thomas A G R 2016 Applications of laser wakefield accelerator-based light sources Plasma Phys. Control. Fusion 58103001

[306] Martz H E, Glenn S M, Smith J A, Divin C J and Azevedo S G 2017 Poly- versus mono-energetic dual-spectrum non-intrusive inspection of Cargo containers IEEE Trans. Nucl. Sci. 641709

[307] Geddes C et al 2017 Impact of monoenergetic photon sources on nonproliferation applications final report Technical Report Idaho Falls, ID, United StatesIdaho National Lab. (INL)

[308] Krasny M W 2015 The gamma factory proposal for CERN (arXiv:1511.07794)

[309] Krasny M W et al 2018 The CERN Gamma Factory initiative: an ultra-high intensity gamma source Proc. IPAC vol 2018 p 1780

[310] Schaumann M et al 2019 First partially stripped ions in the LHC $\left({ }^{208} \mathrm{~Pb}^{81+}\right)$ Proc., 10th Int. Particle Accelerator Conf. (IPAC2019) (Melbourne, Australia May 19-24 2019) p MOPRB055

[311] Gamma Factory 2019 Proof-of-Principle Experiment, Letter of Intent, CERN-SPSC-2019031/SPSC-I-253 2019 http://cds.cern.ch/record/2690736/files/SPSC-I-253.pdf (accessed 25 September 2019)

[312] Selph F B et al 1988 The LBL 1-2 GeV synchrotron radiation source Nucl. Instrum. Methods Phys. Res. A 26644

[313] Galayda J N 1995 The advanced photon source Proc. of the 1995 Particle Accel. Conf. vol 1 (IEEE) $\mathrm{p} 4$

[314] Laclare J L 1993 Commissioning and performance of the ESRF Proc. 15th Particle Accel. Conf. (PAC'93) (IEEE) p 1427

[315] Zhao Z T et al 2009 Commissioning the Shanghai Light Source Proc. of PAC09 p 55

[316] Namkung W 2010 Review of third generation light sources Proc. of IPAC'10 p 2411

[317] Martensson N and Eriksson M 2018 The saga of MAX IV, the first multi-bend achromat synchrotron light source Nucl. Instrum. Methods Phys. Res. A 90797

[318] Steier C et al 2018 Status of the conceptual design of ALS-U Proc. 9th Int. Particle Accel. Conf. (IPAC'18) p 4134

[319] Henderson S 2015 Status of the APS upgrade project Proc. 6th Int. Particle Accel. Conf. (IPAC'15) p 1791

[320] Raimondi P 2016 The ESRF low-emittance upgrade Proc. 7th Int. Particle Accel. Conf. (IPAC'16) p 2023

[321] Hettel R 2014 Challenges in the design of diffraction-limited storage rings Proc. 5th Int. Particle Accel. Conf. (IPAC'14) p 7

[322] Borland M, Decker G, Emery L, Sajaev V, Sun Y and Xiao A 2014 Lattice design challenges for fourth-generation storage-ring light sources J. Synchrotron Radiat. 21912 
[323] Byrd J M, Santis S D, Georgsson M, Stover G, Fox J D and Teytelman D 2000 Commissioning of a higher harmonic RF system for the advanced light source Nucl. Instrum. Methods Phys. Res. A 455271

[324] Teytelman D et al 2011 Overview of system specifications for bunch-by-bunch feedback systems Proc. of PAC11 p 1475

[325] Wu W Z et al 2011 Development of a bunch-by-bunch longitudinal feedback system with a wide dynamic range for the HIGS facility Nucl. Instrum. Methods Phys. Res. A 63232

[326] Autin B and Marti Y 1973 Closed orbit correction of AG machines using a small number of magnets Technical Report (CERN)

[327] Chung Y, Decker G and Evans K 1993 Closed orbit correction using singular value decomposition of the response matrix Proc. of the IEEE Particle Accel. Conf. vol 3 (IEEE) p 2263

[328] Endo K, Fukuma H and Zhang F Q 1996 Preliminary orbit measurement for beam-based alignment Technical Report KEK Preprint 96-56

[329] Carwardine J A and Lenkszus F R 1998 Real-time orbit feedback at the APS AIP Conf. Proc. 451 125

[330] Boge M et al 1999 Fast closed orbit control in the SLS storage ring Proc. of the IEEE Particle Accel. Conf. vol 2 (IEEE) p 1129

[331] Schilcher T et al 2004 Commissioning and operation of the SLS fast orbit feedback EPAC vol $4 \mathrm{p}$ 2523

[332] Uzun I S et al 2005 Initial design of the fast orbit feedback system for diamond light source Proc. of ICALEPCS

[333] Röjsel P 1994 A beam position measurement system using quadrupole magnets magnetic centra as the position reference Nucl. Instrum. Methods Phys. Res. A 343374

[334] Portmann G, Robin D and Schachinger L 1995 Automated beam based alignment of the ALS quadrupoles Proc. Particle Accel. Conf. vol 4 (IEEE) p 2693

[335] Safranek J 1997 Experimental determination of storage ring optics using orbit response measurements Nucl. Instrum. Methods Phys. Res. A 38827

[336] Safranek J 2002 MATLAB-BASED LOCO Technical Report (SLAC National Accelerator Laboratory (SLAC))

[337] Knott M et al 1993 EPICS: a control system software co-development success story Technical Report (IL, United States: Argonne National Lab.)

[338] Dalesio L R, Hill J O, Kraimer M, Lewis S, Murray D, Hunt S, Watson W, Clausen M and Dalesio J 1994 The experimental physics and industrial control system architecture: past, present, and future Nucl. Instrum. Methods Phys. Res. A 352179

[339] Borland M and Emery L 1995 The self-describing data sets file protocol and Toolkit Technical Report (IL, United States: Argonne National Lab.)

[340] Borland M 1995 Self-describing file protocol for simulation integration and shared postprocessors Proc. of the IEEE Particle Accel. Conf. vol 4 (IEEE) p 2184

[341] Corbett J, Portmann G and Terebilo A 2003 Accelerator control middle layer Proc. of the IEEE Particle Accel. Conf. vol 4 p 2369

[342] Portmann G, Corbett J and Terebilo A 2005 An accelerator control middle layer using Matlab Proc. of the IEEE Particle Accel. Conf. vol 2005 p 4009

[343] Wu Y K, Hartman S and Mikhailov S F 2003 A physics based control system for the Duke storage ring Proc. of the IEEE Particle Accel. Conf. vol 4 p 2482

[344] Raubenheimer T 2016 LCLS-II-HE FEL facility overview Workshop on Scientific Opportunities for Ultrafast Hard X-Rays at High Rep.

[345] Kayran D et al 2018 LEReC photocathode DC gun beam test results Proc. 9th Int. Particle Accel. Conf. (IPAC'18), Int. Particle Accel. Conf. (Vancouver, BC, Canada 29 April-4 May 2018) (Geneva, Switzerland: JACoW Publishing) p 1306

[346] Miyajima T 2019 Compact ERL (cERL), stable $1 \mathrm{~mA}$ operation with a small beam emittance at KEK 63rd ICFA Advanced Beam Dynamics Workshop on Energy Recovery Linacs

[347] Pudasaini U et al 2019 Initial growth of tin on niobium for vapor diffusion coating of $\mathrm{Nb}_{3} \mathrm{Sn}$ Supercond. Sci. Technol. 32045008

[348] Williams P H et al 2018 A staged, multi-user x-ray free electron laser \& nuclear physics facility based on a multi-pass recirculating superconducting CW linac Presented at the 60th ICFA Advanced Beam Dynamics Workshop on Future Light Sources (FLS'18)

[349] Gulliford C et al 2019 CBETA beam commissioning results 10th Int. Particle Accel. Conf. (IPAC'19) (Geneva, Switzerland: JACOW Publishing) p 748 
[350] Rollason A J, Fang X and Dugdale D E 2004 Multipass optical cavity for inverse Compton interactions Nucl. Instrum. Methods Phys. Res. A 526560

[351] Shverdin M Y et al 2010 High-power picosecond laser pulse recirculation Opt. Lett. 352224

[352] Dupraz K et al 2014 Design and optimization of a highly efficient optical multipass system for $\gamma$ ray beam production from electron laser beam Compton scattering Phys. Rev. ST Accel. Beams 17033501

[353] Kogelnik H and Li T 1966 Laser beams and resonators Appl. Opt. 51550

[354] Arnaud J A 1970 Nonorthogonal optical waveguides and resonators Bell Syst. Tech. J. 492311

[355] Habraken S J M and Nienhuis G 2007 Modes of a twisted optical cavity Phys. Rev. A 75033819

[356] Zomer F, Fedala Y, Pavloff N, Soskov V and Variola A 2009 Polarization induced instabilities in external four-mirror Fabry-Perot cavities Appl. Opt. 486651

[357] Saraf S, Byer R L and King P J 2007 High-extinction-ratio resonant cavity polarizer for quantumoptics measurements Appl. Opt. 463850

[358] Liu X et al 2016 Laser frequency stabilization using folded cavity and mirror reflectivity tuning Opt. Commun. 36984

[359] Ozawa A, Zhao Z, Kuwata-Gonokami M and Kobayashi Y 2015 High average power coherent vuv generation at $10 \mathrm{MHz}$ repetition frequency by intracavity high harmonic generation $\mathrm{Opt}$. Express 2315107

[360] Sakaue K et al 2011 Development of a laser pulse storage technique in an optical super-cavity for a compact X-ray source based on laser-Compton scattering Nucl. Instrum. Methods Phys. Res. A $637 \mathrm{~S} 107$

[361] Drever R W P, Hall J L, Kowalski F V, Hough J, Ford G M, Munley A J and Ward H 1983 Laser phase and frequency stabilization using an optical resonator Appl. Phys. B 3197

[362] Udem T, Holzwarth R and Hänsch T W 2002 Optical frequency metrology Nature 416233

[363] Jason Jones R, Diels J-C, Jasapara J and Rudolph W 2000 Stabilization of the frequency, phase, and repetition rate of an ultra-short pulse train to a Fabry-Perot reference cavity Opt. Commun. 175409

[364] Jones R J and Diels J-C 2001 Stabilization of femtosecond lasers for optical frequency metrology and direct optical to radio frequency synthesis Phys. Rev. Lett. 863288

[365] Jones R J, Thomann I and Ye J 2004 Precision stabilization of femtosecond lasers to high-finesse optical cavities Phys. Rev. A 69051803

[366] Falletto N et al 2001 Compton scattering off polarized electrons with a high-finesse Fabry-Pérot cavity at JLab Nucl. Instrum. Methods Phys. Res. A 459412

[367] Baudrand S et al 2010 A high precision Fabry-Perot cavity polarimeter at HERA J. Inst. 5 P06005

[368] Honda Y et al 2005 Upgraded laser wire beam profile monitor Nucl. Instrum. Methods Phys. Res. A 538100

[369] Carstens H et al 2014 Megawatt-scale average-power ultrashort pulses in an enhancement cavity Opt. Lett. 392595

[370] Börzsönyi A et al 2013 External cavity enhancement of picosecond pulses with 28000 cavity finesse Appl. Opt. 528376

[371] Bonis J et al 2012 Non-planar four-mirror optical cavity for high intensity gamma ray flux production by pulsed laser beam Compton scattering off GeV-electrons J. Inst. 7 P01017

[372] Chaikovska I et al 2016 High flux circularly polarized gamma beam factory: coupling a Fabry-Perot optical cavity with an electron storage ring Sci. Rep. 636569

[373] Fukuda M et al 2015 Generation of multi-bunch beam with beam loading compensation by using rf amplitude modulation in laser undulator compact x-ray (LUCX) IPAC Proc.

[374] Shimizu H, Aryshev A, Higashi Y, Honda Y and Urakawa J 2014 Development of a four-mirror optical cavity for an inverse Compton scattering experiment in the STF Nucl. Instrum. Methods Phys. Res. A 74563

[375] Skettrup T 2005 Rectangular laser resonators with astigmatic compensation J. Opt. A: Pure Appl. Opt. 7645

[376] Klemz G, Mönig K and Will I 2006 Design study of an optical cavity for a future photon collider at ILC Nucl. Instrum. Methods Phys. Res. A 564212

[377] Putnam W P, Schimpf D N, Abram G and Kärtner F X 2012 Bessel-Gauss beam enhancement cavities for high-intensity applications Opt. Express 2024429

[378] Carstens H, Holzberger S, Kaster J, Weitenberg J, Pervak V, Apolonski A, Fill E, Krausz F and Pupeza I 2013 Large-mode enhancement cavities Opt. Express 2111606 
[379] Dupraz K, Cassou K, Martens A and Zomer F 2015 The ABCD matrix for parabolic reflectors and its application to astigmatism free four-mirror cavities Opt. Commun. 353178

[380] Wu Y K, Yan J, Hao H, Li J Y, Mikhailov S F, Popov V G, Vinokurov N A, Huang S and Wu J 2015 Widely tunable two-color free-electron laser on a storage ring Phys. Rev. Lett. 115184801

[381] Yan J et al 2016 Storage ring two-color free-electron laser Phys. Rev. Accel. Beams 19070701

[382] Yan J et al 2019 Precision control of gamma-ray polarization using a crossed helical undulator free-electron laser Nat. Photon. 13629

[383] Wu Y K 2015 Light source and accelerator physics research program at Duke University Proc. of International Particle Conf. (Richmond, VA, USA)

[384] Adriani O et al 2014 EuroGammaS proposal for the ELI-NP gamma beam system (arXiv:1407.3669)

[385] Alesini D et al 2015 DA $\Phi$ NE gamma-rays factory IEEE Trans. Nucl. Sci. 63913

[386] Tarawneh H, Eriksson M, Lindgren L-J and Anderberg B 2003 MAX-IV lattice, dynamic properties and magnet system Nucl. Instrum. Methods Phys. Res. A $\mathbf{5 0 8} 480$

[387] Farvacque L et al 2013 A low-emittance lattice for the ESRF Proc. of Int. Particle Conf. (Shanghai, China)

[388] Eriksson M et al 2016 Commissioning of the max IV light source Proc. of Int. Particle Conf. (Busan, Korea)

[389] Pan Z et al 2019 Design and dynamic studies for a compact storage ring to generate gamma-ray light source based on Compton backscattering technique Phys. Rev. Accel. Beams 22040702

[390] Gulliford C, Bartnik A, Bazarov I, Dunham B and Cultrera L 2015 Demonstration of cathode emittance dominated high bunch charge beams in a DC gun-based photoinjector Appl. Phys. Lett. 106094101

[391] Dunham B et al 2013 Record high-average current from a high-brightness photoinjector Appl. Phys. Lett. 102034105

[392] Neil G R et al 2006 The JLab high power ERL light source Nucl. Instrum. Methods Phys. Res. A 5579

[393] Eichhorn R et al 2016 First cool-down of the Cornell ERL main linac cryo-module Proc. IPAC vol 2016 p 2305

[394] Umemori K et al 2014 Operation status of compact ERL main linac cryomodule Proc. IPAC vol 2014 p 2537 